\title{
Results from Accelerated Environmental Testing of Barcoded Identifiers for UF 6 Cylinders
}

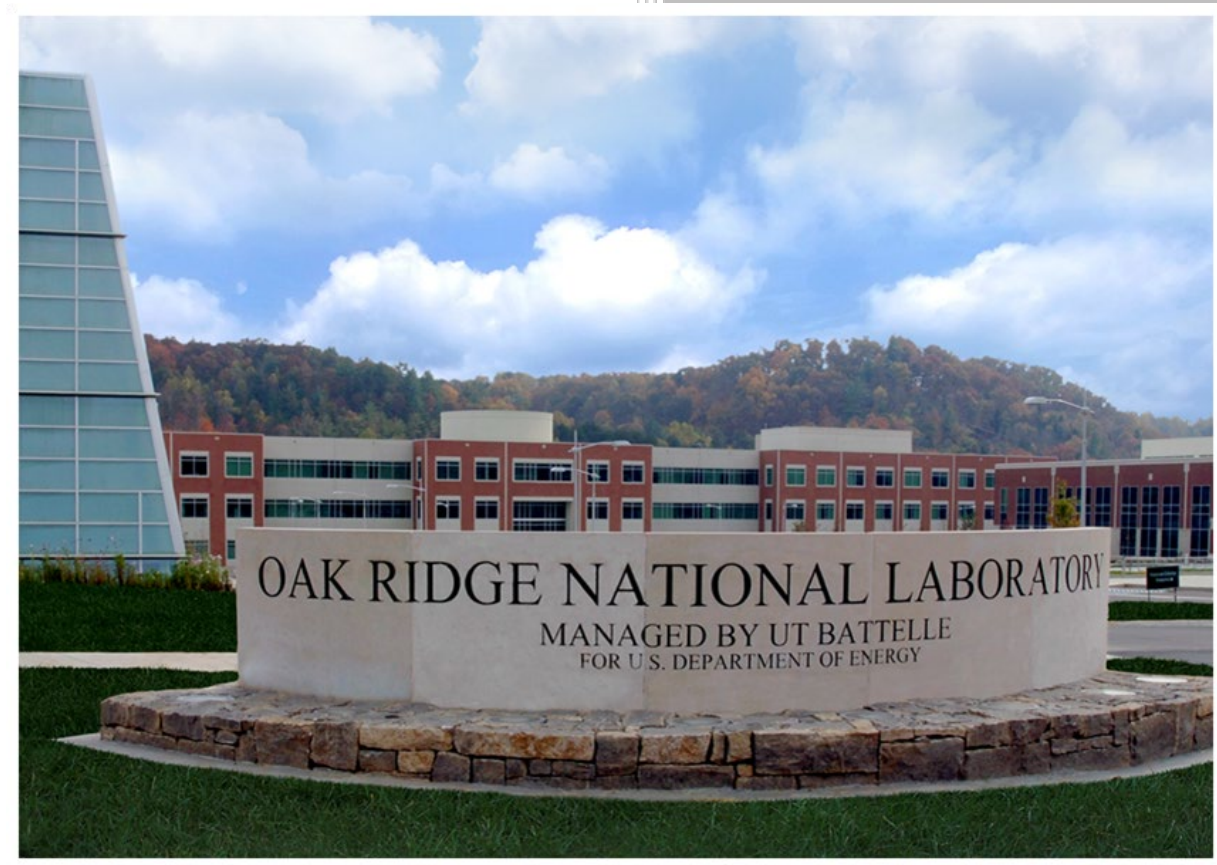

Jim Garner Natalie A. McGirl Logan M. Scott Michael Whitaker

April 2021 


\section{DOCUMENT AVAILABILITY}

Reports produced after January 1, 1996, are generally available free via US Department of Energy (DOE) SciTech Connect.

Website www.osti.gov

Reports produced before January 1, 1996, may be purchased by members of the public from the following source:

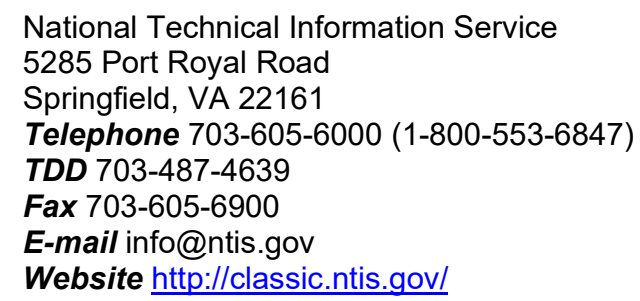

Reports are available to DOE employees, DOE contractors, Energy Technology Data Exchange representatives, and International Nuclear Information System representatives from the following source:

Office of Scientific and Technical Information

PO Box 62

Oak Ridge, TN 37831

Telephone 865-576-8401

Fax 865-576-5728

E-mail reports@osti.gov

Website http://www.osti.gov/contact.html

This report was prepared as an account of work sponsored by an agency of the United States Government. Neither the United States Government nor any agency thereof, nor any of their employees, makes any warranty, express or implied, or assumes any legal liability or responsibility for the accuracy, completeness, or usefulness of any information, apparatus, product, or process disclosed, or represents that its use would not infringe privately owned rights. Reference herein to any specific commercial product, process, or service by trade name, trademark, manufacturer, or otherwise, does not necessarily constitute or imply its endorsement, recommendation, or favoring by the United States Government or any agency thereof. The views and opinions of authors expressed herein do not necessarily state or reflect those of the United States Government or any agency thereof. 
Nuclear Nonproliferation Division

\title{
RESULTS FROM ACCELERATED ENVIRONMENTAL TESTING OF BARCODED
} IDENTIFIERS FOR UF 6 CYLINDERs

\author{
Jim Garner \\ Natalie A. McGirl \\ Logan M. Scott \\ Michael Whitaker
}

April 2021

Prepared by

OAK RIDGE NATIONAL LABORATORY

Oak Ridge, TN 37831-6283

managed by

UT-BATTELLE, LLC

for the

US DEPARTMENT OF ENERGY

under contract DE-AC05-00OR22725 



\section{CONTENTS}

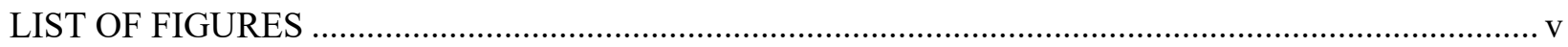

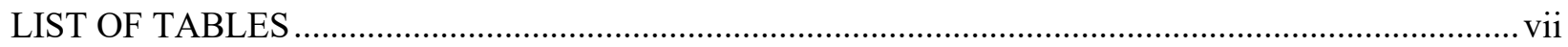

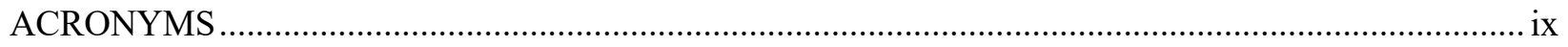

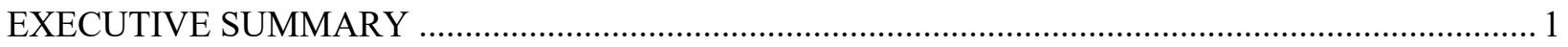

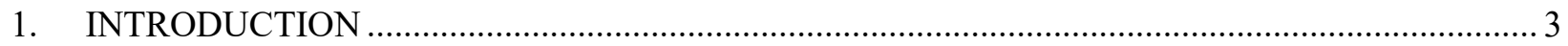

2. PURCHASE AND PREPARATION OF BARCODE SAMPLES …................................................ 4

3. QUANTITATIVE METRIC BARCODE VERIFICATION MEASUREMENT …......................... 7

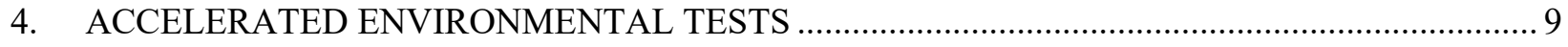

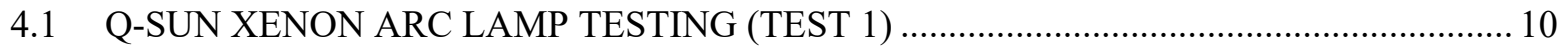

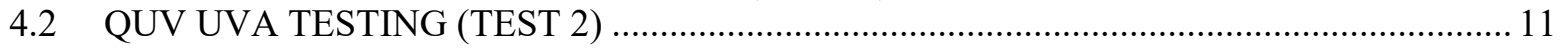

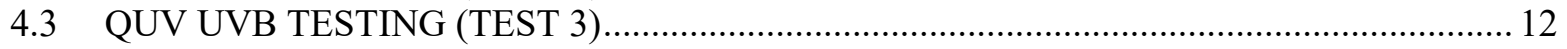

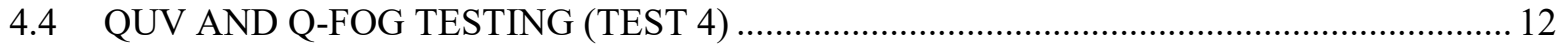

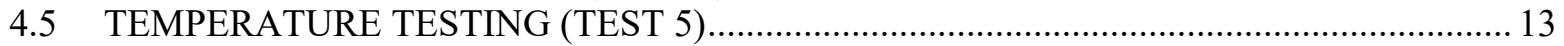

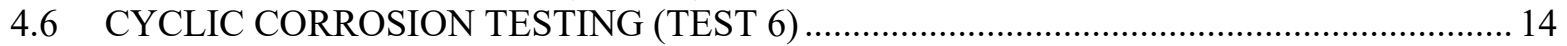

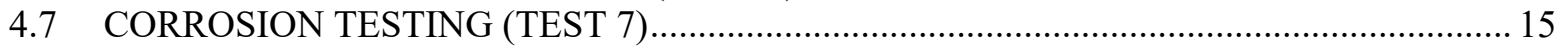

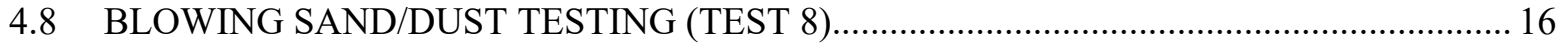

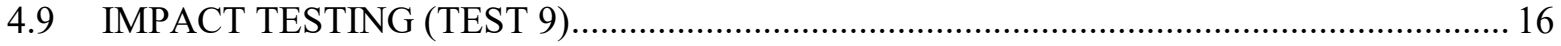

4.10 HIGH PRESSURE AND TEMPERATURE WATER JET TESTING (TEST 10)................. 17

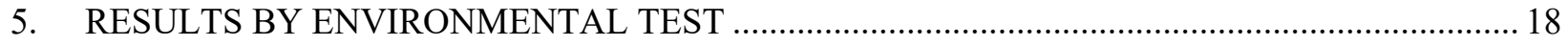

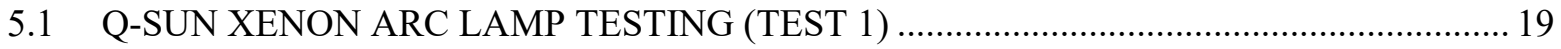

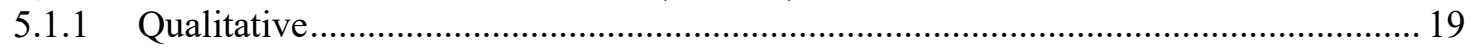

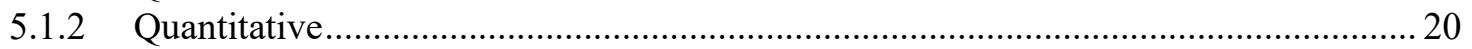

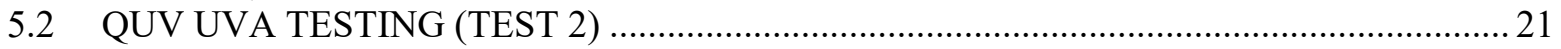

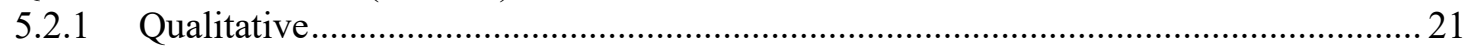

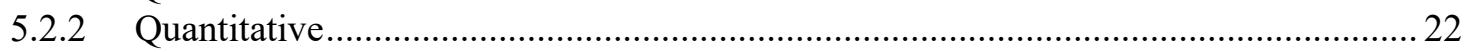

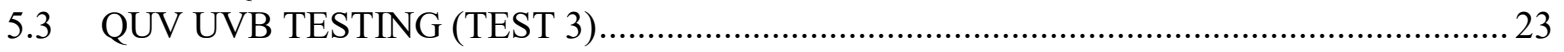

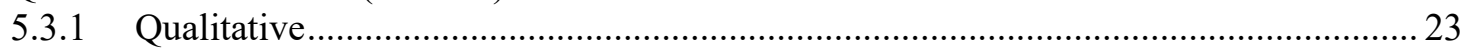

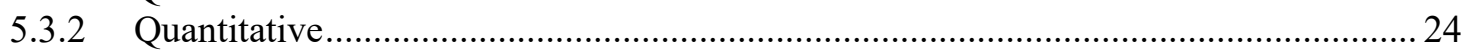

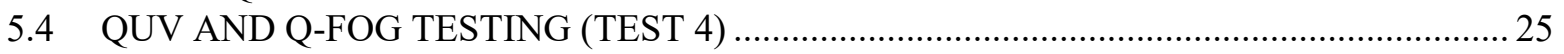

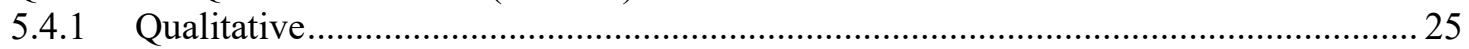

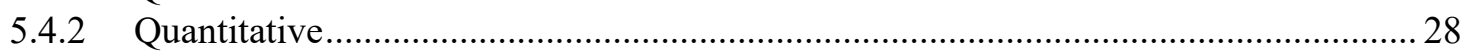

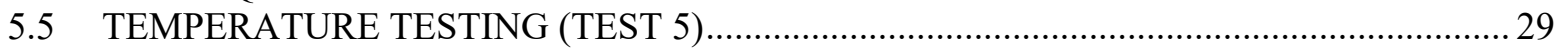

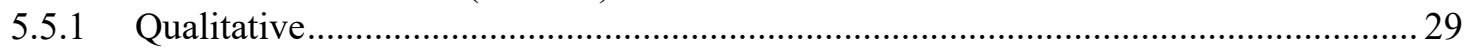

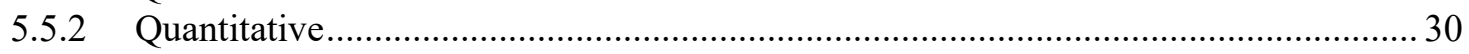

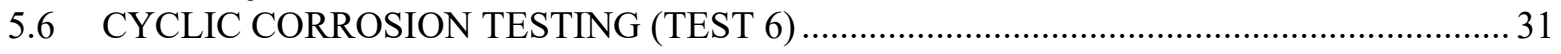

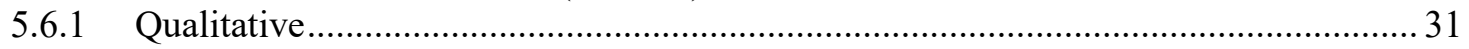

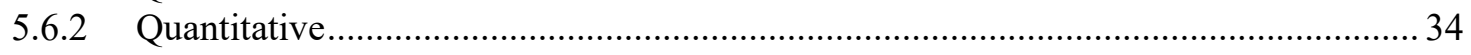

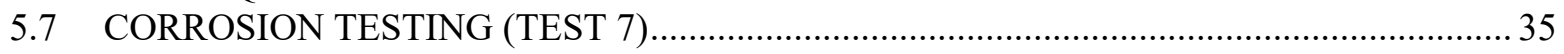

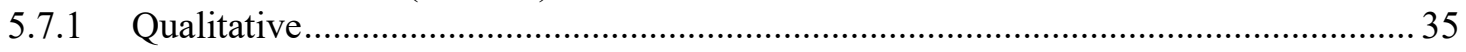

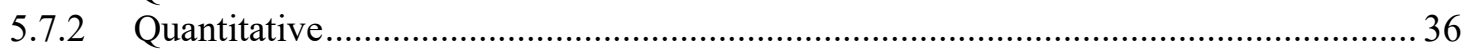

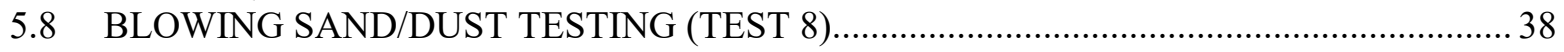

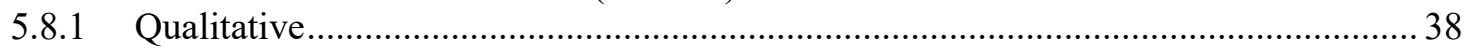

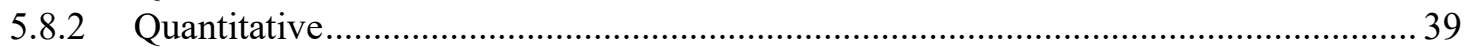

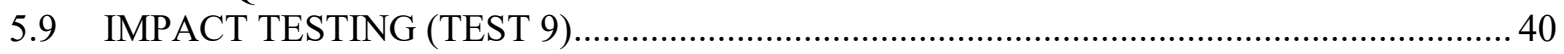

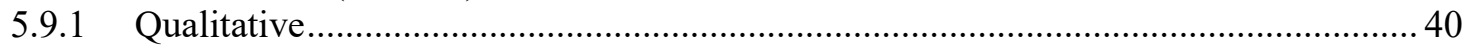

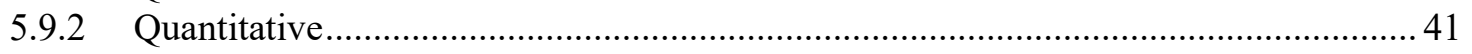

5.10 HIGH PRESSURE AND TEMPERATURE WATER JET (TEST 10) ................................. 42

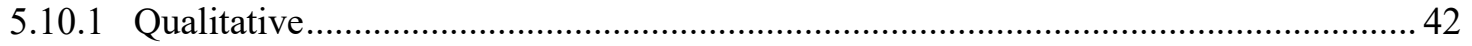




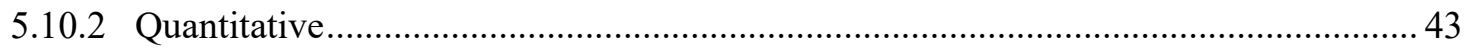

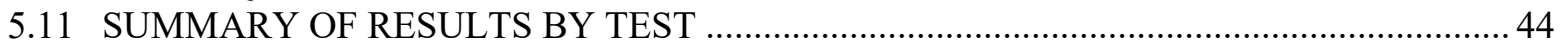

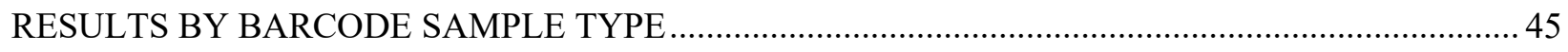

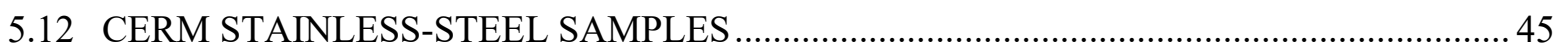

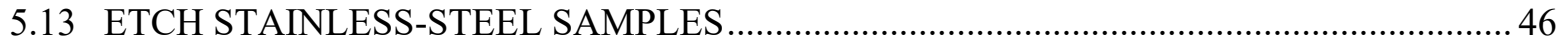

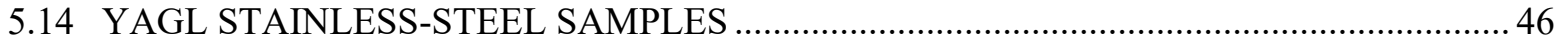

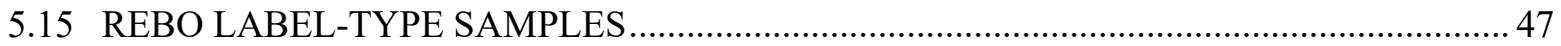

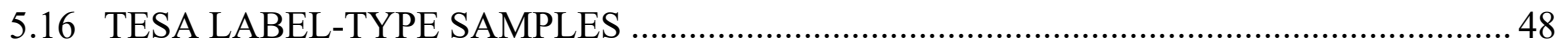

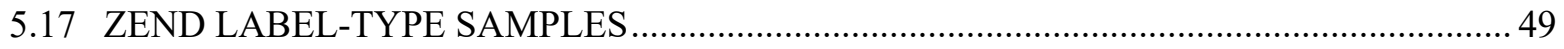

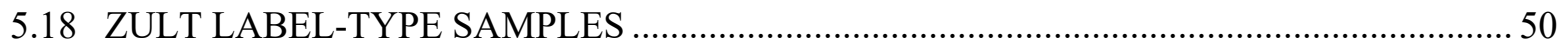

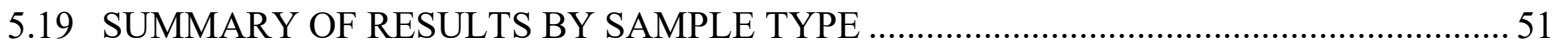

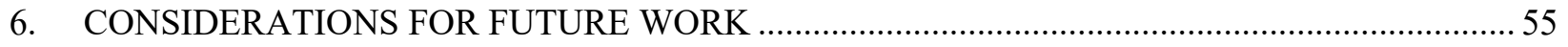

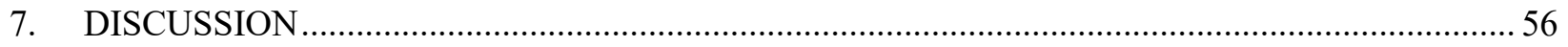

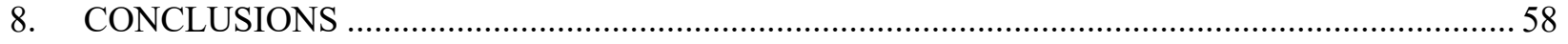

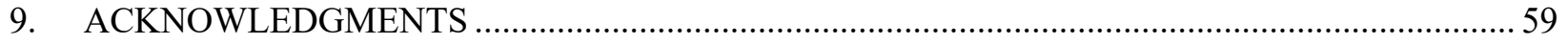

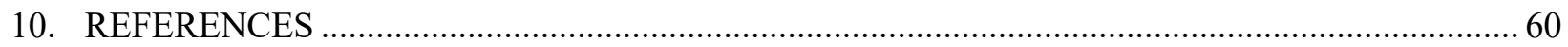

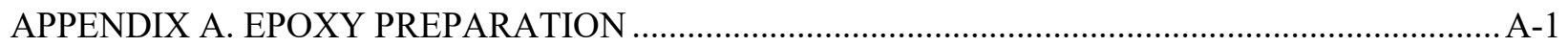

APPENDIX B. BARCODE SAMPLES GROUPED BY SAMPLE TYPE …................................... B-1

APPENDIX C. BARCODE SAMPLES GROUPED BY ENVIRONMENTAL TESTS ...................... C-1 


\section{LIST OF FIGURES}

Figure 1. Examples of QR and Data Matrix 2D barcodes. ................................................................ 3

Figure 2. Multiple standards apply to 2D barcodes and barcode verification......................................... 4

Figure 3. Z-Endure 4000T labels with white specs in the dark modules............................................ 5

Figure 4. Chemically etched stainless-steel barcode sample, no coupon attachment (free)...................... 6

Figure 5. Rebo premium vinyl label self-adhered to a sandblasted coupon. ............................................ 6

Figure 6. Webscan TruCheck DPM Tower. ......................................................................................... 7

Figure 7. Top portion of Webscan Verification Report for YAGL123412 after QUV and Q-FOG

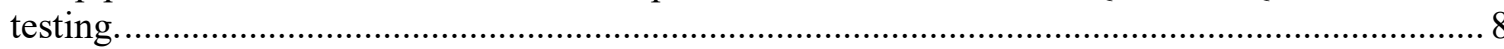

Figure 8. Bottom portion of Webscan Verification Report for YAGL123412 after QUV and Q-

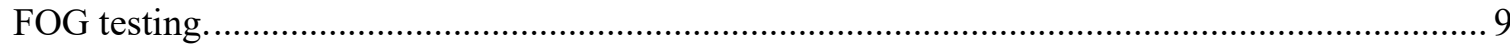

This portion of the report displays the ISO 29158 quality parameters. ................................................. 9

Figure 9. Barcode samples were tested for $500 \mathrm{~h}$ in a Q-SUN Xe-3 Xenon arc lamp chamber................. 10

Figure 10. Barcode samples on Q-SUN Xe-3 Xenon arc lamp chamber loading tray.............................. 11

Figure 11. Barcode samples were initially UVA tested for $1000 \mathrm{~h}$ in a QUV accelerated

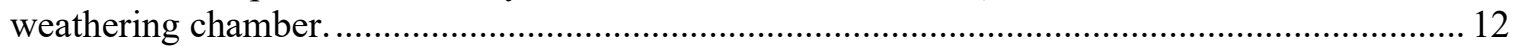

Figure 12. Barcode samples installed in QUV accelerated weathering chamber at test conclusion........... 12

Samples are shown facing out but were turned inward toward UV lamps during testing........................ 12

Figure 13. Barcode samples were tested for $168 \mathrm{~h}$ in a Q-FOG cyclic corrosion (salt fog)

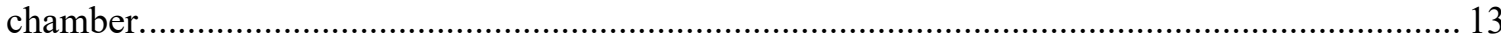

Figure 14. Barcode samples were for 6 weeks in a Tenney TC20RC environmental enclosure................ 14

Figure 15. Barcode samples installed in GTL's salt fog chamber before ambient stage with stress

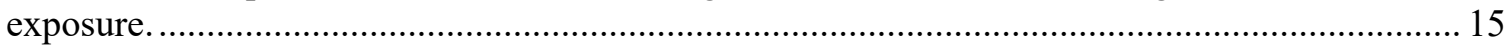

Figure 16. Barcode samples installed in GTL's environmental test chamber before dry stage

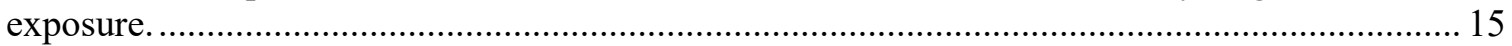

Figure 17. Barcode samples installed in GTL's sand/dust chamber before testing. ............................... 16

Figure 18. Introduction of test sand/dust into GTL's sand/dust chamber............................................. 16

Figure 19. A $500 \mathrm{~g}$ smooth steel sphere is placed inside the guide tube $1,000 \mathrm{~mm}$ above the

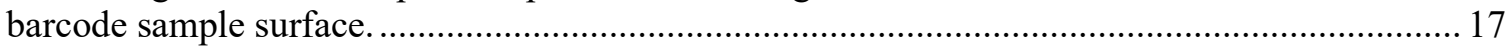

When the pin is removed, the sphere falls freely onto the sample for impact testing.............................. 17

Figure 20. A $500 \mathrm{~g}$ smooth steel sphere on the YAGL123469 barcode sample after impact testing......... 17

Figure 21. Barcode samples subjected to high pressure and temperature water jet testing. ...................... 18

Figure 22. Barcode samples before (left) and after (right) Q-SUN xenon arc lamp testing..................... 19

Figure 23. Cell or symbol contrast for barcode samples before and after Q-SUN xenon arc lamp

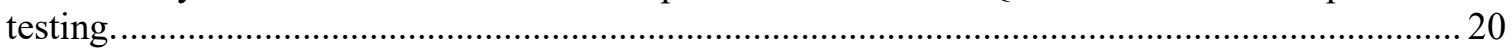

Figure 24. Barcode samples before (left) and after (right) QUV UVA testing.......................................2 21

Figure 25. Cell or symbol contrast for barcode samples before and after QUV UVA testing...................22

Figure 26. Barcode samples before (left) and after (right) UVB testing............................................. 23

Figure 27. Cell or symbol contrast for barcode samples before and after QUV UVB testing.................. 24

Figure 28. Barcode samples before (left) and after (right) QUV and Q-FOG testing. .............................25

Figure 29. Barcode samples installed in Q-FOG cyclic corrosion (salt fog) chamber. ............................. 26

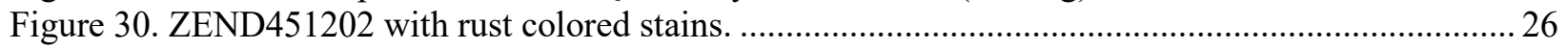

Figure 31. Barcode samples subjected to QUV and Q-FOG testing after hand cleaning........................ 27

Figure 32. ZEND451202 before (left) and after (right) cleaning....................................................... 27

Figure 33. Before exposure, after exposure, and after cleaning cell or symbol contrast for barcode

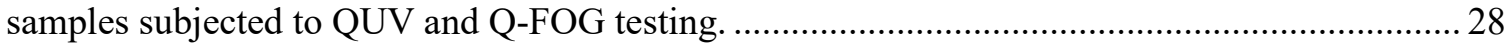

Figure 34. Barcode samples before (top) and after (bottom) temperature testing. .................................... 29

Figure 35. REBO123467 before (left) and after (right) temperature testing. …...................................... 30

Figure 36. Cell or symbol contrast for barcode samples before and after temperature testing................... 30 
Figure 37. Barcode samples before (top) and after (bottom) cyclic corrosion testing.

Figure 38. Barcode samples installed in GTL's salt fog chamber before ambient stage with stress exposure.

Figure 39. Barcode samples subjected to cyclic corrosion testing after hand cleaning.

Figure 40. Before exposure, after exposure, and after cleaning cell or symbol contrast for barcode samples subjected to cyclic corrosion testing.

Figure 41. Barcode samples before (top) and after (bottom) corrosion testing. ...................................... 35

Figure 42. Barcode samples subjected to corrosion testing after cleaning. ........................................... 36

Figure 43. Before exposure, after exposure, and after cleaning cell or symbol contrast for barcode

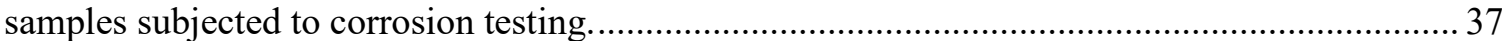

Figure 44. Barcode samples before (top) and after (bottom) blowing sand/dust testing. ......................... 38

Figure 45. Cell or symbol contrast for barcode samples before and after blowing sand/dust

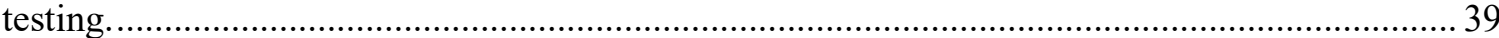

Figure 46. Barcode samples before (top) and after (bottom) impact testing......................................... 40

Figure 47. Cell or symbol contrast for barcode samples before and after impact testing......................... 41

Figure 48. Barcode samples before (top) and after (bottom) high pressure and temperature water

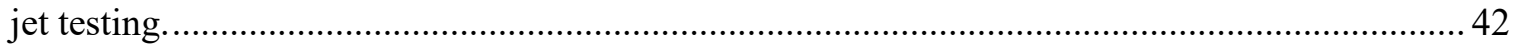

Figure 49. ZULT351099 shown with ripped barcode circled in red.................................................... 43

Figure 50. Cell or symbol contrast for barcode samples before and after high pressure and

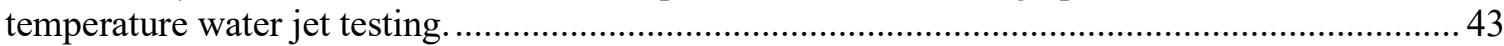

Figure 51. Cell contrast for CerMark-coated laser-marked stainless-steel (CERM) barcode samples before and after all environmental tests.

Figure 52. Cell contrast for chemically etched stainless-steel (ETCH) barcode samples before and after all environmental tests.

Figure 53. Cell contrast for laser-marked stainless-steel (YAGL) barcode samples before and after all environmental tests.

Figure 54. Symbol contrast for Rebo premium vinyl label (REBO) barcode samples before and after all environmental tests.

Figure 55. Cell contrast for laser-marked Tesa tape (TESA) barcode samples before and after all environmental tests.

Figure 56. Symbol contrast for Zebra Z-Endure 4000T acrylic label (ZEND) barcode samples before and after all environmental tests...

Figure 57. Symbol contrast for Zebra Z-Ultimate 3000T polyester label (ZULT) barcode samples

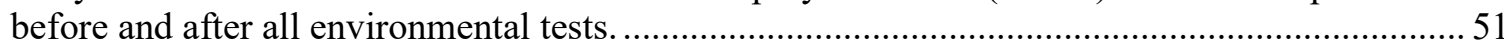

Figure 58. Webscan TruCheck DPM Tower with optical breadboard and sample guides attached........... 56 


\section{LIST OF TABLES}

Table 1. Summary of final contrast values for each sample type. Parentheses indicate negative change in contrast.

Table 4. Summary of cell or symbol contrast values for each test before exposure, after exposure, and after cleaning (sorted by change in contrast).

Table 5. Summary of contrast values for each sample type before exposure, after exposure, and after cleaning. Sorted from largest contrast decline to smallest.

Table 6. Contrast values for CERM samples before exposure, after exposure, and after cleaning for each test.

Table 7. Contrast values for ZEND samples before exposure, after exposure and after cleaning for each test.

Table 8. Contrast values for ZULT samples before exposure, after exposure and after cleaning for each test.

Table 9. Contrast values for TESA samples before exposure, after exposure, and after cleaning for each test.

Table 10. Contrast values for REBO samples before exposure, after exposure, and after cleaning for each test.

Table 11. Contrast values for ETCH samples before exposure, after exposure, and after cleaning for each test.

Table 12. Contrast values for YAGL samples before exposure, after exposure, and after cleaning for each test.....

Table 13. Strengths and weaknesses of sample types exposed to environmental testing.

Table 14. Summary of samples tested and conclusions based on quantitative and qualitative analysis of the results. 



\section{ACRONYMS}

2D two-dimensional

CERM CerMark-coated laser-marked stainless-steel sample

DPM direct-part-marking

ETCH chemically etched stainless-steel sample

GTL Global Testing Laboratories

INMM Institute of Nuclear Materials Management

ORNL Oak Ridge National Laboratory

QR

REBO

TESA

quick response

Rebo premium vinyl label

laser-marked Tesa tape sample

WNTI World Nuclear Transport Institute

YAGL laser-marked stainless-steel sample

ZEND Zebra Z-Endure 4000T acrylic label

ZULT Zebra Z-Ultimate 3000T polyester label 



\section{EXECUTIVE SUMMARY}

Researchers at Oak Ridge National Laboratory (ORNL) have investigated the applicability of direct part marking techniques and barcode specifications for metal nameplates attached to $\mathrm{UF}_{6}$ cylinders. Testing in 2016 and 2017 evaluated how the size of the barcode, read distance, read angle, surface finish of the material, and marking technique influenced barcode readability as measured by commercial off-the-shelf barcode readers. This work concluded that ball-blasted stainless-steel sheets laser marked with CerMark laser marking ink offered a combination that was readable for the nominal use case. This combination of metal, surface finish of the material, and marking technique was recommended in the "Standard for UF 6 Cylinder Identification" issued in 2017 by the World Nuclear Transport Institute (WNTI).

As industry has begun to add two-dimensional barcodes to cylinders, questions are being raised regarding the readability of the barcodes over the life span of a cylinder which can exceed 40 years. To address these questions, ORNL researchers designed and conducted an accelerated environmental testing campaign to produce the environmental conditions that $\mathrm{UF}_{6}$ cylinders often experience during typical operational and storage conditions (e.g., extreme heat, high humidity, etc.).

In planning the accelerated testing campaign, the researchers decided to evaluate six marking methods in addition to the marking method recommended in the WNTI standard for the stainless-steel nameplate, including two different methods for marking stainless steel and four types of adhesive labels. The seven sample types that were evaluated included:

- CerMark-coated laser-marked stainless-steel samples (CERM)

- Chemically etched stainless-steel samples (ETCH)

- Laser-marked stainless-steel samples (YAGL)

- Rebo premium vinyl labels (REBO)

- Laser-marked Tesa tape samples (TESA)

- Zebra Z-Endure 4000T acrylic labels (ZEND)

- Zebra Z-Ultimate 3000T polyester labels (ZULT)

This report provides the qualitative and quantitative results from the accelerated environmental testing campaign. A total of 444 samples were subjected to 10 tests that included UV, salt fog, temperature, blowing dust, high pressure and temperature water, and impact exposure. Each sample included a twodimensional barcode and an alphanumeric string representing the cylinder identification number. When evaluating the results, primary attention was placed on the decodability of the barcodes using a barcode verifier device, since machine scanning a barcode offers the greatest efficiency and accuracy gains when reading cylinder identification numbers during safeguards activities. Contrast was considered the critical test parameter because maintaining contrast under a wide range of environmental conditions is of fundamental importance to decoding the identification number of the barcodes. Additionally, manual readability of the alphanumeric string was considered an important factor in determining whether an inspector or operator could still read the identification number of the cylinder if the barcode proved to be undecodable.

Table 1 provides a summary of the change in contrast values for each sample type after environmental testing. The contrast on average for the CerMark laser marking ink (CERM) samples, as recommended in the WNTI standard, actually improved after exposure. Zebra Z-Endure 4000T acrylic labels (ZEND) also performed very well and can be affixed to a substrate by a peel and stick application, which makes them an attractive alternative to welding or epoxying stainless-steel sheets onto a substrate. 
Table 1. Summary of final contrast values for each sample type. Parentheses indicate negative change in contrast.

\begin{tabular}{cc}
\hline Sample Type & Final Difference \\
\hline CERM & 1.66 \\
ZEND & 0.98 \\
ZULT & $(1.51)$ \\
TESA & $(1.81)$ \\
REBO & $(2.30)$ \\
ETCH & $(2.77)$ \\
YAGL & $(3.25)$ \\
\hline
\end{tabular}

It is important to note that all the samples could be manually read after each test. In the situations where the barcode verifier could not decode the barcode, the researcher could still manually read the alphanumeric string representing the cylinder identification number. Manual readability was thus maintained as a back-up method to read the identification numbers, albeit taking more time and introducing accuracy issues such as transposition of numbers or letters when manually recording the identification number.

Importantly, the testing did show significant differences with regard to machine decodability under the test conditions, specifically with respect to maintenance of contrast. Table 13 and Table 14 in this report summarize the results for machine decodability; Table 13 summarizes the major advantages and weaknesses of each sample type across all the tests and Table 14 presents recommendations for preferred samples based on the testing results. As presented in these tables, the ball-blasted stainless-steel sheets laser marked with CerMark laser marking ink (CERM) is the most highly recommended material and marking technique identified in this testing campaign for supplemental $\mathrm{UF}_{6}$ cylinder identifiers, as measured by the highest average change in cell contrast after environmental testing. Note that corrosion tests represented a weakness for CERM samples, though in all cases, the barcode was decoded and the alphanumeric string could be read by the researcher.

Additionally, both the Zebra Z-Endure 4000T acrylic labels (ZEND) and Zebra Z-Ultimate 3000T polyester labels (ZULT) are recommended alternatives to stainless-steel supplemental $\mathrm{UF}_{6}$ cylinder identifiers due to their consistent symbol contrast values before and after environmental testing. While ZEND labels overall had higher symbol contrast values and a longer outdoor use rating by the manufacturer compared to ZULT labels (10 years compared to 3 years), ZEND labels must be special ordered and they exhibited printing flaws during the label creation process.

The results from the accelerated testing campaign point to a set of options for applying the WNTI standard to either newly fabricated cylinders or previously fabricated cylinders currently being used. The results reaffirm the recommendation in the WNTI standard to use a Data Matrix barcode laser-marked with CerMark laser marking ink onto a ball-blasted stainless-steel plate for new cylinders in which the identification could be marked onto the stainless-steel nameplate during fabrication. For existing cylinders, several label-type samples successfully endured the testing and offer an inexpensive, rapidlydeployable approach to applying the WNTI-recommended identification format in a manner that does not required welding or epoxying on a supplemental metal plate. 


\section{INTRODUCTION}

Since 2011, researchers at Oak Ridge National Laboratory (ORNL) have been investigating how including machine-readable $\mathrm{UF}_{6}$ cylinder identification features could enhance safeguards at facilities handling $\mathrm{UF}_{6}$ cylinders. As reported in the 2017 Institute of Nuclear Materials Management paper by Garner et al. [1], the barcode size and marking technique can impact the range over which commercial off-the-shelf barcode readers can successfully decode barcodes. The 2017 paper concluded that a 1.4 in. Data Matrix barcode laser-marked with CerMark laser marking ink onto a ball-blasted stainless-steel plate would be very suitable for representative use cases involving a $\mathrm{UF}_{6}$ cylinder global identifier. These recommendations were subsequently incorporated into the 2017 World Nuclear Transport Institute "Standard for $\mathrm{UF}_{6}$ Cylinder Identification" [2].

This earlier work focused on Data Matrix two-dimensional (2D) barcodes (Figure 1). Data Matrix and Quick Response (QR) are two of the most widely used 2D barcode symbologies and can be printed on labels or directly marked on parts. The specifications and quantifiable grading procedures for these 2D barcodes are covered by several standards:

- ISO/IEC 16022, "Data Matrix bar code symbology specification” [3]

- ISO/IEC 18004, “QR code bar code symbology specification” [4]

- ISO/IEC 15415, "2-D bar code print quality standard [5]," which incorporated and expanded upon marking quality definitions from ISO/IEC 16022 and ISO/IEC 18004

- AIM DPM-1-2006, verification standard for direct part marking (DPM) 2D code image quality established by the Automatic Identification Manufacturers based on ISO/IEC 15415:2004

- ISO/IEC TR 29158, verification standard for DPM 2D code image quality adopted by International Organization for Standardization [6], which was based on AIM DPM-1-2006 and incorporated ISO/IEC15415:2011

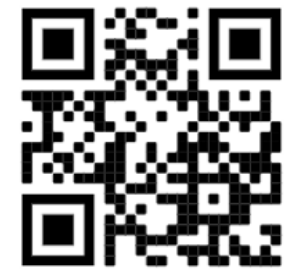

QR Code

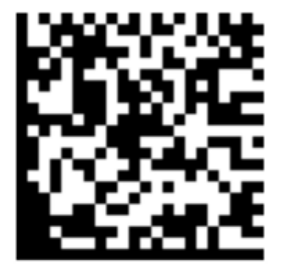

Data Matrix

Figure 1. Examples of $Q R$ and Data Matrix 2D barcodes.

Figure 2 illustrates the relationship between the standards that govern 2D barcodes. Data Matrix barcodes are considered better than QR codes for industrial applications because they have higher error correction. Many 2D barcode symbologies include error correction. The $14 \times 14$ module Data Matrix barcodes, as recommended by World Nuclear Transport Institute, include $28 \%-39 \%$ error correction [7]. QR codes have four error correction levels, but they have a maximum of $30 \%$ error correction. 


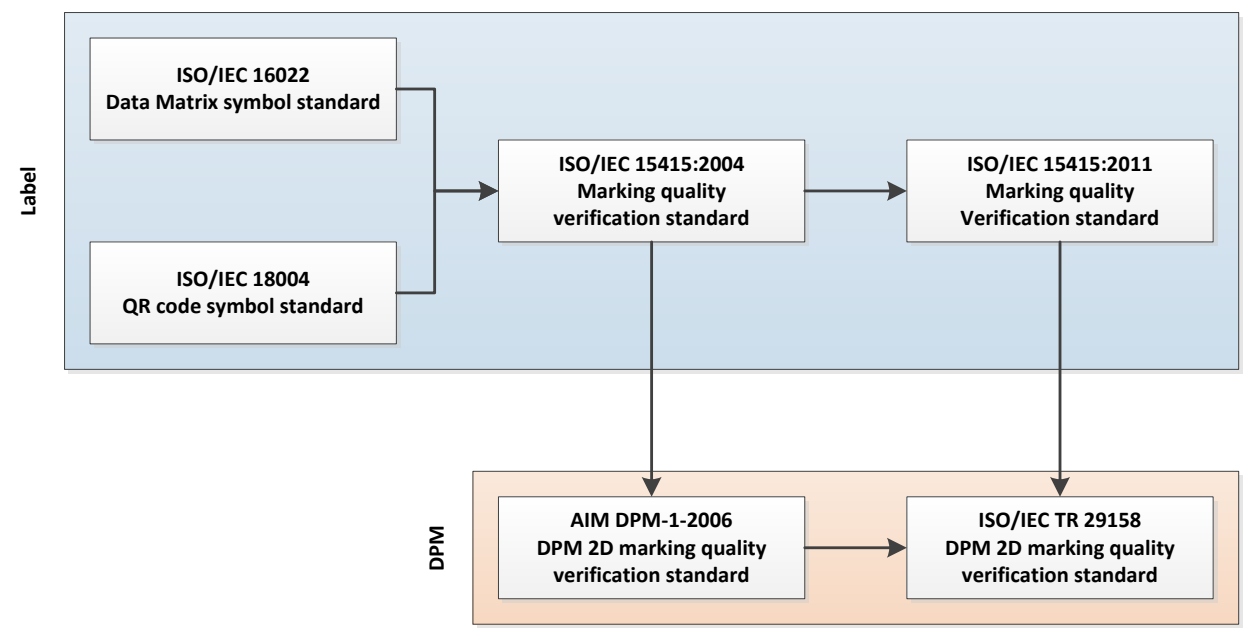

Figure 2. Multiple standards apply to 2D barcodes and barcode verification.

\section{PURCHASE AND PREPARATION OF BARCODE SAMPLES}

The ORNL team collected and prepared approximately 630 barcode samples and labels for accelerated environmental testing. Seven different types of barcoded identifiers were evaluated:

- CerMark-coated laser-marked stainless-steel samples (CERM)

- Chemically etched stainless-steel samples (ETCH)

- Laser-marked stainless-steel samples (YAGL)

- Rebo premium vinyl labels (REBO)

- Laser-marked Tesa tape samples (TESA)

- Zebra Z-Endure 4000T acrylic labels (ZEND)

- Zebra Z-Ultimate 3000T polyester labels (ZULT)

The CERM samples were purchased from Automation-Plus at a cost of $\$ 22.00$ each for a quantity of 100 . These samples were prepared from 13 gauge (0.0897 in.) 304 stainless sheet that was ball blasted, coated with the CerMark laser marking ink, laser marked using a YAG laser system, and then cleaned to remove the unbonded material.

The ETCH samples were purchased from American Nameplate at a cost of $\$ 23.50$ each for a quantity of 100 plus. These samples were prepared from 13 gauge $(0.0897$ in.) 304 stainless sheet that was ball blasted by Automation-Plus then shipped to American Nameplate as $18 \times 16$ in. sheets. The sheets were then chemically etched to a depth of approximately 0.005 in. Paint was applied to the surface and squeegeed to leave paint only in the etched markings. The samples were then cut from the larger sheets using a water jet.

The YAGL samples were also purchased from Automation-Plus at a cost of $\$ 17.50$ each for a quantity of 100. These samples were prepared from 13 gauge (0.0897 in.) 304 stainless sheet that was ball blasted and then laser marked using a YAG laser system.

The REBO labels were provided complementary by ZingGreen Safety Products, one of the U.S. distributors for the REBO printers. The vendor used a Rebo thermal printer (e.g., SMS430) with Rebo Premium Industrial Vinyl labels (i.e., ST700) and industrial print ribbon (i.e., SR10). 
The TESA samples were purchased from Code Source at a cost of $\$ 4.36$ each for a quantity of 100 . These labels were manufactured from Tesa Secure 6973 tape, which is a $118 \mu \mathrm{m} / 4.6 \mathrm{mil}$ thick double layer, brittle, acrylic film with $25 \mathrm{~g} / \mathrm{m}^{2}$ resin modified acrylic adhesive [8]. The vendor promoted these labels as being "as robust as metal" once applied.

ORNL researchers printed the ZEND samples using a Zebra ZT410 thermal transfer printer onto Zebra ZEndure 4000T acrylic label material using Zebra 5100 Premium ribbon. The manufacturer markets ZEndure $4000 \mathrm{~T}$ as a special label material offering 10-year outdoor durability. This material is typically only available by special order and is subject to nonrecurring engineering charges (e.g., die fee) and a minimum purchase quantity. If these obstacles can be surmounted, we estimate the cost for a $2 \times 4$ in. label to be approximately $\$ 0.15$ each. The Z-Endure 4000T material is a 2.0 mil acrylic with a 0.8 mil acrylic adhesive. The Z-Endure $4000 \mathrm{~T}$ is advertised to offer a service temperature of $-40^{\circ} \mathrm{F}$ to $212^{\circ} \mathrm{F}$ $\left(-40^{\circ} \mathrm{C}\right.$ to $\left.100^{\circ} \mathrm{C}\right)$. The manufacturer reports testing this material using a QUV accelerated weathering tester for 10,000 hours alternating between 8 hours of UV exposure at $60^{\circ} \mathrm{C}$ and 4 hours of condensation at $50^{\circ} \mathrm{C}$. The manufacturer reported no degradation after 5,000 hours and very slight chalking after 10,000 hours. This test procedure appears similar to Tests 2 and 3 described in this report, but the manufacturer does not include sufficient details about the type of UV bulb or irradiance to directly compare the manufacturer's results with this report's results. [9]

Note that the authors had trouble printing faultless barcodes using the Zebra Z-Endure 4000T acrylic label material. As shown in Figure 3 white streaks were observed in the dark modules, especially in the finder pattern shown on the left of the Data Matrix barcode.

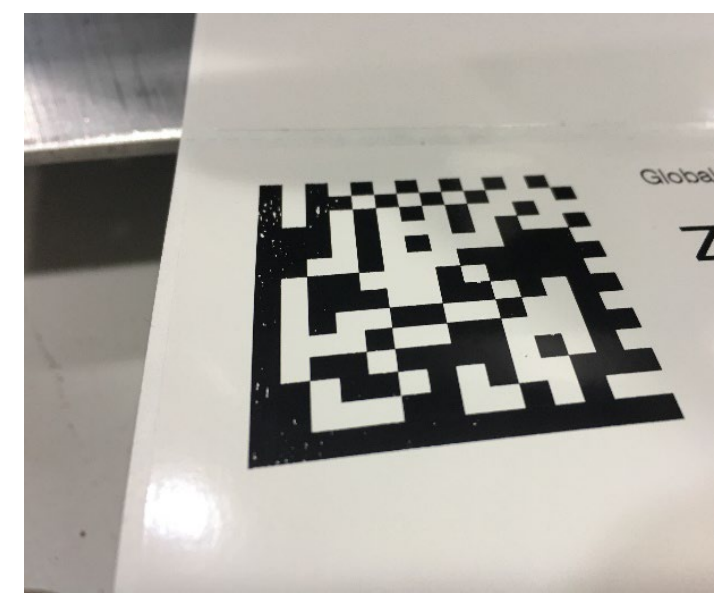

Figure 3. Z-Endure 4000T labels with white specs in the dark modules. These labels were printed at ORNL using a Zebra ZT410 thermal transfer printer with 5100 premium resin.

The researchers printed the ZULT samples using a Zebra ZT410 thermal transfer printer onto Zebra ZUltimate 3000T acrylic label material using Zebra 5100 Premium ribbon. These samples could be purchased preprinted for approximately $\$ 0.27$ each for a quantity of 100 or printed on-premise for about $\$ 0.08$ each excluding the cost of the $\sim \$ 1,500$ printer. Z-Ultimate $3000 \mathrm{~T}$ is 2.0 mil polyester with 0.8 mil acrylic-based adhesive. The manufacturer advertises the Z-Ultimate has a 3 year expected exterior life with a service temperature from $-40^{\circ} \mathrm{F}$ to $302^{\circ} \mathrm{F}\left(-40^{\circ} \mathrm{C}\right.$ to $\left.150^{\circ} \mathrm{C}\right)$ [10]. Despite using the same printer and the same printing ribbon, the authors did not observe the white flecks in ZULT samples that the authors experienced with the ZEND samples.

Of the 630 barcode samples that were prepared, 444 were selected for environmental testing; the remaining 186 barcode samples were saved as backups. Most of these barcode samples were welded, epoxied, or self-adhered to $1 / 2$ in. thick A516 steel coupons, which is the same steel alloy and thickness 
used to manufacture model 30B UF 6 cylinders. ORNL purchased $4 \mathrm{ft} \times 8 \mathrm{ft}$ A516 sheets with a mill finish, and ORNL machinists laser-cut the sheets into smaller 3 in. $\times 6$ in. coupons. All coupons were then sandblasted, and a subset of the sandblasted coupons were further polished to fully remove the mill finish for welding.

Of the stainless-steel barcode samples (CERM, ETCH, YAGL), 45 were welded to polished coupons, and 116 were affixed to the coupons using Aremco 517 epoxy. ${ }^{1}$ The epoxy preparation process is described in Appendix A. Of the 116 stainless-steel barcode samples epoxied to the coupons, 19 were epoxied to polished coupons, and 97 were epoxied to sandblasted-only coupons. The remaining 105 stainless-steel barcode samples that were not attached to coupons were considered "free."

The label-type barcode samples (REBO, TESA, ZEND, ZULT) were equipped with an adhesive backing. Twenty-four of the label-type barcode samples were self-adhered to polished coupons and 154 were selfadhered to sandblasted-only coupons. There were no welded or "free" label-type barcode samples. A selection of stainless-steel and label-type barcode samples are shown in Figure 4 and Figure 5, respectively.

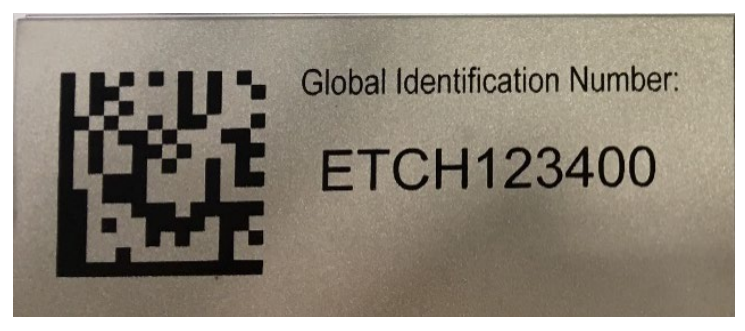

Figure 4. Chemically etched stainless-steel barcode sample, no coupon attachment (free).

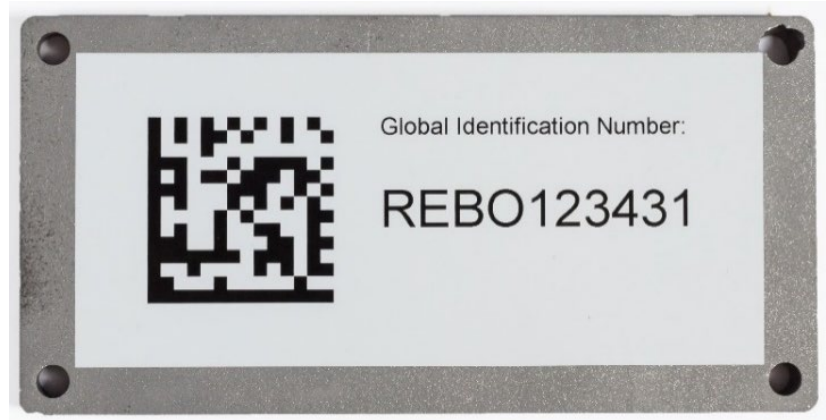

Figure 5. Rebo premium vinyl label self-adhered to a sandblasted coupon.

The number of tested stainless-steel (CERM, ETCH, YAGL) and label-type (REBO, TESA, ZEND, ZULT) barcode samples prepared using each coupon attachment method is provided in Table 2 . The identification number, environmental test information, and coupon attachment method for individual samples are displayed in Appendix B.

\footnotetext{
${ }^{1}$ This epoxy has been used by other $\mathrm{UF}_{6}$ industry members to adhere new placards to the skirt of cylinders. This type of epoxy may be an attractive alternative for industry compared to welding because it may be a permanent way to adhere the global identifier to the front face of $\mathrm{UF}_{6}$ cylinders without requiring an R-stamp welder during the recertification process.
} 
Table 2. Number of Samples tested for each barcode types and coupon attachment method combination.

\begin{tabular}{lccc|cccc}
\hline \multirow{2}{*}{\begin{tabular}{c} 
Coupon Finish \\
\multicolumn{1}{c}{ (Attachment Method) }
\end{tabular}} & \multicolumn{6}{c}{ Barcode Sample Type } \\
\cline { 2 - 8 } & \multicolumn{2}{c}{ Stainless Steel (266 total) } & \multicolumn{4}{c}{ Label (178 total) } \\
\cline { 2 - 8 } & CERM & ETCH & YAGL & REBO & TESA & ZEND & ZULT \\
\hline Polished (Welded) & 15 & 15 & 15 & 0 & 0 & 0 & 0 \\
Polished (Epoxied) & 7 & 6 & 6 & 0 & 0 & 0 & 0 \\
Polished (Self-Adhered) & 0 & 0 & 0 & 6 & 6 & 6 & 6 \\
Sandblasted-Only (Epoxied) & 31 & 33 & 33 & 0 & 0 & 0 & 0 \\
Sandblasted-Only (Self-Adhered) & 0 & 0 & 0 & 42 & 42 & 28 & 42 \\
Free & 34 & 33 & 38 & 0 & 0 & 0 & 0 \\
\hline Total Number of Samples & 87 & 87 & 92 & 48 & 48 & 34 & 48 \\
\hline
\end{tabular}

\section{QUANTITATIVE METRIC BARCODE VERIFICATION MEASUREMENT}

The ORNL team used a Webscan TruCheck DPM Tower to scan the barcode samples (Figure 6). The DPM Tower is a barcode verifier that grades 2D barcodes directly marked on a sample using ISO 29158 (AIM-DPM) and 2D barcodes printed on label material using ISO 15415. The chemically etched (ETCH), laser-marked (YAGL, TESA), and CerMark-coated laser-marked (CERM) barcodes were DPM-type barcodes and were thus graded using ISO 29158, but the Rebo (REBO) and Zebra (ZEND, ZULT) barcode samples were graded using ISO 15415.

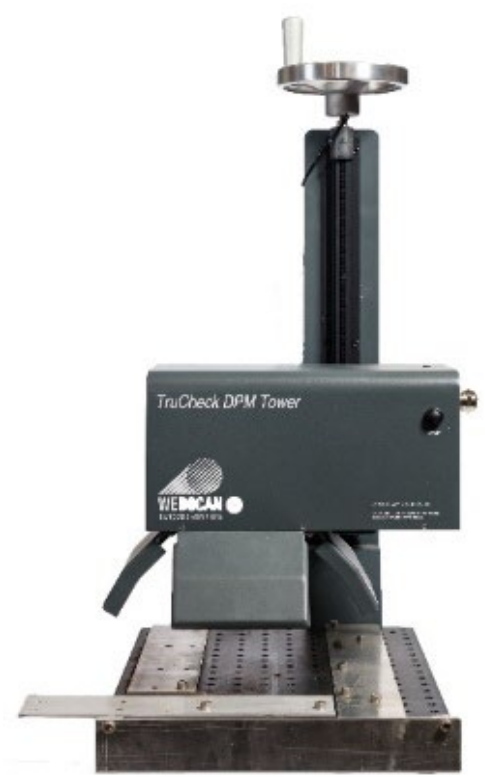

Figure 6. Webscan TruCheck DPM Tower.

ORNL configured the barcode verifier to produce a PDF report and a .CSV summary file for each scan. As shown in Figure 7, the PDF report includes summary information at the top that provides the data, symbology, and grades for any tests selected. 


\begin{tabular}{|c|c|c|c|c|c|c|}
\hline WEBSCAN & \multicolumn{5}{|c|}{$\begin{array}{c}\text { Software Version: 3.03.54, Unit Serial: TC-825-0318-121 } \\
\text { Verified: Tue 16-Apr-2019 03:01:04 PM, Last Calibrated: Tue 16-Apr-2019 11:59:30 AM } \\
\text { Page } 1 \text { of } 2\end{array}$} & \\
\hline \multicolumn{7}{|c|}{ Report Summary } \\
\hline \begin{tabular}{|l|} 
Data \\
Symbology
\end{tabular} & \multicolumn{6}{|l|}{\begin{tabular}{|l|} 
YAGL123412 \\
DataMatrix
\end{tabular}} \\
\hline Verified By & \multicolumn{6}{|l|}{ ORNL_Admin } \\
\hline \multicolumn{7}{|c|}{ Verification Grades } \\
\hline Standard & Grade & Aperture & Wavelength & Lighting & Formal Grade & Notes \\
\hline ISO15415 & $F(0.0)$ & 20 & 660 & 45 & $0.0 / 20 / 660 / 45$ & \\
\hline $\begin{array}{l}\text { ISO29158 } \\
\text { (AIM-DPM) }\end{array}$ & C $(2.0)$ & 81 & 660 & $45 \mathrm{Q}$ & $\begin{array}{c}\text { DPM } \\
2.0 / 81 / 660 / 45 Q \\
\end{array}$ & $\begin{array}{c}\text { [Warning]Symbol } \\
X \text { X-Dimension out of } \\
\text { range }\end{array}$ \\
\hline
\end{tabular}

Figure 7. Top portion of Webscan Verification Report for YAGL123412 after QUV and Q-FOG testing.

ORNL recorded results for both ISO 15415 and ISO 29158 for each sample; however, the ISO 15415 results are only meaningful for the Rebo and Zebra label barcode samples (REBO, ZEND, and ZULT), and the ISO 29158 results are only meaningful for the DPM barcode samples (CERM, ETCH, YAGL, and TESA). Figure 8 shows an example ISO 29158 results for a laser-marked stainless-steel sample (YAGL123412) that was part of Test 4 (QUV and Q-FOG testing). Details about the key metrics that changed for YAGL123412 after environmental exposure are discussed in Garner et al. [11]. 


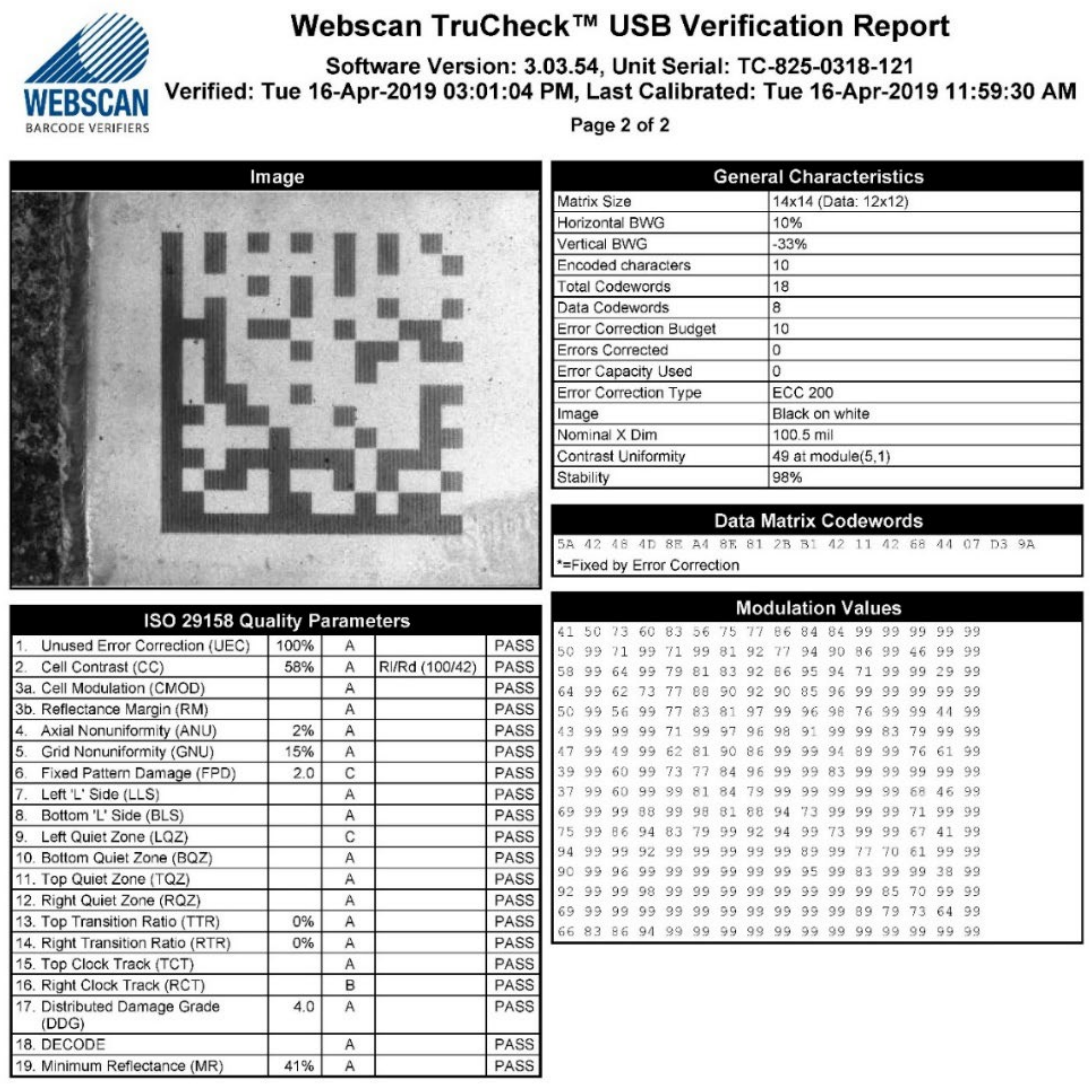

Figure 8. Bottom portion of Webscan Verification Report for YAGL123412 after QUV and Q-FOG testing. This portion of the report displays the ISO 29158 quality parameters.ACCELERATED ENVIRONMENTAL TESTS

A series of 10 tests were designed to produce environmental conditions that $\mathrm{UF}_{6}$ cylinders may experience during their life cycle which can exceed 40 years. As summarized in Table 3 and further described in the following sections, four tests were conducted at Q-Lab in Homestead, Florida, one test was conducted at ORNL, and five tests were conducted at Global Testing Laboratories (GTL) in Knoxville, Tennessee.

Table 3. Environmental Test Summary.

\begin{tabular}{|c|c|c|c|c|c|c|}
\hline \multirow{2}{*}{$\begin{array}{c}\text { Test } \\
\text { Number }\end{array}$} & \multirow{2}{*}{ Environmental Test } & \multirow{2}{*}{$\begin{array}{c}\text { Testing } \\
\text { Location }\end{array}$} & \multirow{2}{*}{$\begin{array}{l}\text { Dates } \\
\text { (2019) }\end{array}$} & \multicolumn{3}{|c|}{ Number of Samples } \\
\hline & & & & Stainless-Steel & Label-Type & Total \\
\hline 1 & Q-SUN Xenon Arc Lamp & Q-Lab & Feb.-Mar. & 10 & 12 & 22 \\
\hline 2 & QUV UVA & Q-Lab & Feb.-Mar. & 12 & 12 & 24 \\
\hline 3 & QUV UVB & Q-Lab & Feb.-Mar. & 12 & 12 & 24 \\
\hline 4 & QUV and Q-FOG & Q-Lab & Feb.-Mar. & 12 & 12 & 24 \\
\hline 5 & Temperature & ORNL & Feb.-Apr. & 37 & 24 & 61 \\
\hline 6 & Cyclic Corrosion & GTL & Jan.-Jun. & 41 & 22 & 63 \\
\hline 7 & Corrosion & GTL & Jan.-Jun. & 36 & 21 & 57 \\
\hline 8 & Blowing Sand/Dust & GTL & Jan.-Jun. & 37 & 20 & 57 \\
\hline 9 & Impact & GTL & Jan.-Jun. & 34 & 21 & 55 \\
\hline
\end{tabular}




\subsection{Q-SUN XENON ARC LAMP TESTING (TEST 1)}

Q-Lab staff installed 22 barcode samples into a Q-SUN Xe-3 xenon arc lamp chamber as shown in Figure 9 and Figure 10. This chamber exposed samples to light with humidity control and a water spray, replicating sunlight and rain conditions. Testing followed a cycle like Cycle 1 in ASTM G155, "Standard Practice for Operating Xenon Arc Light Apparatus for Exposure of Non-Metallic Materials.” An irradiance of $0.55 \mathrm{~W} / \mathrm{m}^{2}$ at $340 \mathrm{~nm}$ and the "daylight - Q" filters were used [12]. Samples were exposed to $102 \mathrm{~min}$ of light at $63^{\circ} \mathrm{C}$ black panel temperature, then to $18 \mathrm{~min}$ of light and water spray. This cycle was repeated for a total $500 \mathrm{~h}$. A comprehensive list of samples subjected to Q-SUN xenon arc lamp testing is included in Appendix C.

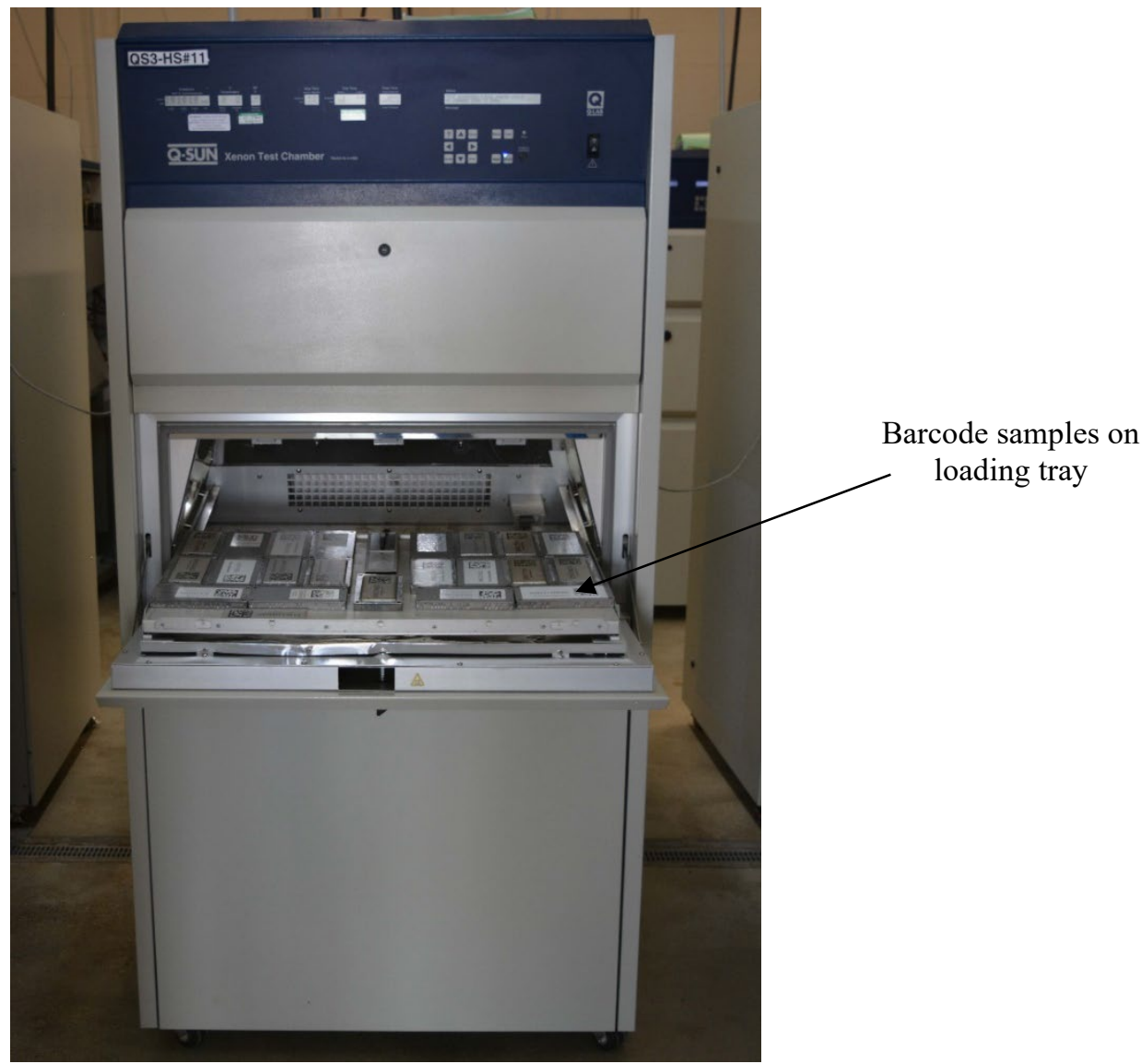

Figure 9. Barcode samples were tested for $500 \mathrm{~h}$ in a Q-SUN Xe-3 Xenon arc lamp chamber. 


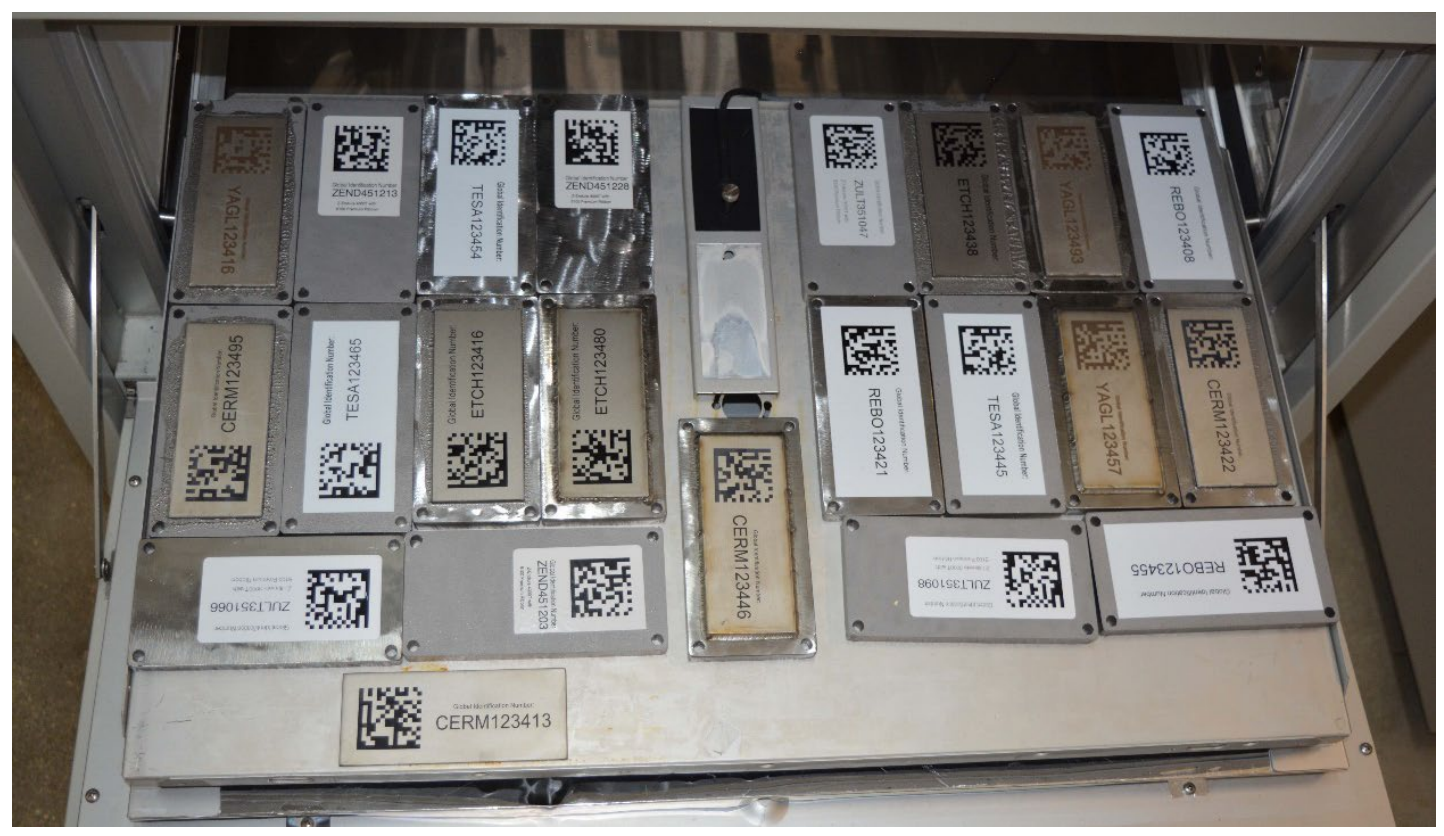

Figure 10. Barcode samples on Q-SUN Xe-3 Xenon arc lamp chamber loading tray.

\subsection{QUV UVA TESTING (TEST 2)}

Q-Lab staff installed 24 barcode samples into a QUV accelerated weathering chamber with UVA bulbs as shown in Figure 11 and Figure 12. This test replicated sunlight and dew weather conditions and followed a cycle like Cycle 1 in ASTM G154, "Standard Practice for Operating Fluorescent Ultraviolet (UV) lamp Apparatus for Exposure of Nonmetallic Materials." Samples were exposed to $8 \mathrm{~h}$ of light at $60^{\circ} \mathrm{C}$ black panel temperature followed by $4 \mathrm{~h}$ of condensation at $50^{\circ} \mathrm{C}$ black panel temperature. These cycles were repeated for a total of $500 \mathrm{~h}$. Fluorescent UVA-340 bulbs at $340 \mathrm{~nm}$ were used with an irradiance of $0.89 \mathrm{~W} /\left(\mathrm{m}^{2} \cdot \mathrm{nm}\right)$ [13]. A comprehensive list of samples subjected to QUV UVA testing is included in Appendix C. As a point of reference, Zebra suggests that $8 \mathrm{~h}$ of QUV exposure at $60^{\circ} \mathrm{C}$, followed by $4 \mathrm{~h}$ at $50^{\circ} \mathrm{C}$ for a total of $1,000 \mathrm{~h}$ is representative of about 1 year of exposure in southern Florida [14]. By this metric, our testing simulated about 6 months of total exposure in southern Florida. 


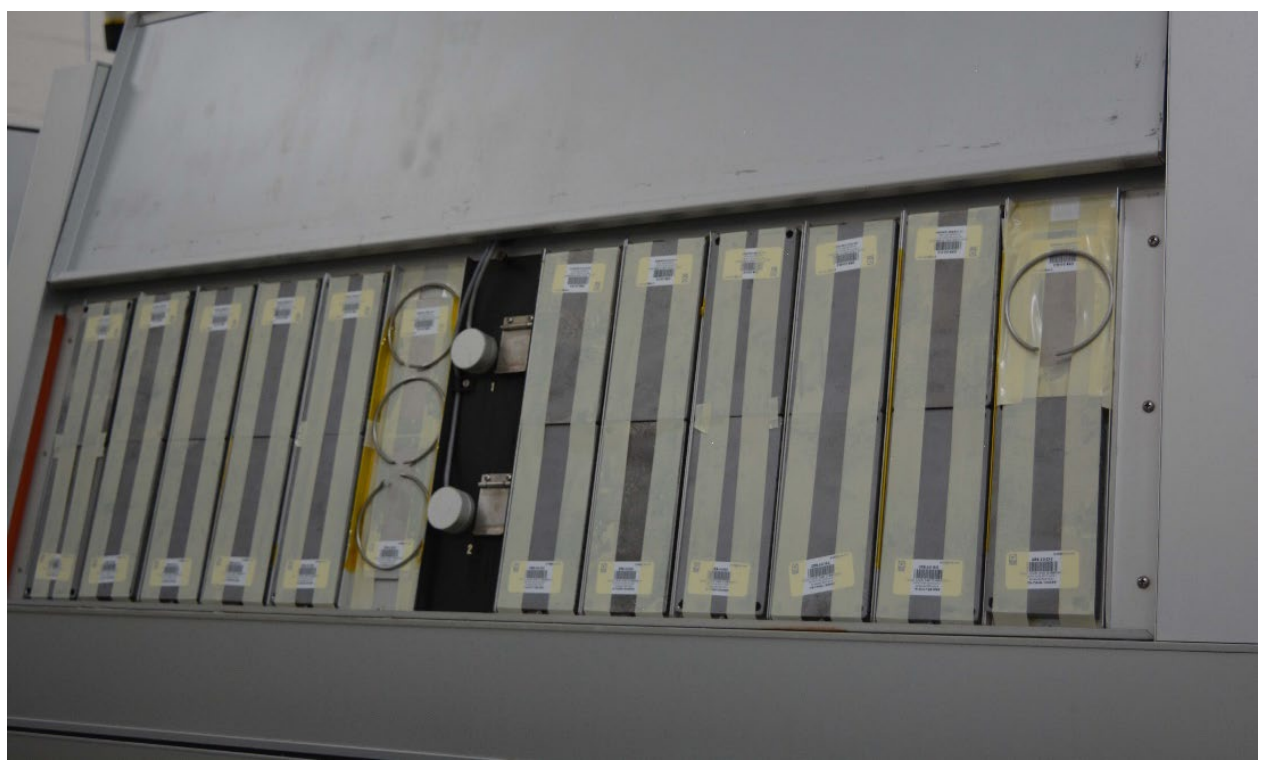

Figure 11. Barcode samples were initially UVA tested for $1000 \mathrm{~h}$ in a QUV accelerated weathering chamber.

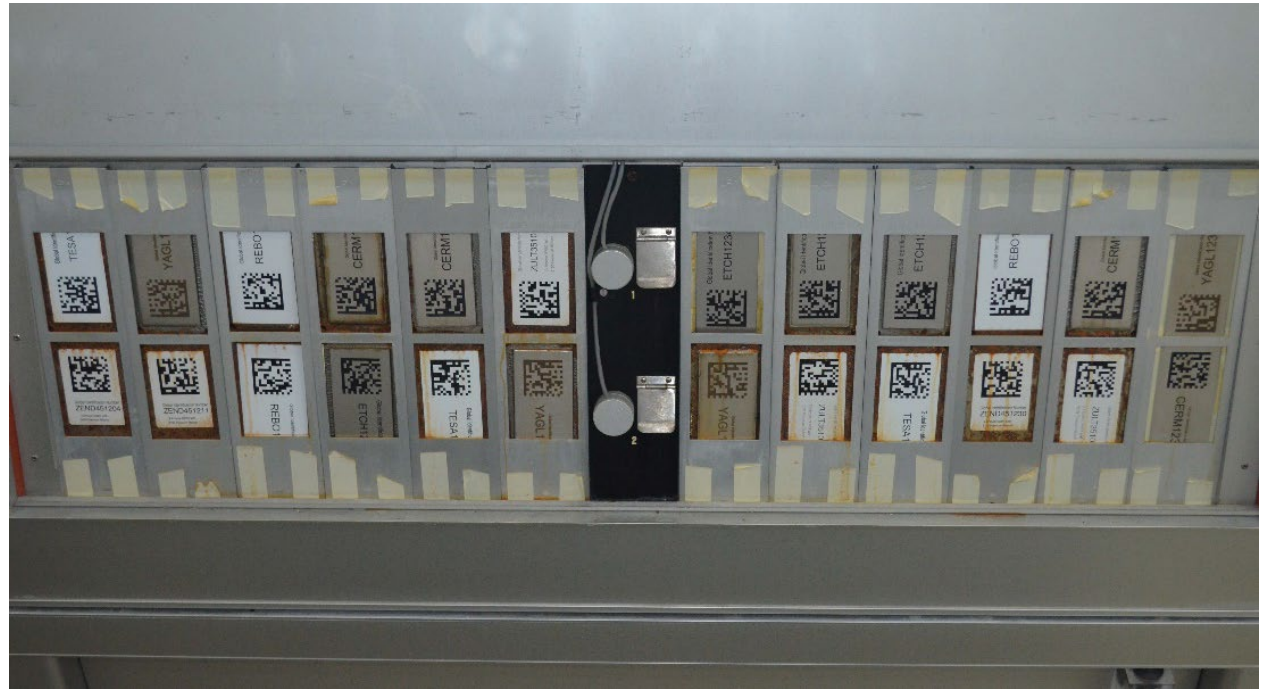

Figure 12. Barcode samples installed in QUV accelerated weathering chamber at test conclusion. Samples are shown facing out but were turned inward toward UV lamps during testing.QUV UVB TESTING (TEST 3)

Q-Lab staff installed 24 barcode samples into a QUV accelerated weathering chamber with UVB bulbs similar to the setup shown in Figure 11 and Figure 12. Testing followed a cycle like ASTM G154 Cycle 2. Samples were exposed to $4 \mathrm{~h}$ of light at $60^{\circ} \mathrm{C}$ black panel temperature followed by $4 \mathrm{~h}$ of condensation at $50^{\circ} \mathrm{C}$ black panel temperature. These cycles were repeated for a total of $500 \mathrm{~h}$. Fluorescent UVB-313 bulbs at $310 \mathrm{~nm}$ were used with an irradiance of $0.71 \mathrm{~W} /\left(\mathrm{m}^{2} \bullet \mathrm{nm}\right)$ [13]. A comprehensive list of samples subjected to QUV UVB testing is included in Appendix C.

\subsection{QUV AND Q-FOG TESTING (TEST 4)}

Q-Lab's staff exposed 24 barcode samples to a 4 week sequence of alternating cycles of 1 week in a QUV accelerated weathering chamber followed by 1 week in a Q-FOG cyclic corrosion (salt fog) chamber. Testing followed ASTM D5894, "Standard Practice for Cyclic Salt Fog/UV Exposure of Painted Metal, 
(Alternating Exposures in a Fog/Dry Cabinet and a UV/Condensation Cabinet)." In the QUV chamber, samples were set up similar to what is shown in Figure 11 and Figure 12. For 1 week $(168 \mathrm{~h})$, the samples were exposed to $4 \mathrm{~h}$ of light at $60^{\circ} \mathrm{C}$ black panel temperature followed by $4 \mathrm{~h}$ of condensation at $50^{\circ} \mathrm{C}$ black panel temperature. Fluorescent UVA-340 bulbs at $340 \mathrm{~nm}$ were used with an irradiance of 0.89 $\mathrm{W} /\left(\mathrm{m}^{2} \cdot \mathrm{nm}\right)$. At the end of 1 week, the samples were transferred to the salt fog chamber and set up as shown in Figure 13. The samples were exposed to $1 \mathrm{~h}$ of fog at ambient temperature then a $1 \mathrm{~h}$ dry off at $35^{\circ} \mathrm{C}$. The fog solution was $0.05 \%$ sodium chloride and $0.35 \%$ ammonium sulfate [15]. After 1 week (168 h) in the salt fog chamber, the samples were transferred back to the QUV accelerated weathering chamber, and the cycle was repeated. A comprehensive list of samples subjected to QUV and Q-FOG testing is included in Appendix C.

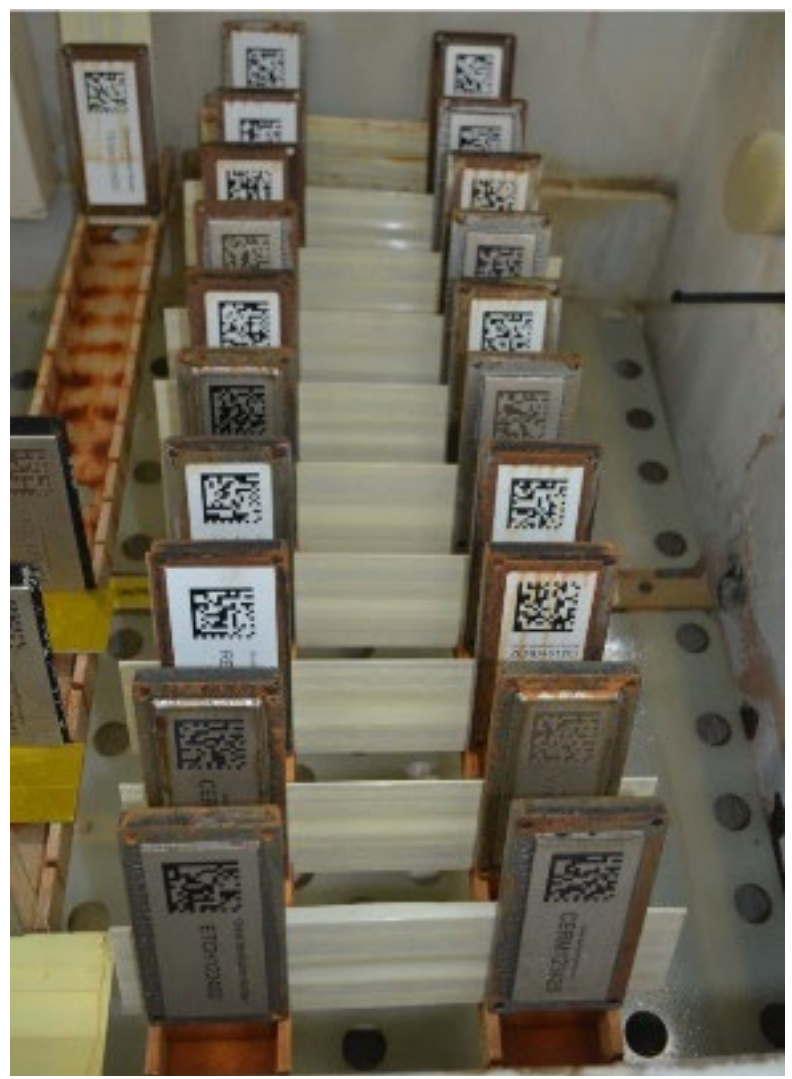

Figure 13. Barcode samples were tested for $168 \mathrm{~h}$ in a Q-FOG cyclic corrosion (salt fog) chamber.

\subsection{TEMPERATURE TESTING (TEST 5)}

ORNL staff leased a Tenney TC20RC environmental enclosure from Thermal Product Solutions and had it installed at ORNL. ORNL staff then installed 61 barcode samples as shown in Figure 14. All samples affixed to coupons were hung vertically. A few stainless-steel samples (CERM, ETCH, YAGL) that were not affixed to such coupons were laid flat on the stainless wire racks with their markings facing up. Samples were exposed to $-40^{\circ} \mathrm{C}$ for 7 days and then $113^{\circ} \mathrm{C}$ for 7 days. These cycles were repeated for a total of 6 weeks. Relative humidity was not controlled. The temperature ramp rate was not controlled such that the temperature changed as quickly as the chamber's heating and cooling capacity permitted. A comprehensive list of samples subjected to temperature testing is included in Appendix C. 


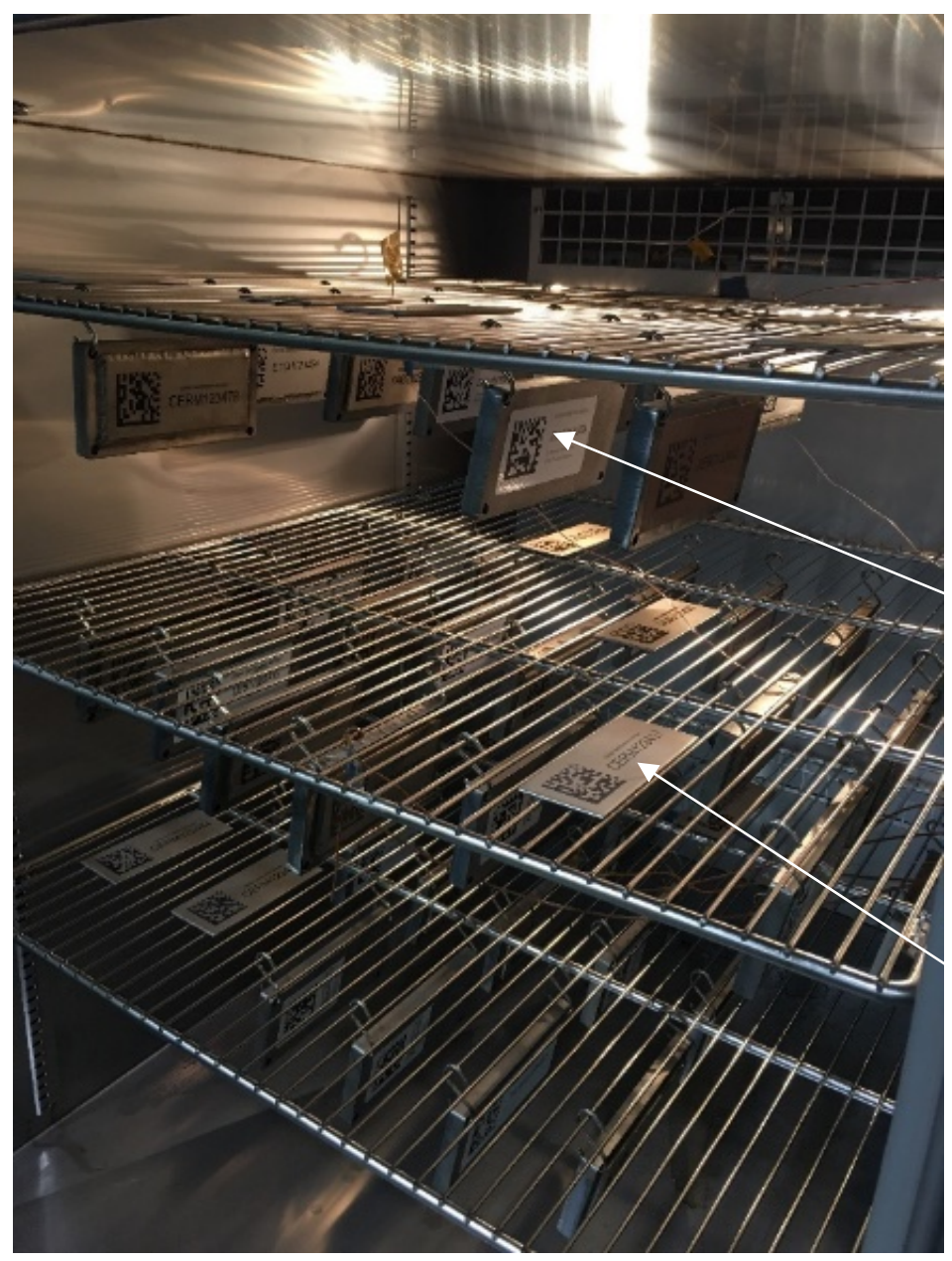

Hanging barcodes are attached to coupons

"Free" barcodes lay flat on the wire racks

Figure 14. Barcode samples were for 6 weeks in a Tenney TC20RC environmental enclosure.

\subsection{CYCLIC CORROSION TESTING (TEST 6)}

Staff at GTL installed 63 barcode samples into a salt fog chamber for cyclic corrosion testing. Samples were exposed to five cycles with each cycle consisting of the following steps:

1. Ambient stage with stress: $35 \pm 3^{\circ} \mathrm{C}$ chamber temperature, $48 \pm 3^{\circ} \mathrm{C}$ water temperature, approximately $95 \%$ relative humidity, and salt fog $(0.9 \%$ sodium chloride) for $8 \mathrm{~h}$

2. Humid stage: $49-60^{\circ} \mathrm{C}$ at approximately $95 \%$ relative humidity for $8 \mathrm{~h}$

3. Dry stage: $60^{\circ} \mathrm{C}$ at less than $30 \%$ relative humidity for $8 \mathrm{~h}$

One test cycle was equal to $24 \mathrm{~h}$. This testing is similar to that described by GMW 14872, "GM Cyclic Corrosion Laboratory Test - Issue 4" [16]. Barcode samples were loaded into the salt fog chamber (Figure 15) for the ambient and humid stages and then transferred to a Tenney C30RC environmental test chamber for the dry stage (Figure 16). A comprehensive list of samples subjected to cyclic corrosion testing is included in Appendix C. 


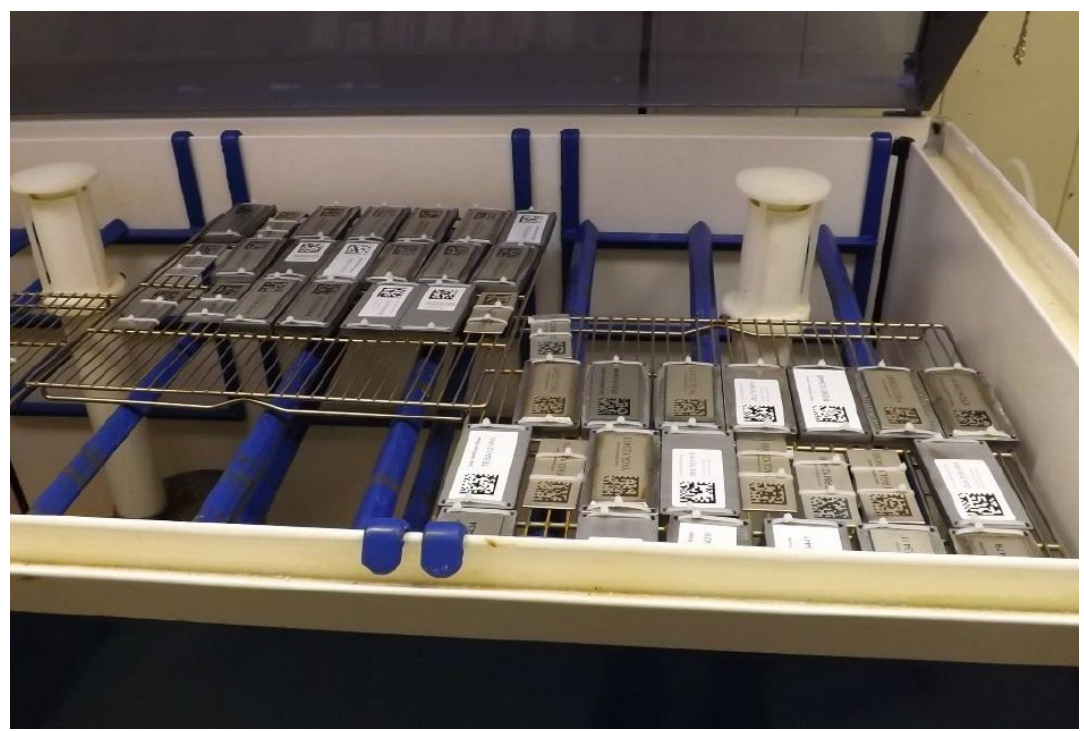

Figure 15. Barcode samples installed in GTL's salt fog chamber before ambient stage with stress exposure.
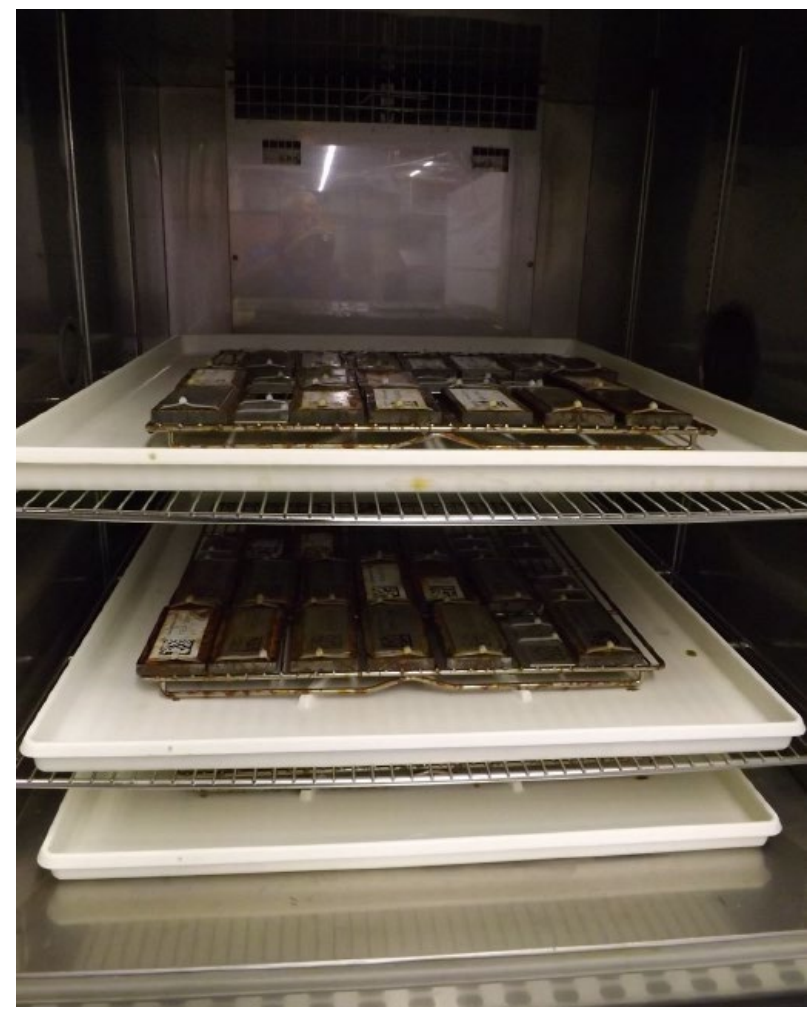

Figure 16. Barcode samples installed in GTL's environmental test chamber before dry stage exposure.

\subsection{CORROSION TESTING (TEST 7)}

Staff at GTL arranged 57 barcode samples in a salt fog chamber similar to the arrangement shown in Figure 15. Samples were exposed to a salt fog $(0.9 \%$ sodium chloride $)$ coupled with high temperature and high humidity for $200 \mathrm{~h}$. This test followed the cycle described in ASTM B117, "Standard Practice for Operating Salt Spray (Fog) Apparatus" [17]. The salt fog chamber had a temperature of $35 \pm 2{ }^{\circ} \mathrm{C}$, and the 
water temperature and relative humidity equalled $48 \pm 3^{\circ} \mathrm{C}$ and $95 \%$, respectively. A comprehensive list of samples subjected to corrosion testing is included in Appendix C.

\subsection{BLOWING SAND/DUST TESTING (TEST 8)}

Staff at GTL installed 57 barcode samples in a blowing sand/dust chamber (Figure 17). Testing followed MIL-STD 810G 510.6, "Sand and Dust 4.1 Procedure I - Blowing Dust," except temperature and humidity were not controlled [18]. Figure 18 shows how the dust was blown into the chamber. After testing, any dust that accumulated on the samples was brushed, wiped, or shaken off by methods that did not involve an air blast or vacuum cleaning. A comprehensive list of samples subjected to blowing sand/dust testing is included in Appendix C.

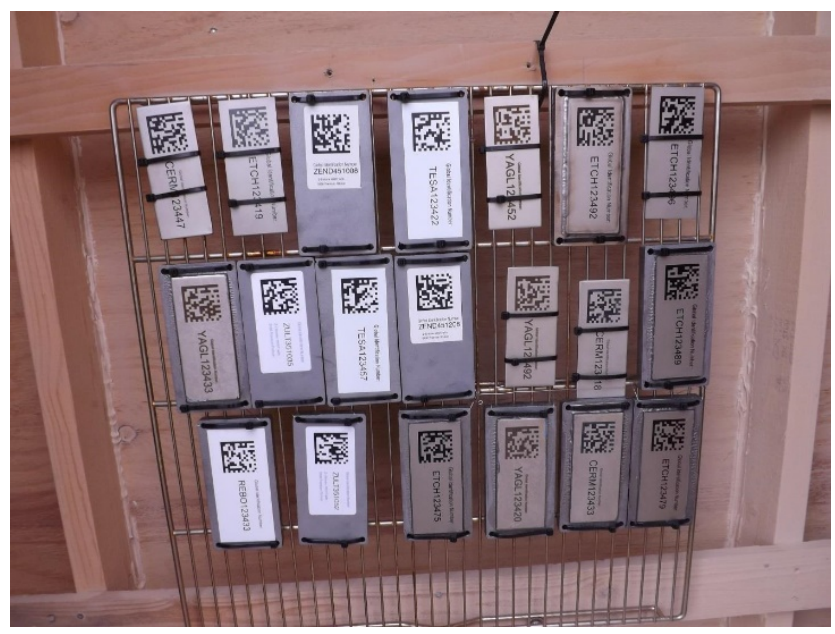

Figure 17. Barcode samples installed in GTL's sand/dust chamber before testing.

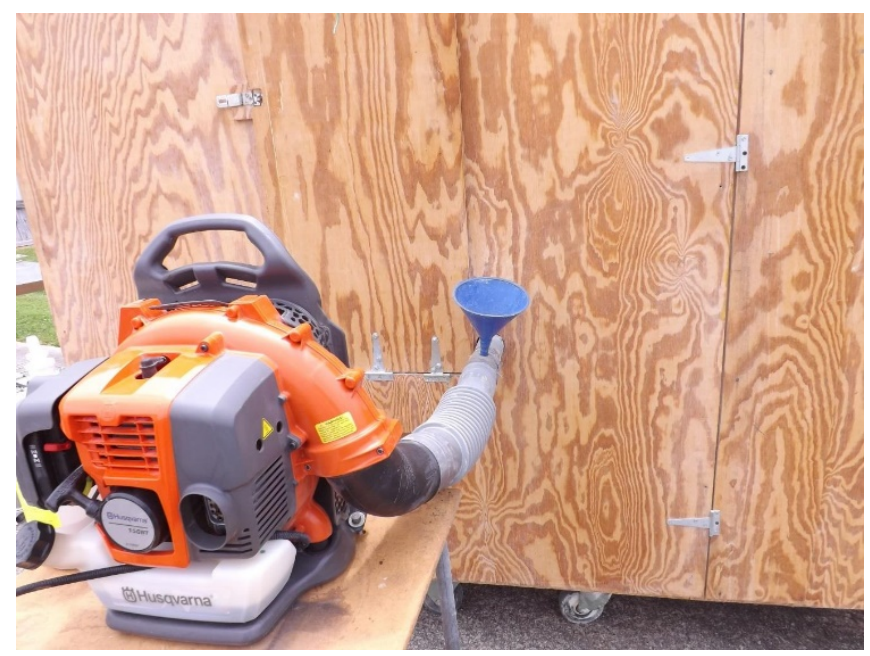

Figure 18. Introduction of test sand/dust into GTL's sand/dust chamber.

\subsection{IMPACT TESTING (TEST 9)}

Staff at GTL completed impact testing of 55 barcode samples. Testing followed IEC 61010-1, Section 8.2.2, "Impact Test" [19]. A smooth steel sphere with a mass of $500 \mathrm{~g}$ was placed 1,000 $\mathrm{mm}$ above the center of the barcode sample inside a guide tube (Figure 19). The removal of the pin (Figure 19, left) allowed the sphere to freely fall onto the sample for impact testing. Figure 20 shows the sphere on the 
YAGL123469 barcode sample after the guide tube was removed postimpact testing. A comprehensive list of samples subjected to impact testing is included in Appendix C.
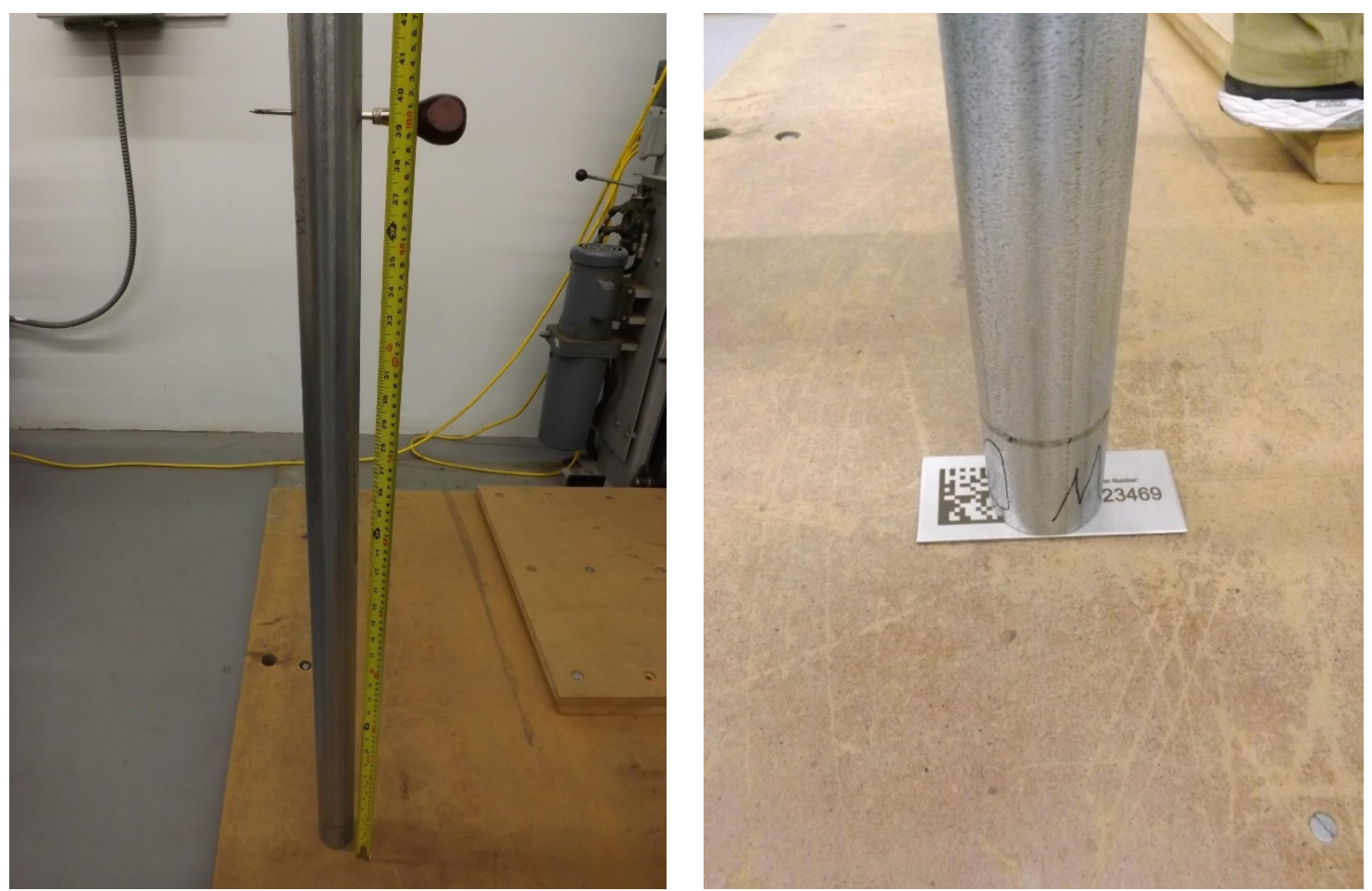

Figure 19. A $500 \mathrm{~g}$ smooth steel sphere is placed inside the guide tube $1,000 \mathrm{~mm}$ above the barcode sample surface. When the pin is removed, the sphere falls freely onto the sample for impact testing.

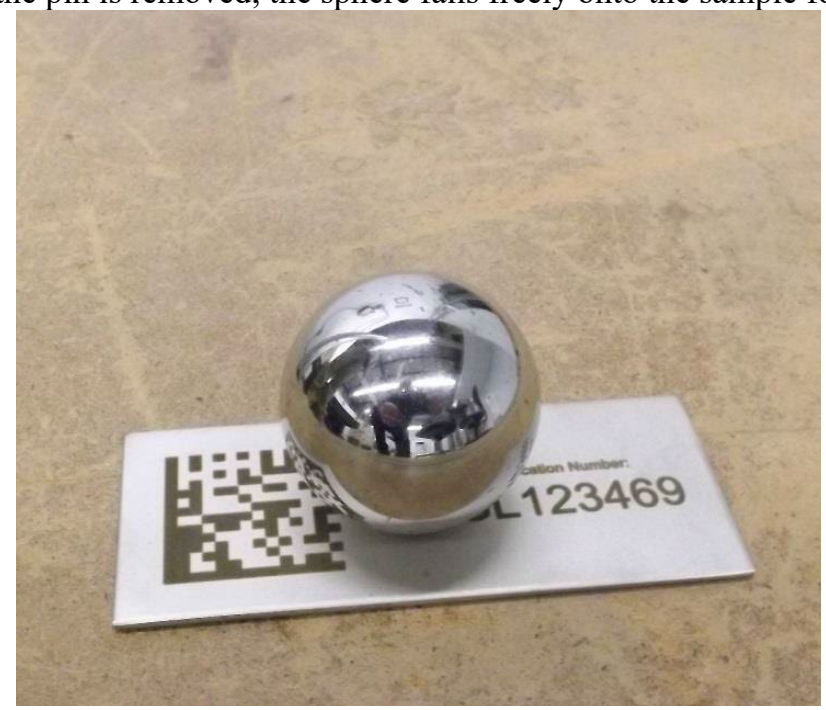

Figure 20. A $500 \mathrm{~g}$ smooth steel sphere on the YAGL123469 barcode sample after impact testing.

\subsection{HIGH PRESSURE AND TEMPERATURE WATER JET TESTING (TEST 10)}

Staff at GTL subjected 57 barcode samples to high-pressure and high-temperature water jet testing. Testing followed IEC 60529 CORR 1 - Degrees of Protection Provided by Enclosures (IP Code) - Edition 2.2, Test 14.2.9 "Test for second characteristic numeral 9 with a spray nozzle" [20]. The samples were attached to a grate using zip ties and then subsequently sprayed with a stream of water generated by a 
standard test nozzle (Figure 21). A comprehensive list of samples subjected to high pressure and temperature water jet is included in Appendix C.

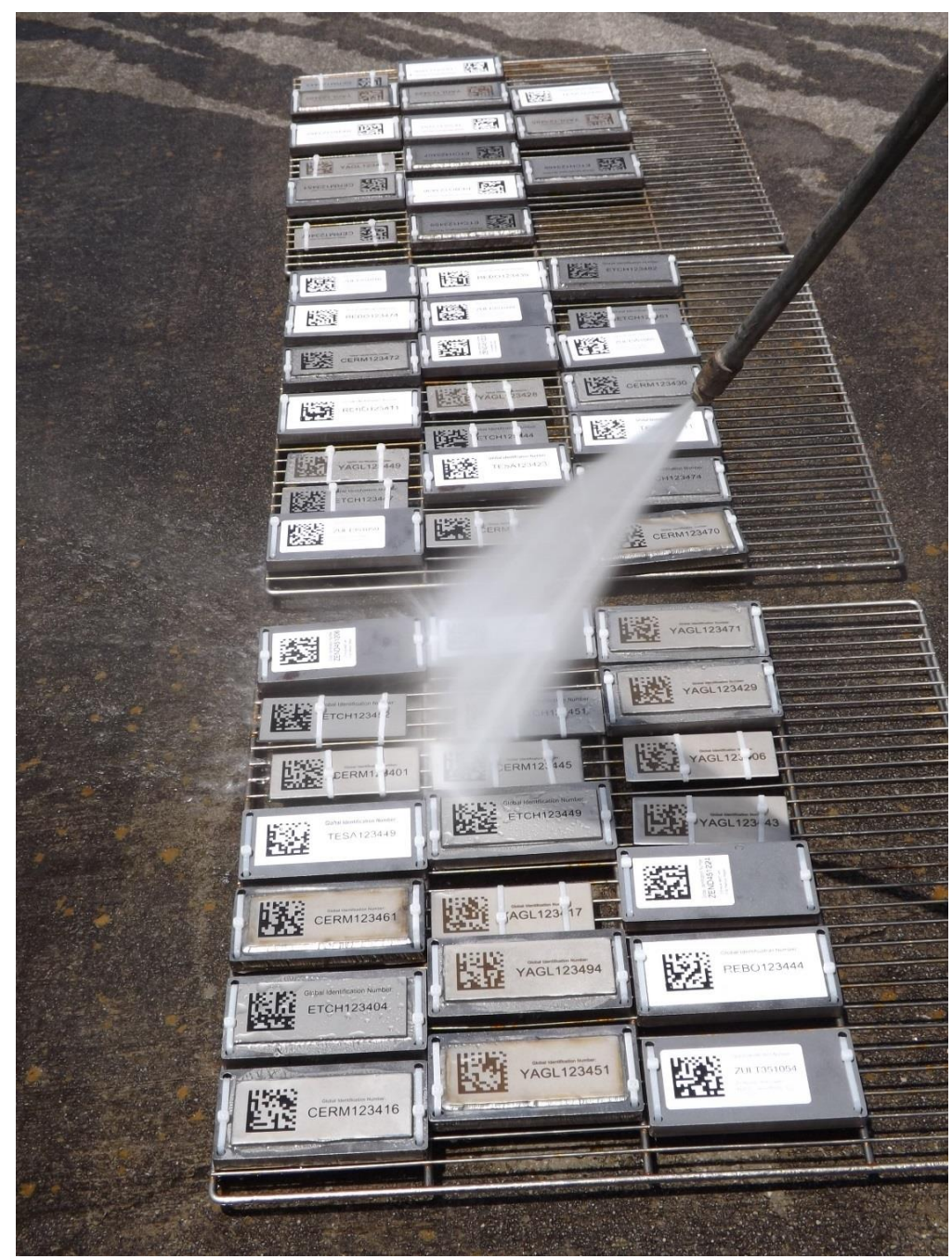

Figure 21. Barcode samples subjected to high pressure and temperature water jet testing.

\section{RESULTS BY ENVIRONMENTAL TEST}

The ORNL team made both qualitative and quantitative assessments to evaluate the impact of the various environmental tests on the barcode samples. The test plan called for each batch of samples to be photographed and scanned using the barcode verifier before and after the environmental tests. Qualitative results were collected by comparing the photographs of the barcode samples before and after environmental exposure; quantitative results were collected by comparing the symbol contrast (ISO 15415) or cell contrast (ISO 29158) values from the barcode verifier scans before and after exposure. This Section provides the results from each of the ten environment tests. 


\subsection{Q-SUN XENON ARC LAMP TESTING (TEST 1)}

\subsubsection{Qualitative}

Figure 22 shows the 22 barcode samples before (left) and after (right) exposure to the Q-SUN xenon arc lamp testing. As shown in the figures, a mild discoloration is visible on the coupons after the exposure. Discoloration is also present on the stainless-steel samples (CERM, ETCH, and YAGL); however, most of the label-type barcode samples (REBO, TESA, ZEND, and ZULT) appeared unaffected. There does not appear to be any pattern to or correlation between the discoloration of a sample and its respective position within the test environment.
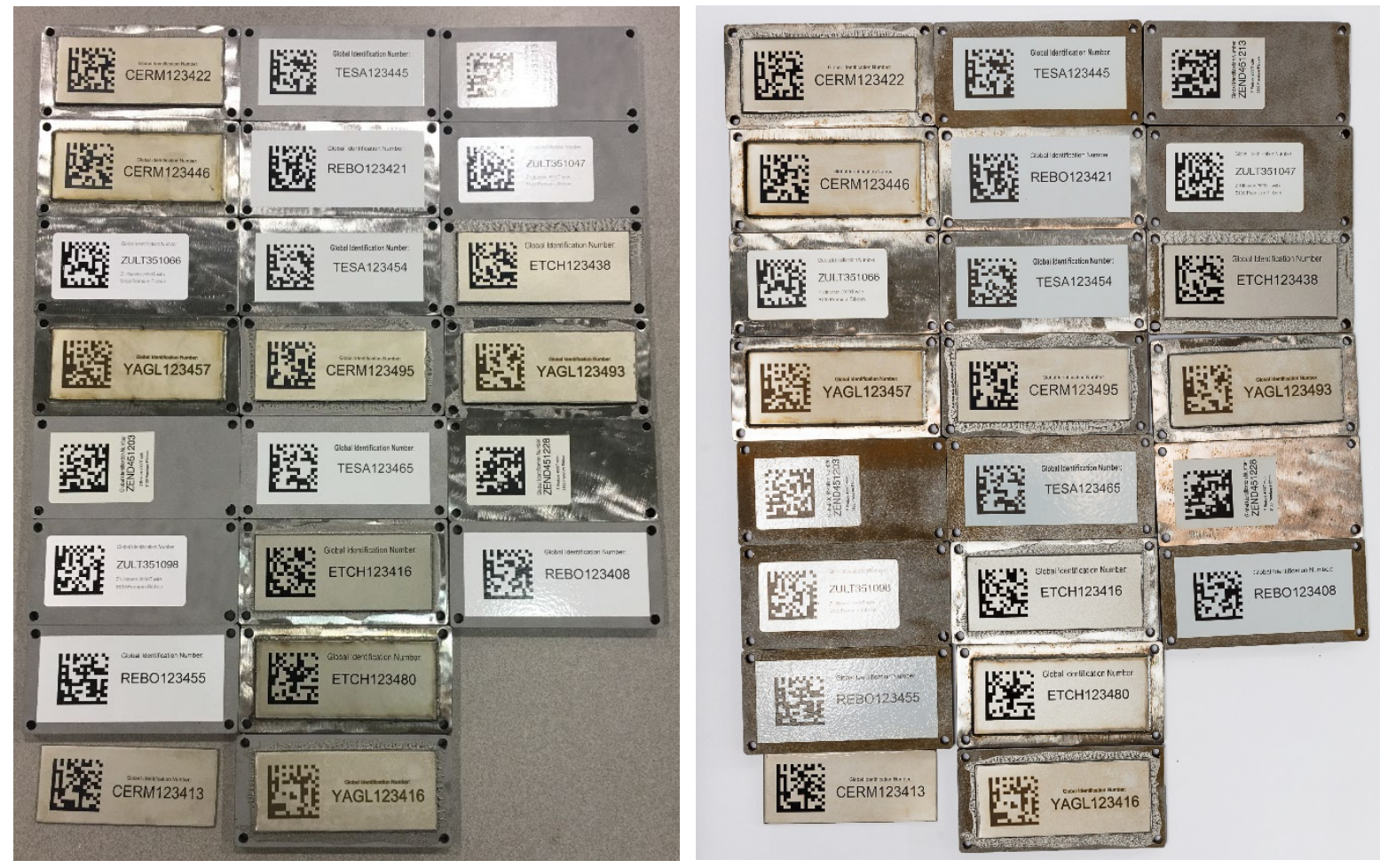

Figure 22. Barcode samples before (left) and after (right) Q-SUN xenon arc lamp testing. 


\subsubsection{Quantitative}

After exposure, all 22 samples were successfully decoded with the barcode verifier. Figure 23 shows the cell or symbol contrast metric, depending on the appropriate standard, before and after exposure for samples subjected to Q-SUN xenon arc lamp testing. The ETCH stainless-steel samples showed the largest decrease in cell contrast compared to all other sample types; however, this decrease was not significant enough to prevent barcode decoding for any sample group.

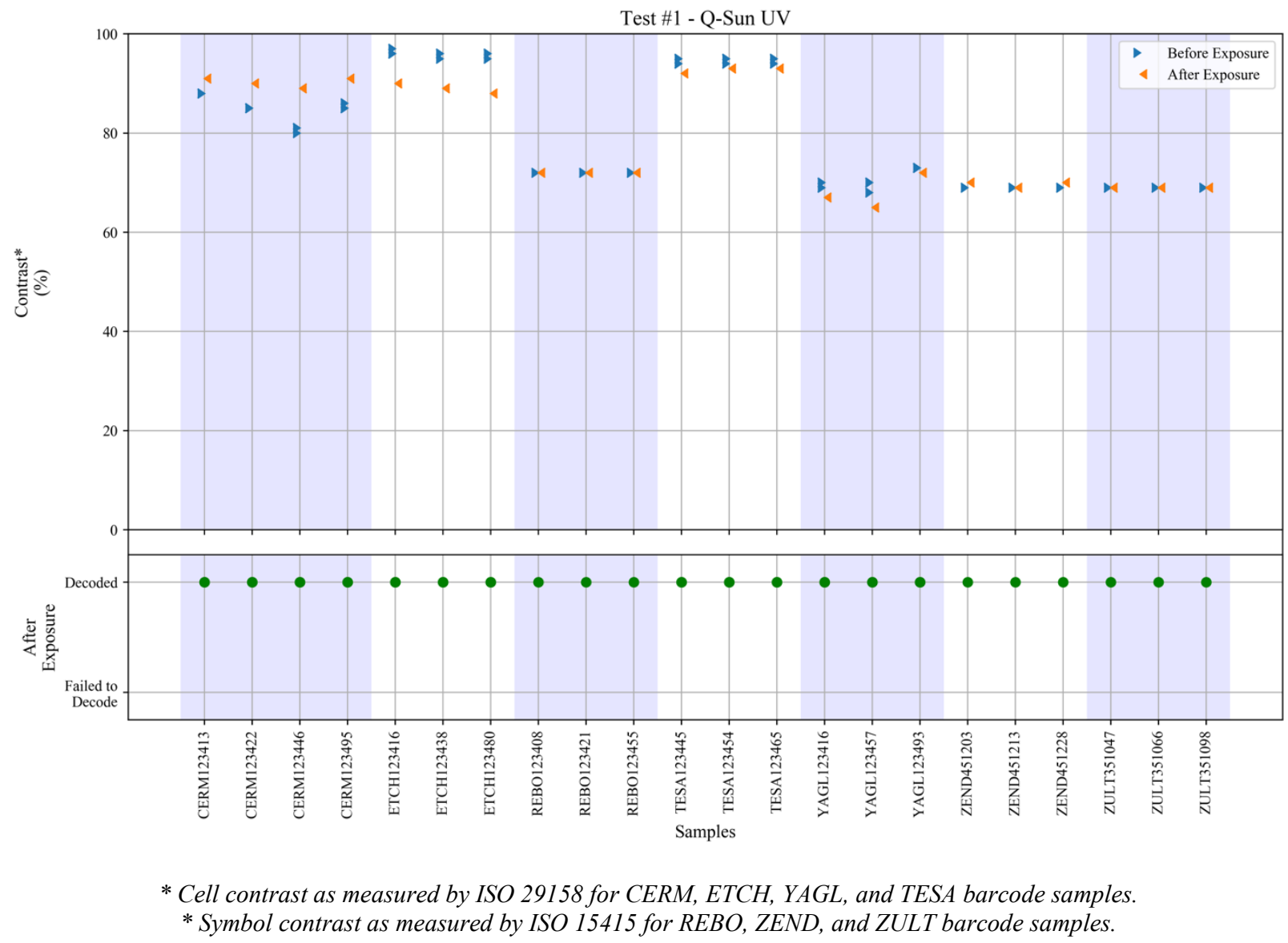

Figure 23. Cell or symbol contrast for barcode samples before and after Q-SUN xenon arc lamp testing. 


\subsection{QUV UVA TESTING (TEST 2)}

\subsubsection{Qualitative}

Figure 24 shows the 24 barcode samples before (left) and after (right) exposure to QUV UVA testing. The A516 coupons showed moderate signs of rust after exposure. The stainless-steel and label-type barcode samples themselves also appeared to have a rusty surface residue. It is believed that the A516 coupons rusted as a result of the condensation portion of the test cycle and moisture transported rust from the A516 coupon onto the barcode samples.
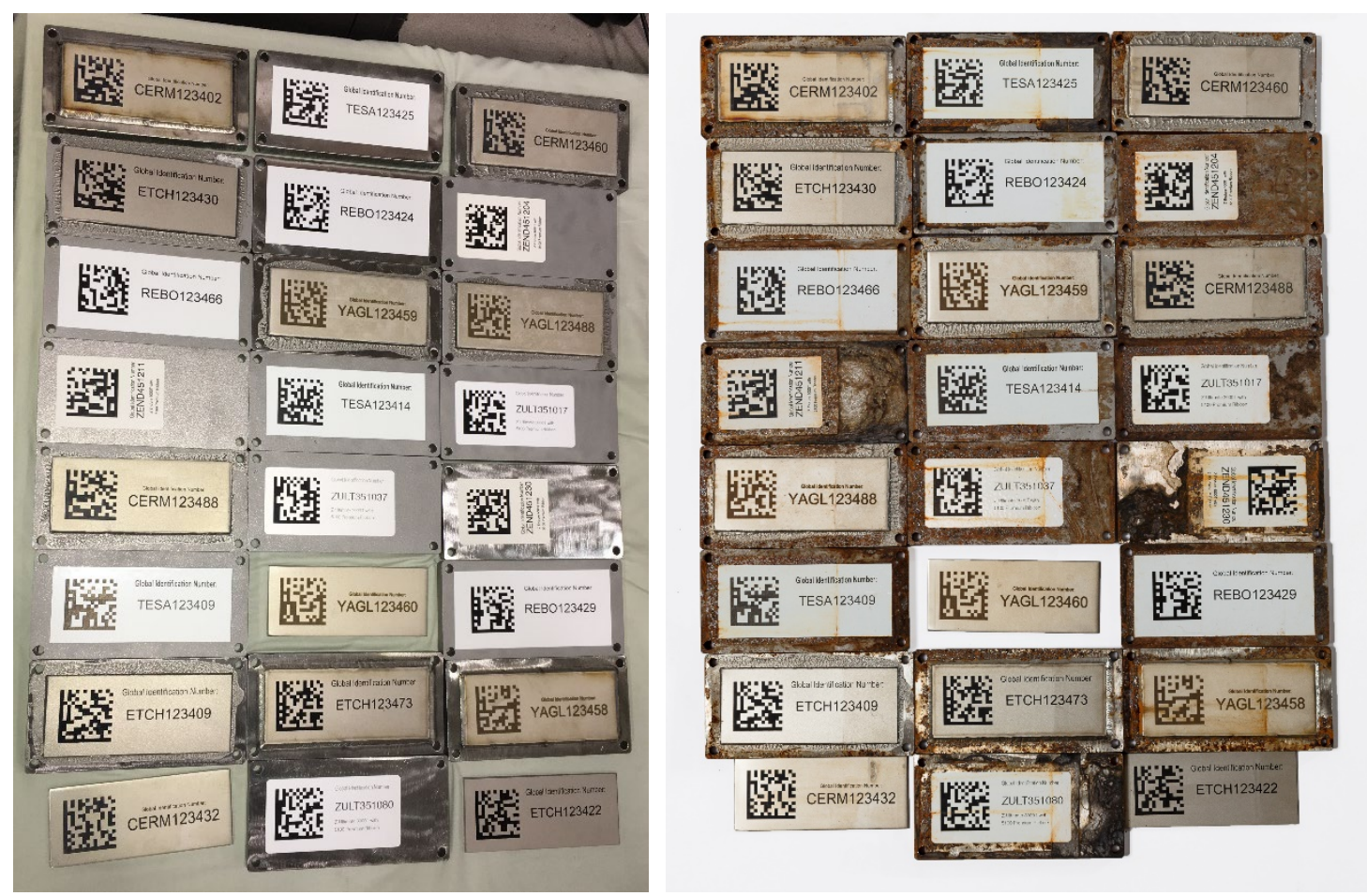

Figure 24. Barcode samples before (left) and after (right) QUV UVA testing. 


\subsubsection{Quantitative}

After exposure, all 24 samples were successfully decoded with the barcode verifier. Figure 25 shows the cell or symbol contrast metric before and after exposure for samples subjected to UVA testing. Similar to Test 1, a majority of these samples showed little or no decrease in cell or symbol contrast from UVA exposure. The ETCH stainless-steel samples showed a larger decrease in cell contrast than the other sample types; however, this decrease was not significant enough to prevent barcode decoding for any sample group.

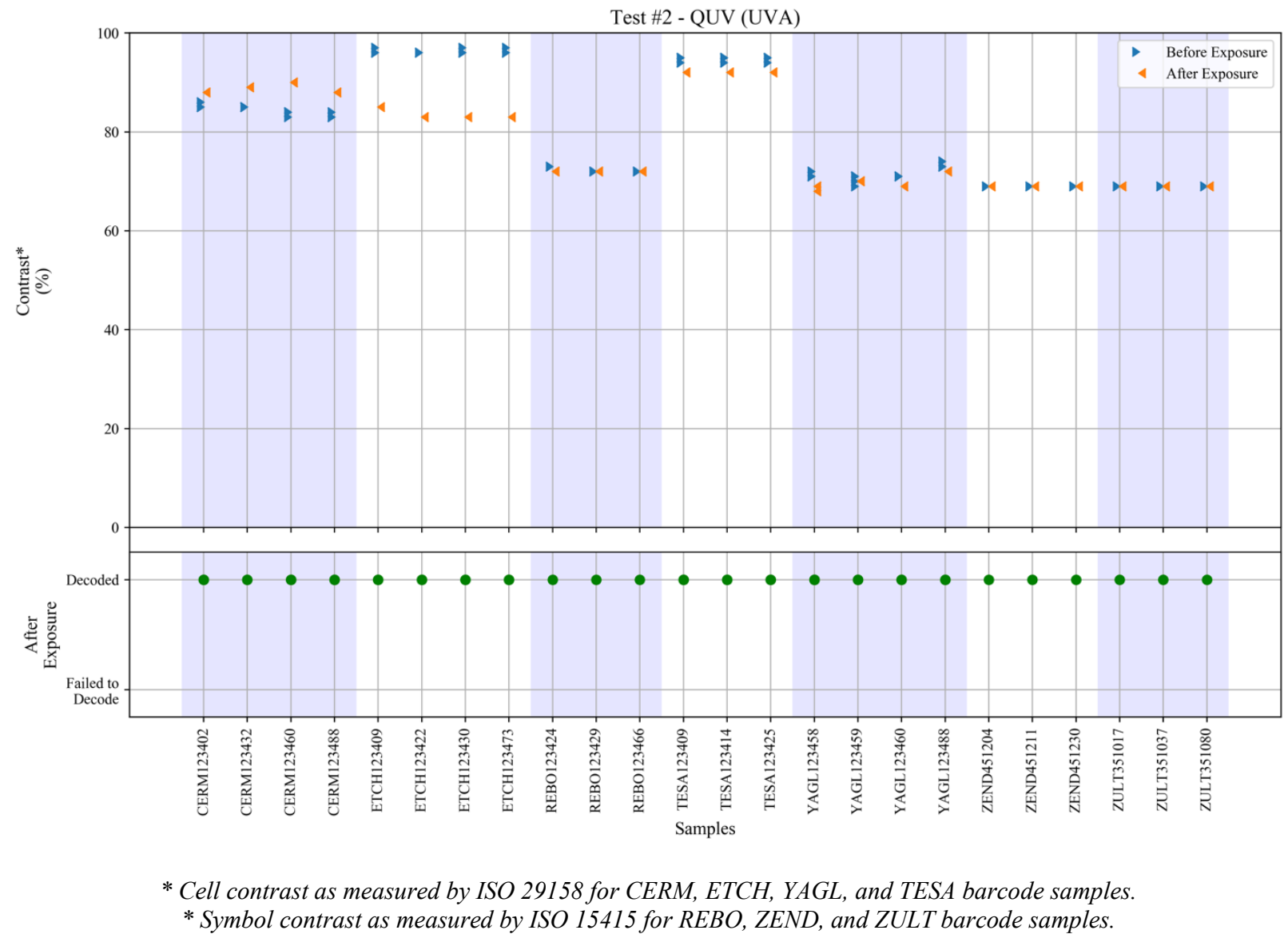

Figure 25. Cell or symbol contrast for barcode samples before and after QUV UVA testing. 


\subsection{QUV UVB TESTING (TEST 3)}

\subsubsection{Qualitative}

Figure 26 shows the 24 barcode samples before (left) and after (right) exposure to QUV UVB testing. The A516 coupons showed moderate signs of corrosion after exposure. The stainless-steel and label-type barcode samples themselves also appeared to have a rusty surface residue. We believe the A516 coupons rusted as a result of the condensation portion of the test cycle and moisture transported rust from the A516 coupon onto the barcode samples.
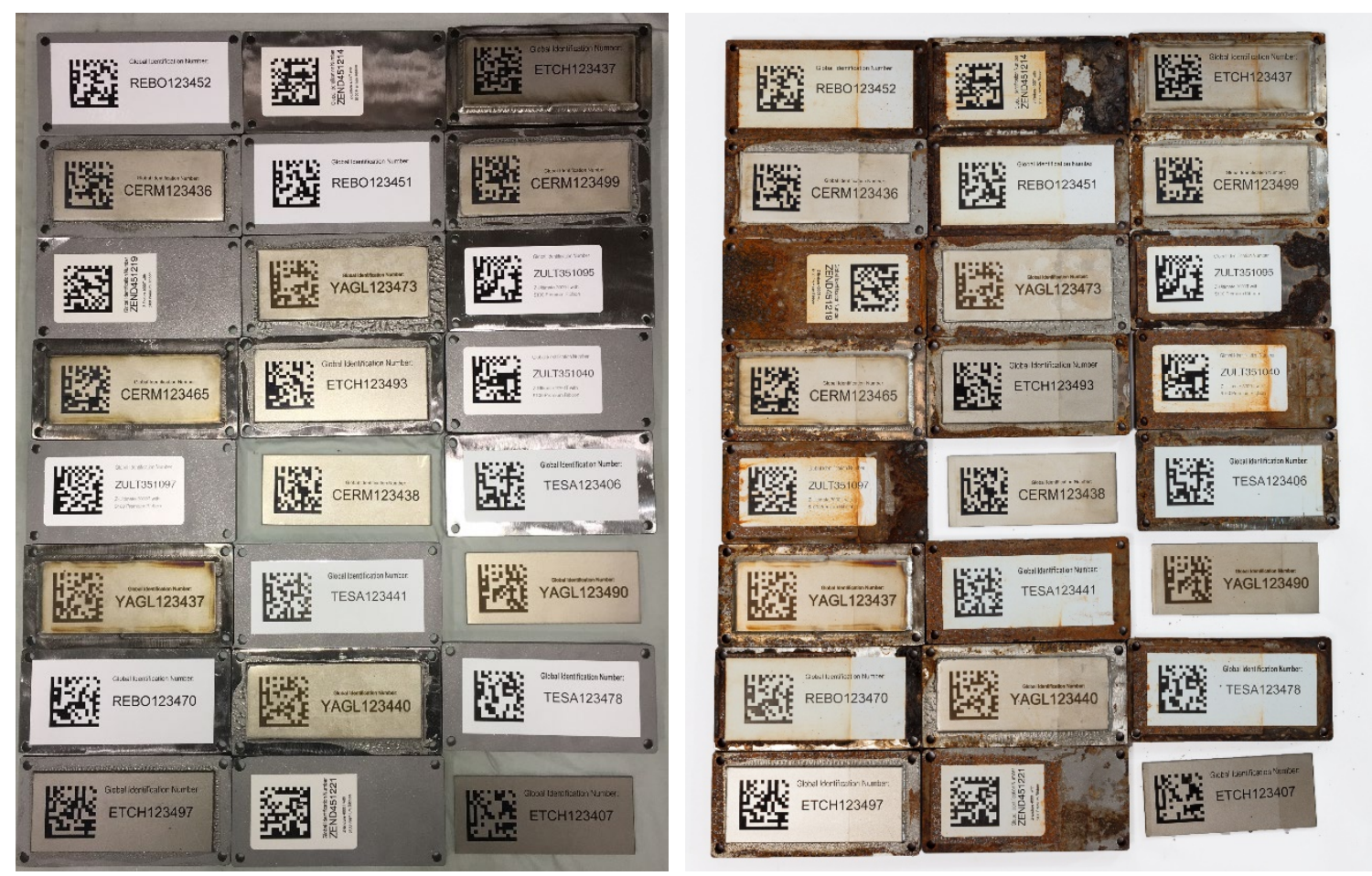

Figure 26. Barcode samples before (left) and after (right) UVB testing. 


\subsubsection{Quantitative}

After exposure, all 24 samples were successfully decoded with the barcode verifier. Figure 27 shows the cell or symbol contrast metric before and after exposure for samples subjected to UVB testing. Similar to Test 1 and Test 2, a majority of these samples showed little or no degradation following this test.

Consistent with other UV tests, the ETCH stainless-steel samples showed a larger decrease in cell contrast compared to the other barcode samples. Exposure from this test did not prevent barcode identification for any sample group.

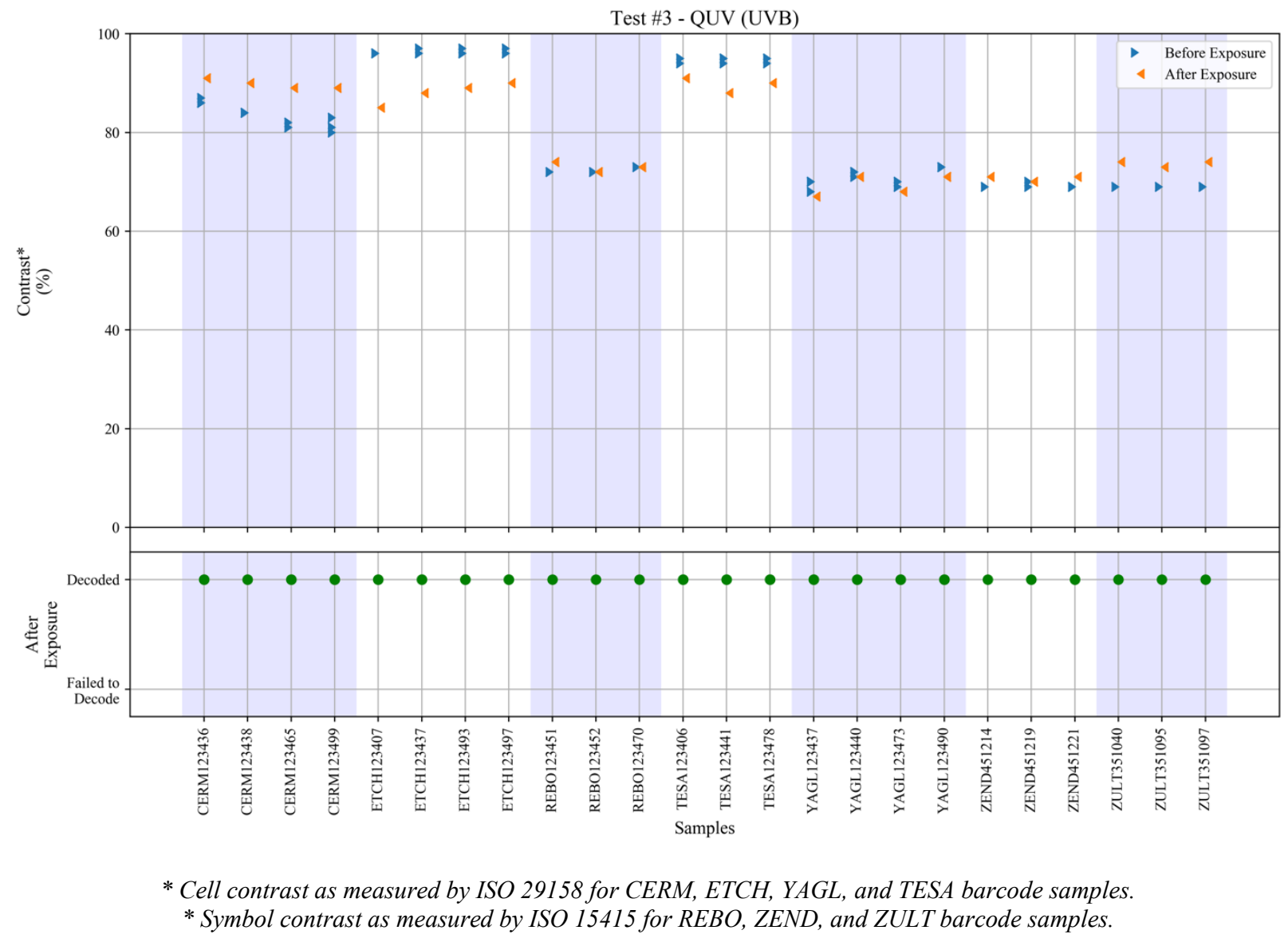

Figure 27. Cell or symbol contrast for barcode samples before and after QUV UVB testing. 


\subsection{QUV AND Q-FOG TESTING (TEST 4)}

\subsubsection{Qualitative}

Figure 28 shows the 24 barcode samples before (left) and after (right) exposure to QUV and salt fog testing. The A516 coupons experienced significant rust. Many of the stainless-steel samples and especially the label-type samples also appeared to have a rusty surface residue.
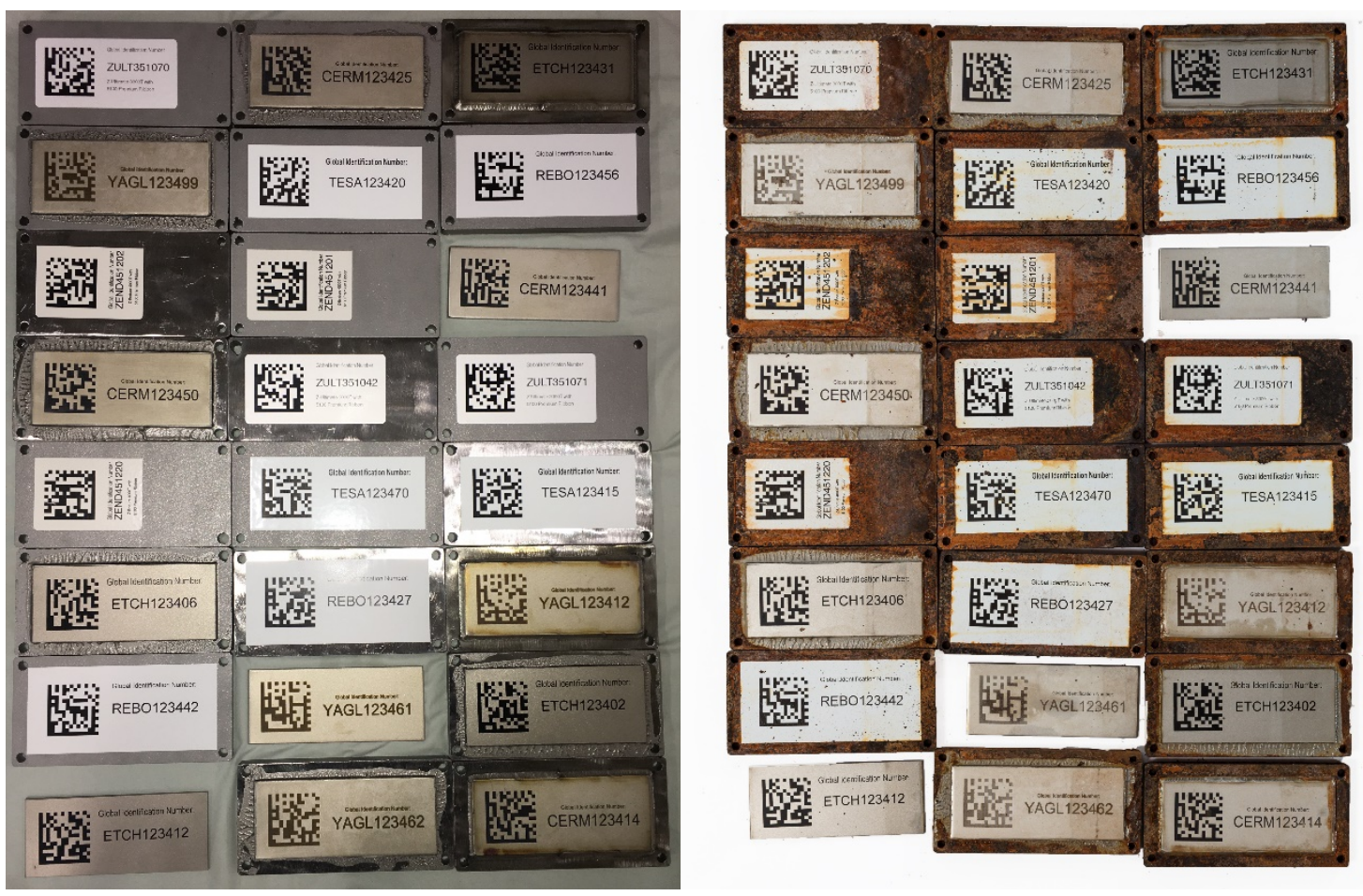

Figure 28. Barcode samples before (left) and after (right) QUV and Q-FOG testing.

It is believed that the A516 coupons rusted as a result of the Q-FOG portion of the test cycle and moisture transported rust from the top down the front face of the samples. As shown in Figure 29, the specimens were installed in the Q-FOG machine with the barcode at the top. Figure 30, showing ZEND451202 with rust colored stains down the front face, is consistent with our hypothesis that moisture transported rust from the exposed A516 surface above the sample onto the sample surface. The label-type samples appear to have more rusty surface residue than the stainless-steel samples, which is likely because the thickness of the stainless-steel samples diverted moisture to the side and around the sample instead of over the front face of the thin label-type samples. 


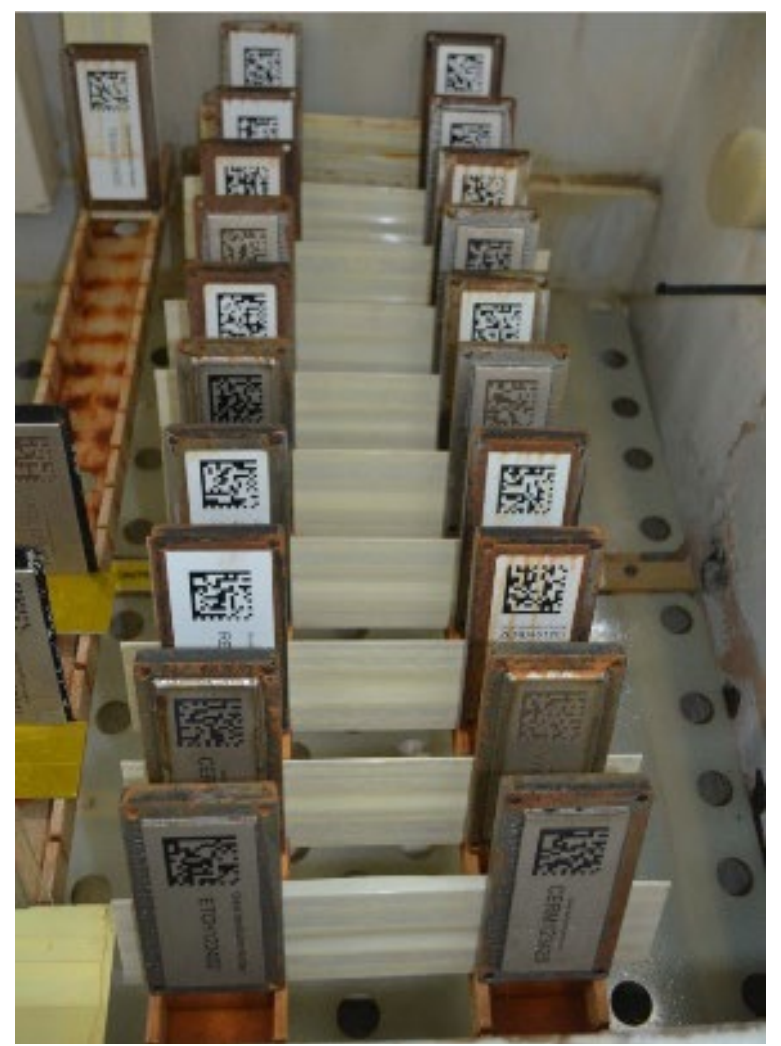

Figure 29. Barcode samples installed in Q-FOG cyclic corrosion (salt fog) chamber.

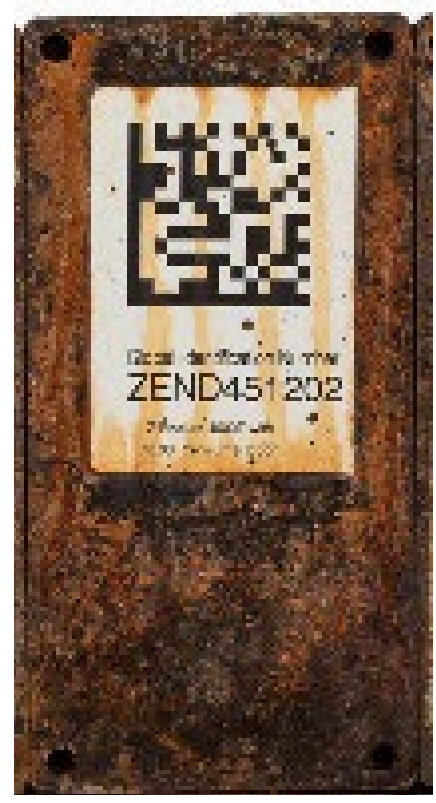

Figure 30. ZEND451202 with rust colored stains.

As described in the next section, one or more barcodes failed to decode after exposure. The authors suspected the barcodes were failing to decode because of an excessive amount of rusty residue on the samples. As a result each barcode sample was hand cleaned. Sample cleaning included a simple process with readily available supplies. Formula 409 cleaner was applied to each sample then wiped dry with a 
Pig PR40 all-purpose wipe. The process was repeated with WD-40 solution. This cleaning process was not applied to the coupons. Figure 31 shows the samples after hand cleaning; all samples were then scanned again with the barcode verifier. The cleaning process was qualitatively very effective for all sample types except ZEND. As shown in Figure 32, the rusty residue seemed to permanently stain the ZEND labels.

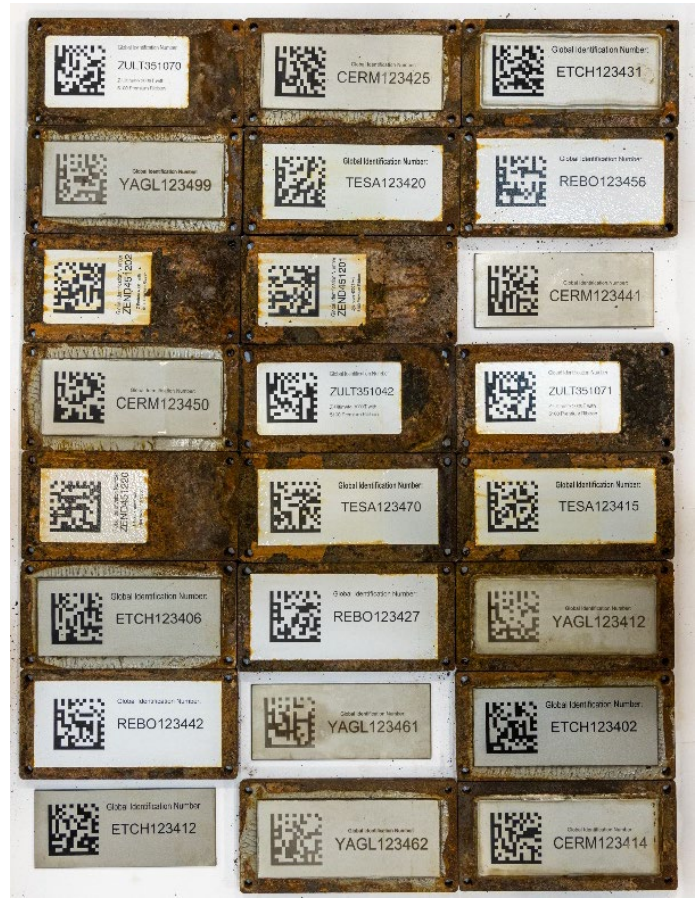

Figure 31. Barcode samples subjected to QUV and Q-FOG testing after hand cleaning.
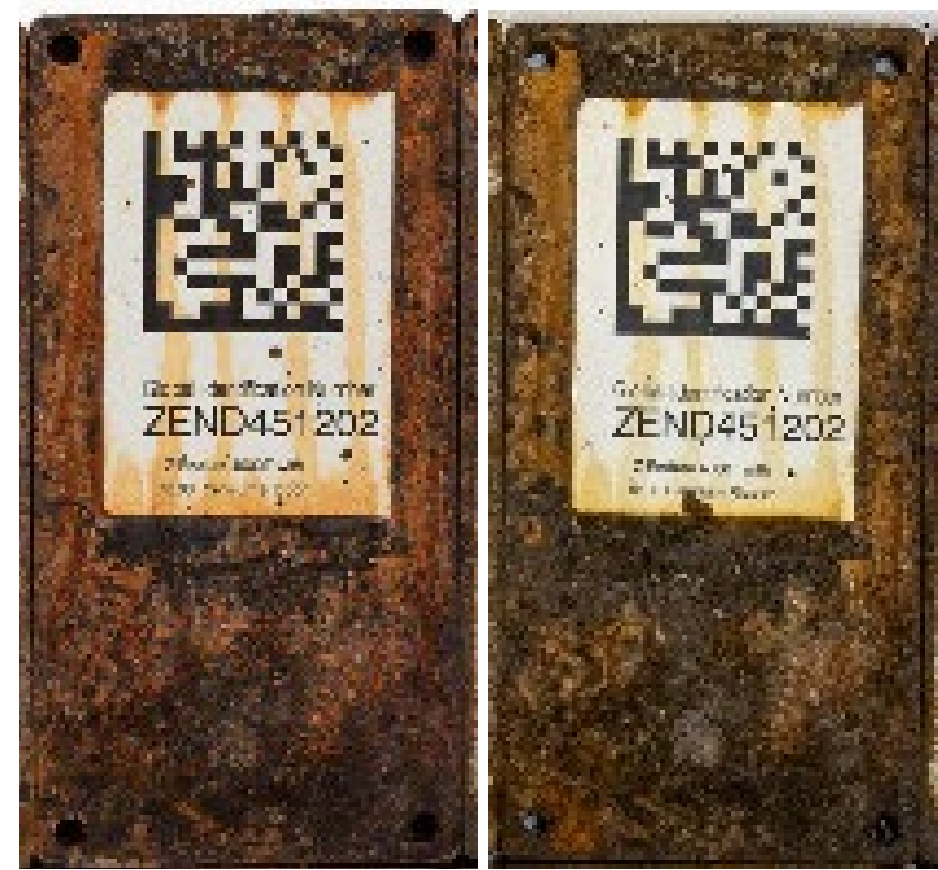

Figure 32. ZEND451202 before (left) and after (right) cleaning. 


\subsubsection{Quantitative}

All samples were scanned after exposure. After exposure, 23 of the 24 samples were successfully decoded with the barcode verifier (YAGL123462 was not decoded). When compared with the rest of the tested samples, it is unclear why this sample did not initially decode. After the set of samples was hand cleaned with the method described above, all 24 samples successfully decoded, including YAGL123462. Figure 33 shows the cell or symbol contrast metric before exposure, after exposure, and after cleaning for samples subjected to UV and salt fog testing.

The contrast of the stainless-steel samples changed noticeably after exposure:

- CERM: contrast increased after exposure and increased slightly after cleaning

- ETCH: contrast decreased after exposure and increased back to pre-exposure level after cleaning

- YAGL: contrast decreased after exposure and tended to increase after cleaning

The label-type samples (REBO, TESA, ZEND, and ZULT) experienced little or no decrease in cell or symbol contrast as a result of exposure. Sample cleaning did not significantly change the contrast for the label-type samples.

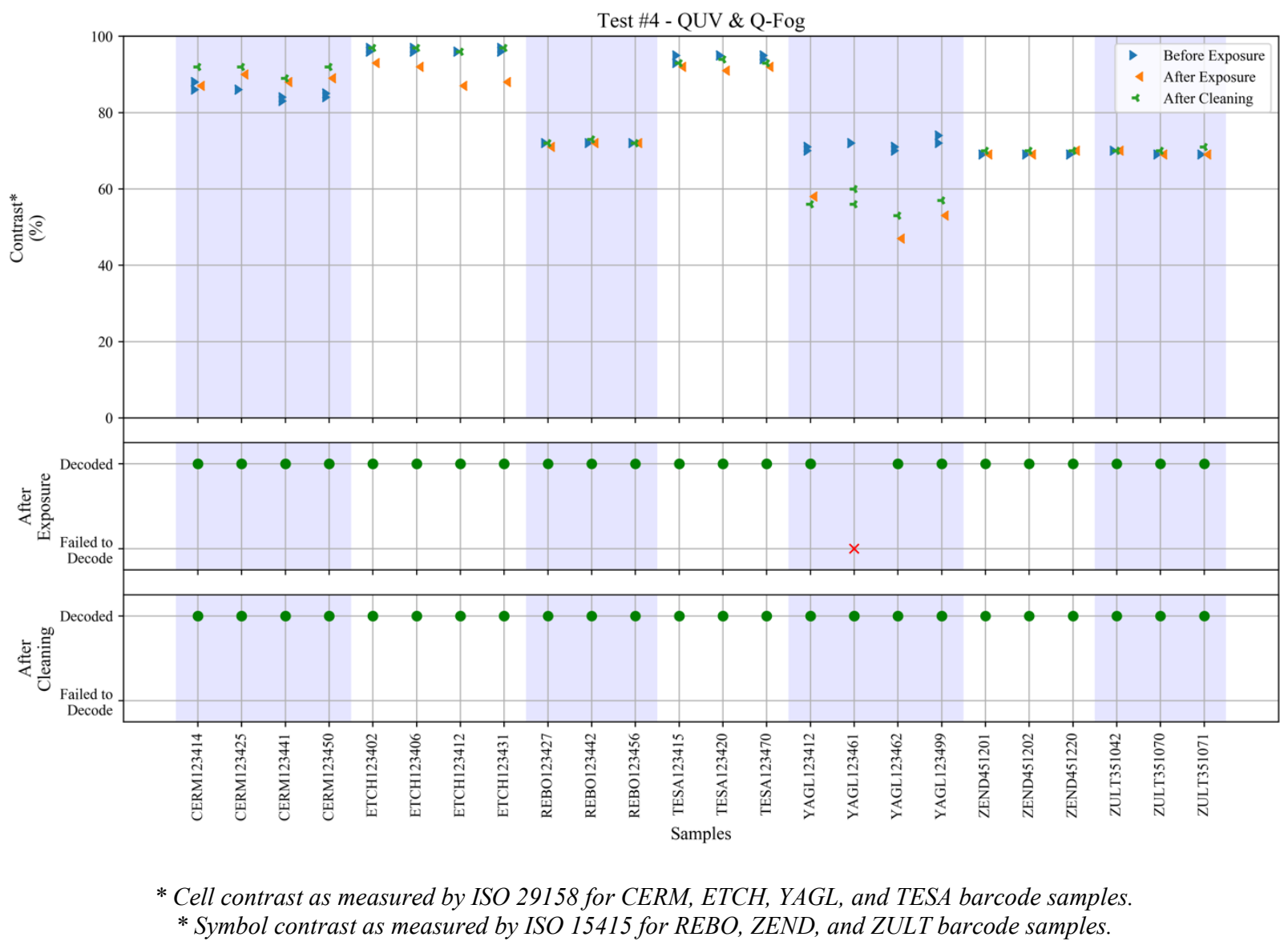

Figure 33. Before exposure, after exposure, and after cleaning cell or symbol contrast for barcode samples subjected to QUV and Q-FOG testing. 


\subsection{TEMPERATURE TESTING (TEST 5)}

\subsubsection{Qualitative}

Figure 34 shows the 61 barcode samples before (top) and after (bottom) exposure to temperature testing. As shown in Figure 35, the REBO samples seemed to discolor as a result of exposure.

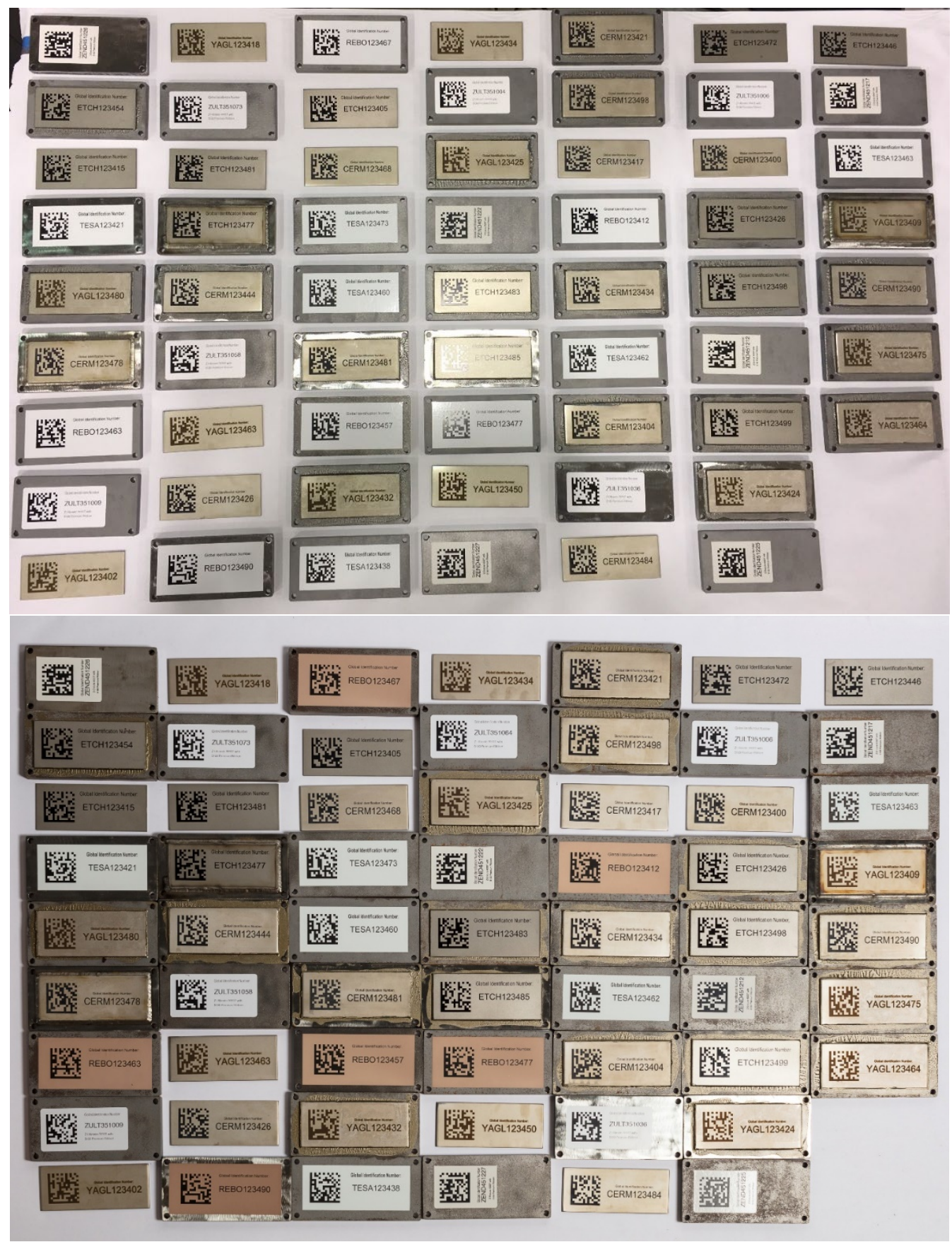

Figure 34. Barcode samples before (top) and after (bottom) temperature testing. 

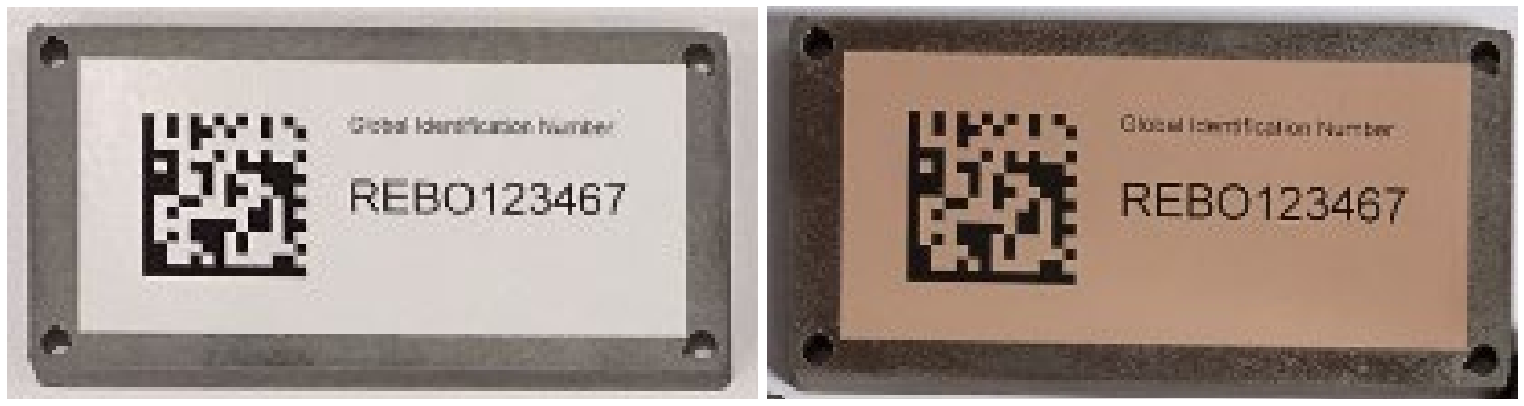

Figure 35. REBO123467 before (left) and after (right) temperature testing.

\subsubsection{Quantitative}

After exposure, all 61 samples were successfully decoded by the barcode verifier. Figure 36 shows the cell or symbol contrast metric before and after exposure for samples subjected to cyclic temperature testing. For the majority of the samples, a significant decrease in cell or symbol contrast did not occur as a result of exposure. However, the REBO samples showed a larger decrease in symbol contrast than the rest of the samples. As noted, this decrease did not preclude decoding by the barcode verifier.

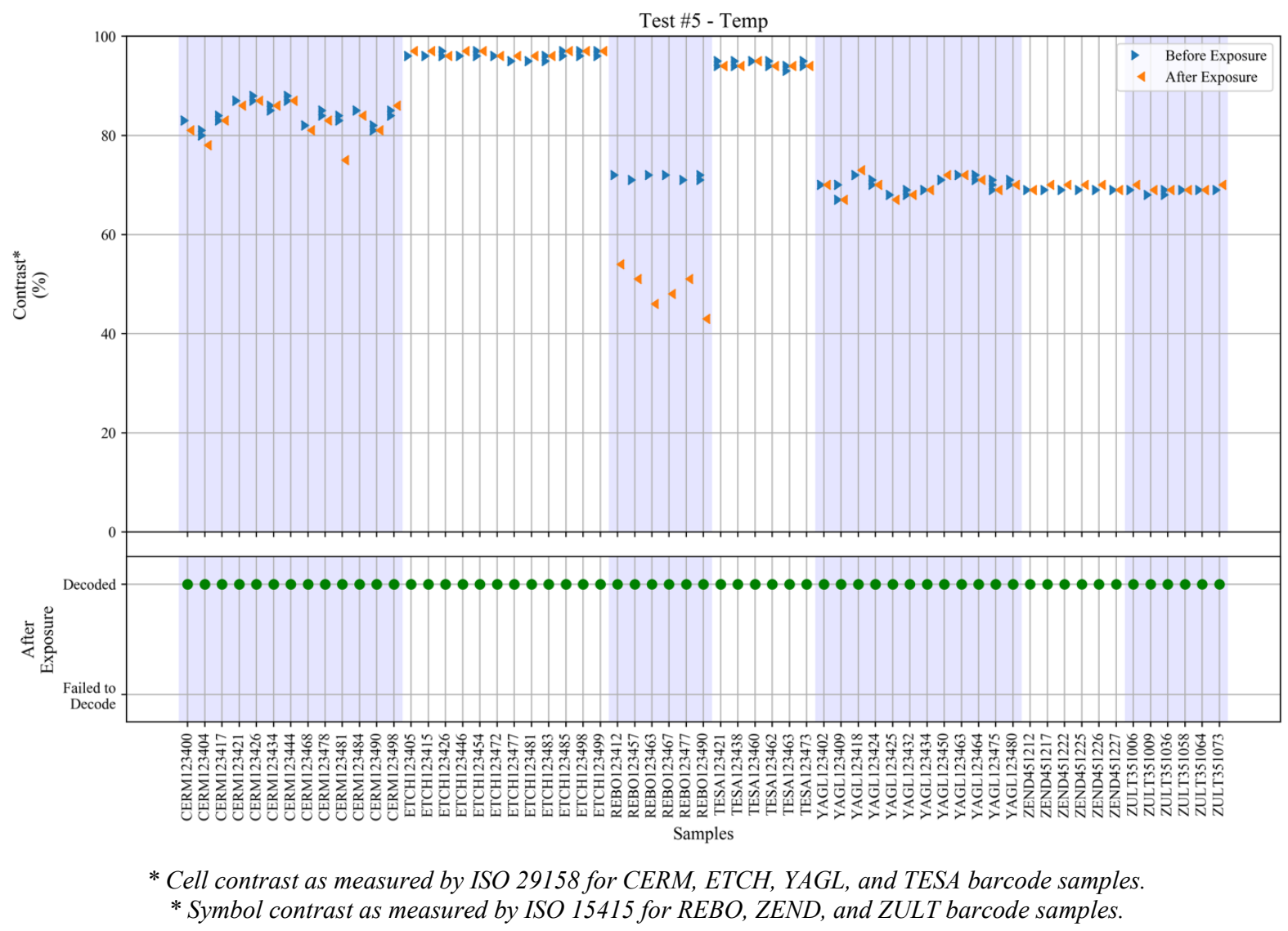

Figure 36. Cell or symbol contrast for barcode samples before and after temperature testing.

As described in the qualitative section above, the REBO sample set discolored as a result of this cyclic temperature exposure. It is not clear why this discoloration occurred. Because the sample background darkened from white to reddish-brown after exposure, the levels of contrast between dark and light elements of the barcode decreased, correlating to a significant decrease in measured symbol contrast. 


\subsection{CYCLIC CORROSION TESTING (TEST 6)}

\subsubsection{Qualitative}

Figure 37 shows the 63 barcode samples before (top) and after (bottom) exposure to cyclic corrosion testing. As expected, the A516 coupons experienced significant rust as a result of the salt fog. A rusty residue is present on all coupons in a uniform pattern, regardless of sample type or how it was affixed. We believe rust was transported through the moist fog and deposited uniformly on the flat surface of the specimens as shown in Figure 38. As described in the next section, one or more barcodes failed to decode after exposure. The authors suspected the barcodes were failing to decode because of an excessive amount of rusty residue on the samples. As a result each barcode sample was hand cleaned and rescanned. Figure 39 shows the barcode samples after hand cleaning. 

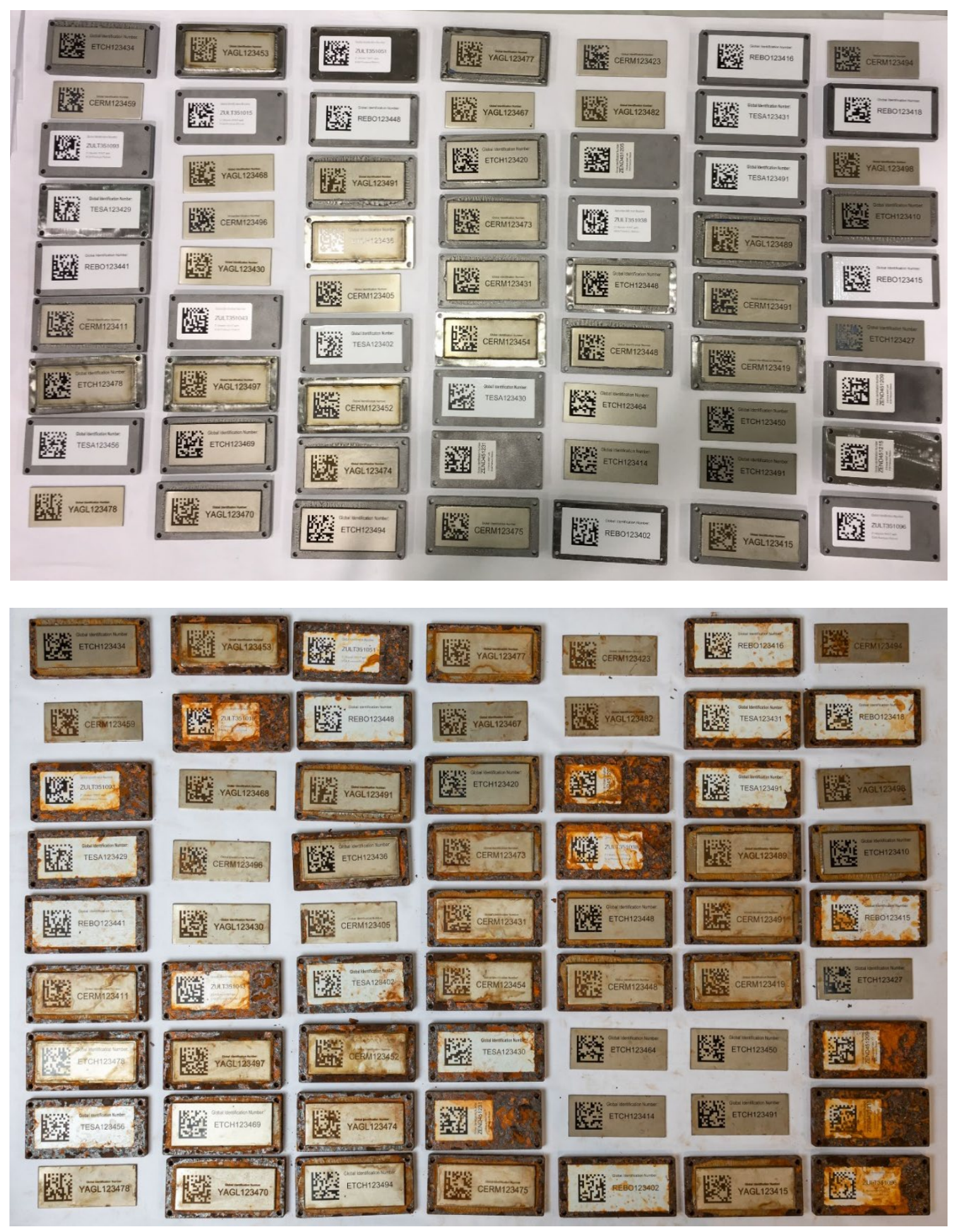

Figure 37. Barcode samples before (top) and after (bottom) cyclic corrosion testing. 


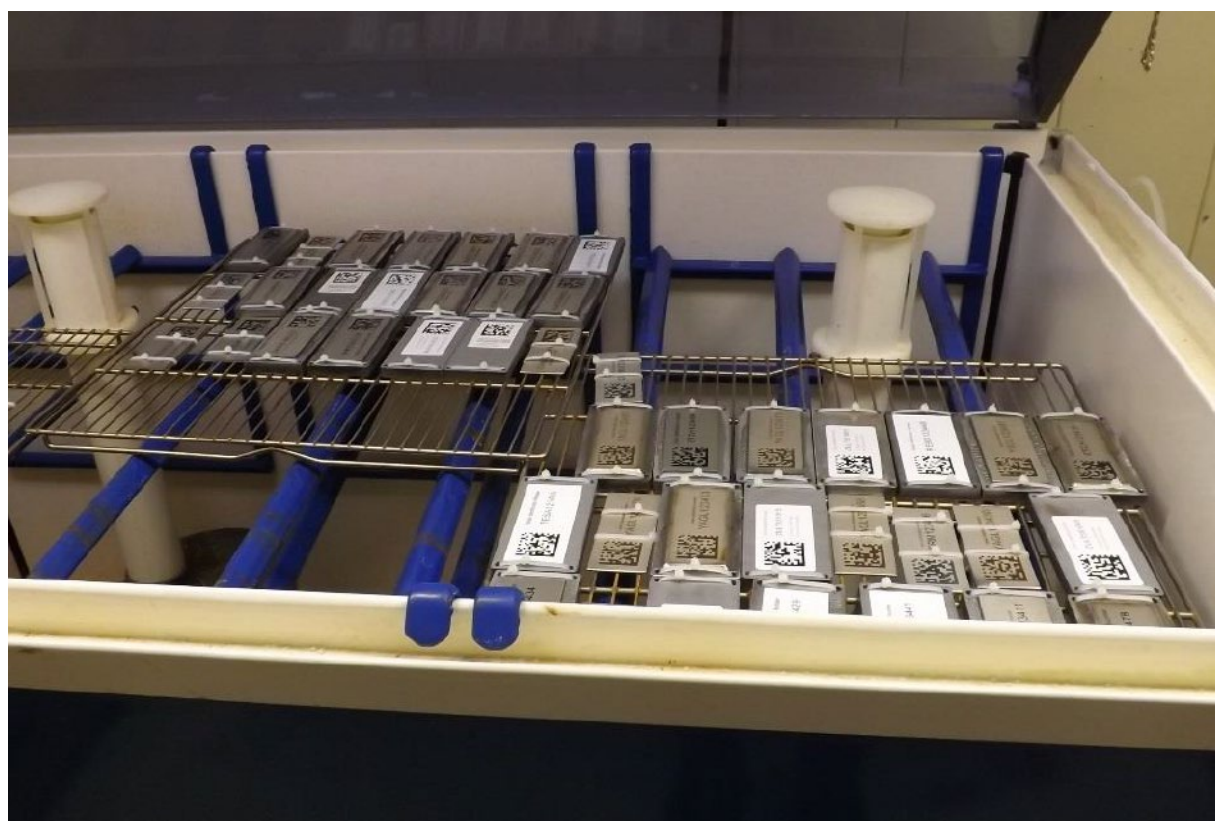

Figure 38. Barcode samples installed in GTL's salt fog chamber before ambient stage with stress exposure.

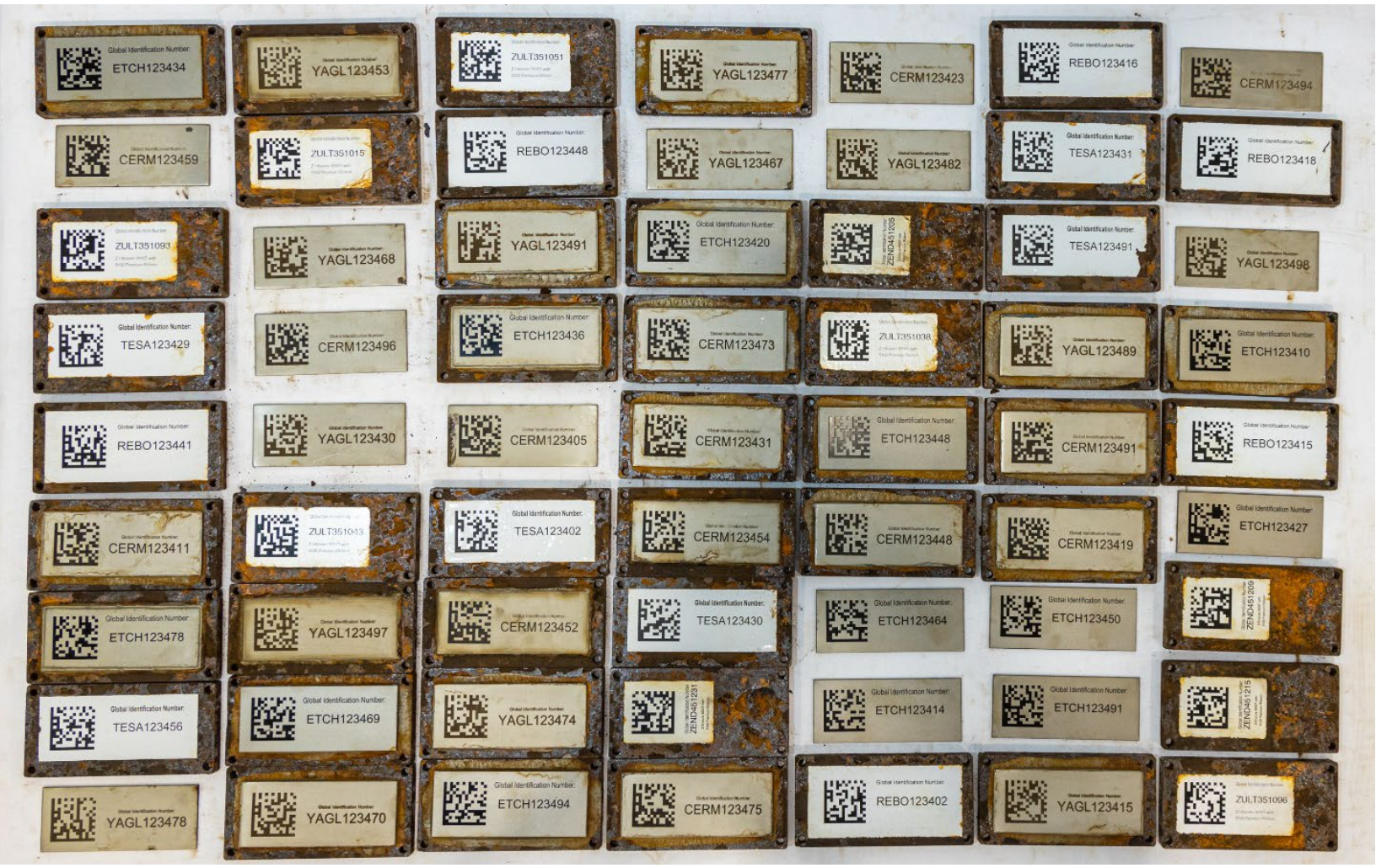

Figure 39. Barcode samples subjected to cyclic corrosion testing after hand cleaning. 


\subsubsection{Quantitative}

After exposure, 53 samples successfully decoded, but 10 samples failed to decode.

- 6 stainless-steel samples failed to decode.
○ 5 CERM
- 1 YAGL

- 4 label-type samples failed to decode.
○ 2 ZEND
○ 2 ZULT

Because one or more barcodes failed to decode after exposure, the rusty residue on the samples was cleaned as described in Section 5.4.1, and each sample was scanned again. After cleaning, the barcode verifier successfully decoded all 63 samples. Figure 39 shows each sample after cleaning. Figure 40 shows the cell or symbol contrast metric before exposure, after exposure, and after cleaning for samples subjected to cyclic corrosion testing.

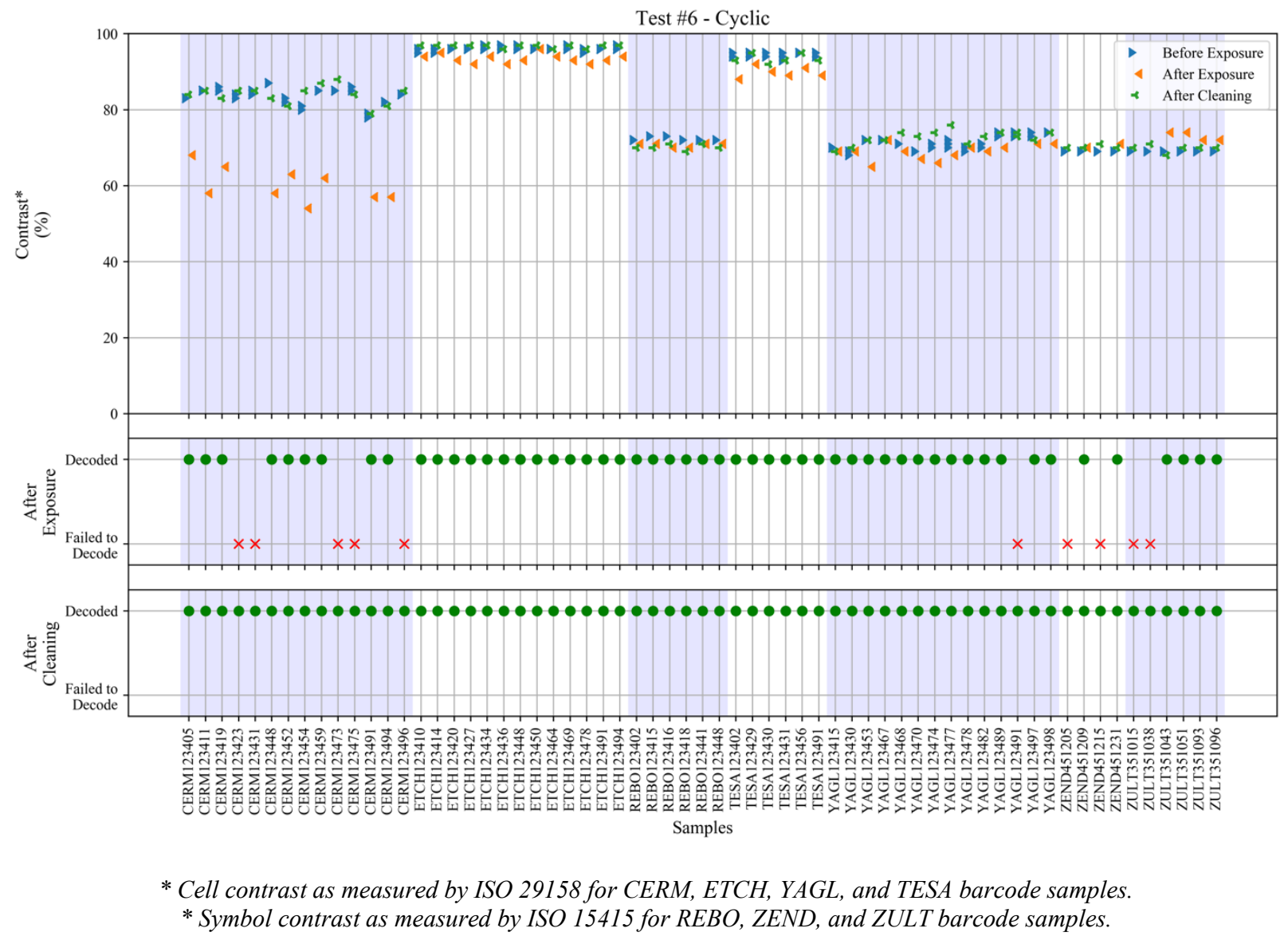

Figure 40. Before exposure, after exposure, and after cleaning cell or symbol contrast for barcode samples subjected to cyclic corrosion testing.

The CERM, ETCH, YAGL, and TESA sample sets all had a noticeable decrease in cell contrast after exposure and before cleaning, while the symbol contrast of REBO, ZEND, and ZULT sample sets did not 
significantly decrease. However, after the samples were cleaned, the barcode verifier measure little to no cell or symbol contrast degradation for each sample.

\subsection{CORROSION TESTING (TEST 7)}

\subsubsection{Qualitative}

Figure 41 shows the 57 barcode samples before (top) and after (bottom) exposure to corrosion (salt fog) testing. As shown, the A516 coupons rusted considerably. Rusty residue was present on all samples, regardless of sample type or attachment method.

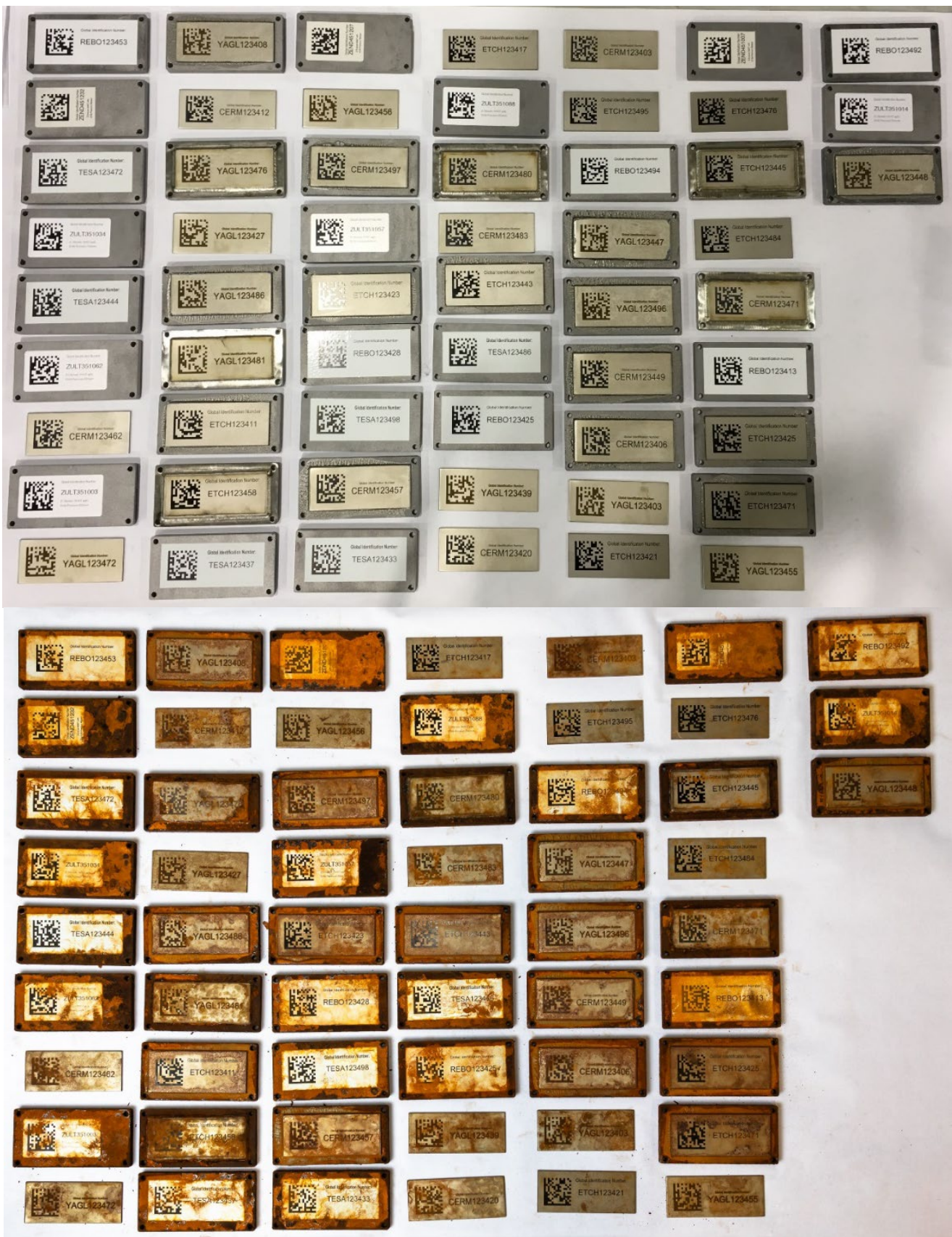

Figure 41. Barcode samples before (top) and after (bottom) corrosion testing. 
Because of the heavy amount of rust present on the samples, each barcode sample was hand cleaned as described in Section 5.4.1. Figure 42 shows each sample after cleaning; all samples were then scanned again with the barcode verifier.

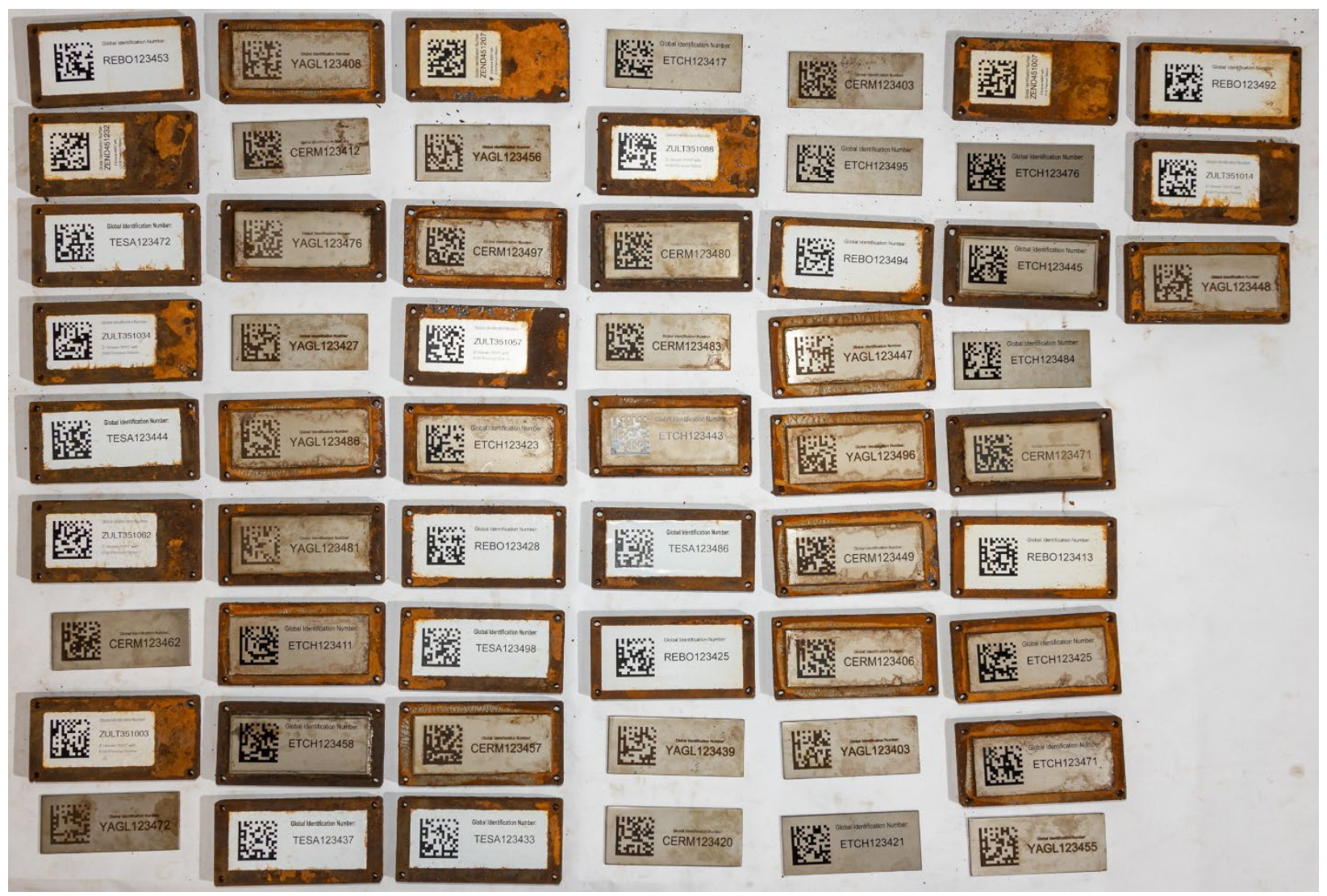

Figure 42. Barcode samples subjected to corrosion testing after cleaning.

\subsubsection{Quantitative}

After exposure, 30 of the 57 samples failed to decode with the barcode verifier. 22 stainless-steel samples failed to decode.

$\begin{array}{ll}\circ & 10 \mathrm{CERM} \\ \circ & 5 \mathrm{ETCH} \\ \circ & 7 \mathrm{YAGL}\end{array}$

- 8 label-type samples failed to decode.
- 2 REBO
- 2 TESA
- 2 ZEND
- 2 ZULT

After exposure, but before cleaning, the samples that were decoded showed a decrease in cell or symbol contrast. The 14 CERM, ETCH, and YAGL samples that the barcode verifier decoded demonstrated the largest decrease in cell contrast after testing before cleaning when compared to the other sample types. We believe the ball-blasted finish of stainless steel left a porous surface for the rust residue to adhere to and impacted contrast for these sample types. 
After cleaning, two YAGL samples did not decode: YAGL123476 and YAGL123486. Figure 43 shows the cell or symbol contrast metric before exposure, after exposure, and after cleaning for samples subjected to corrosion testing.

After the samples were hand cleaned, 55 of the 57 samples showed a significantly smaller decrease in cell or symbol contrast compared to their before-exposure values. It is not immediately clear why YAGL123476 and YAGL123486 did not decode after hand cleaning.

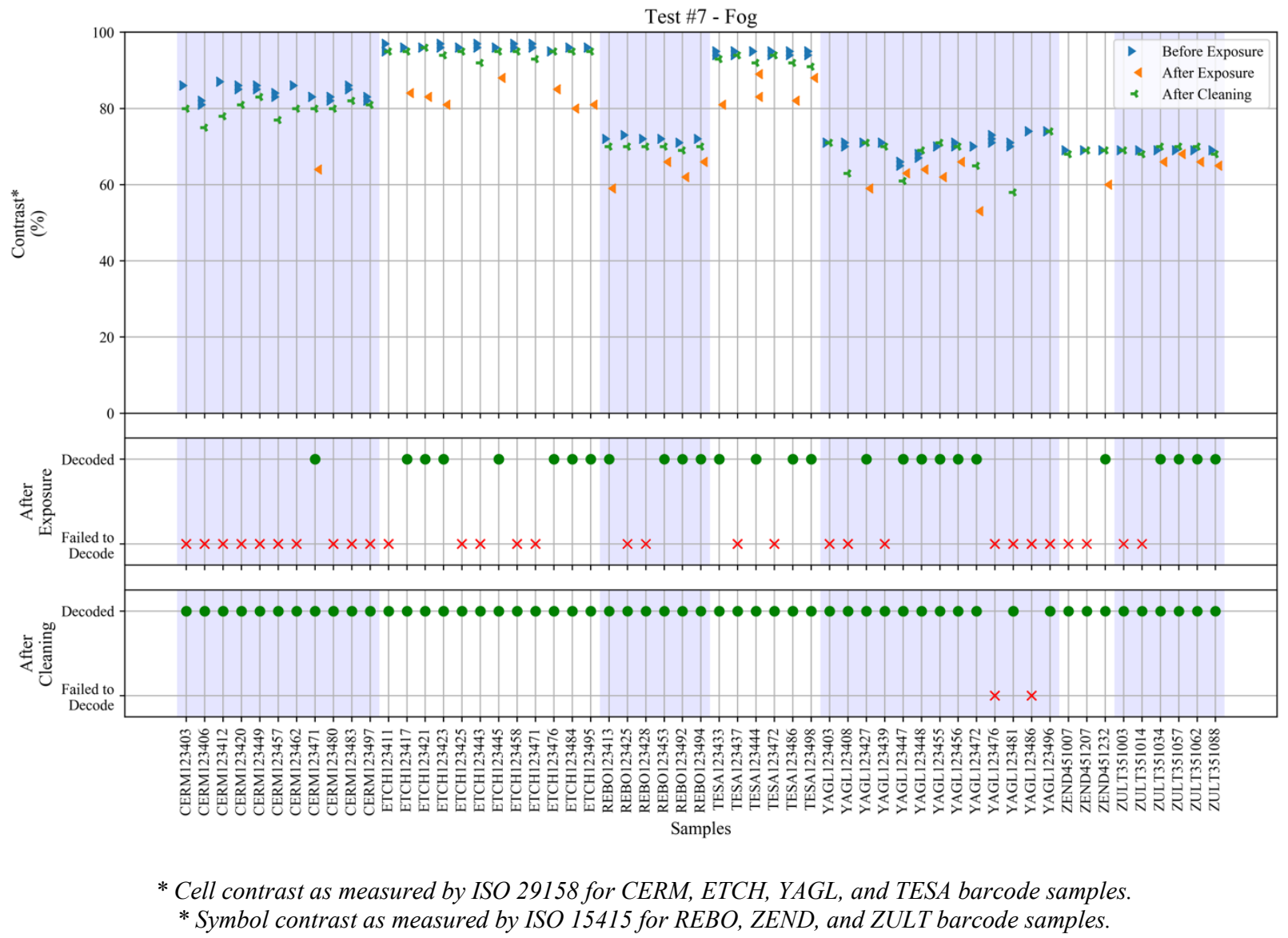

Figure 43. Before exposure, after exposure, and after cleaning cell or symbol contrast for barcode samples subjected to corrosion testing. 


\subsection{BLOWING SAND/DUST TESTING (TEST 8)}

\subsubsection{Qualitative}

Figure 44 shows the 57 barcode samples before (top) and after (bottom) exposure to blowing sand and dust testing. The A516 coupons (mainly the polished coupons) show mild signs of oxidation. Any oxidation patterns did not have a significant effect on the barcode decoding.
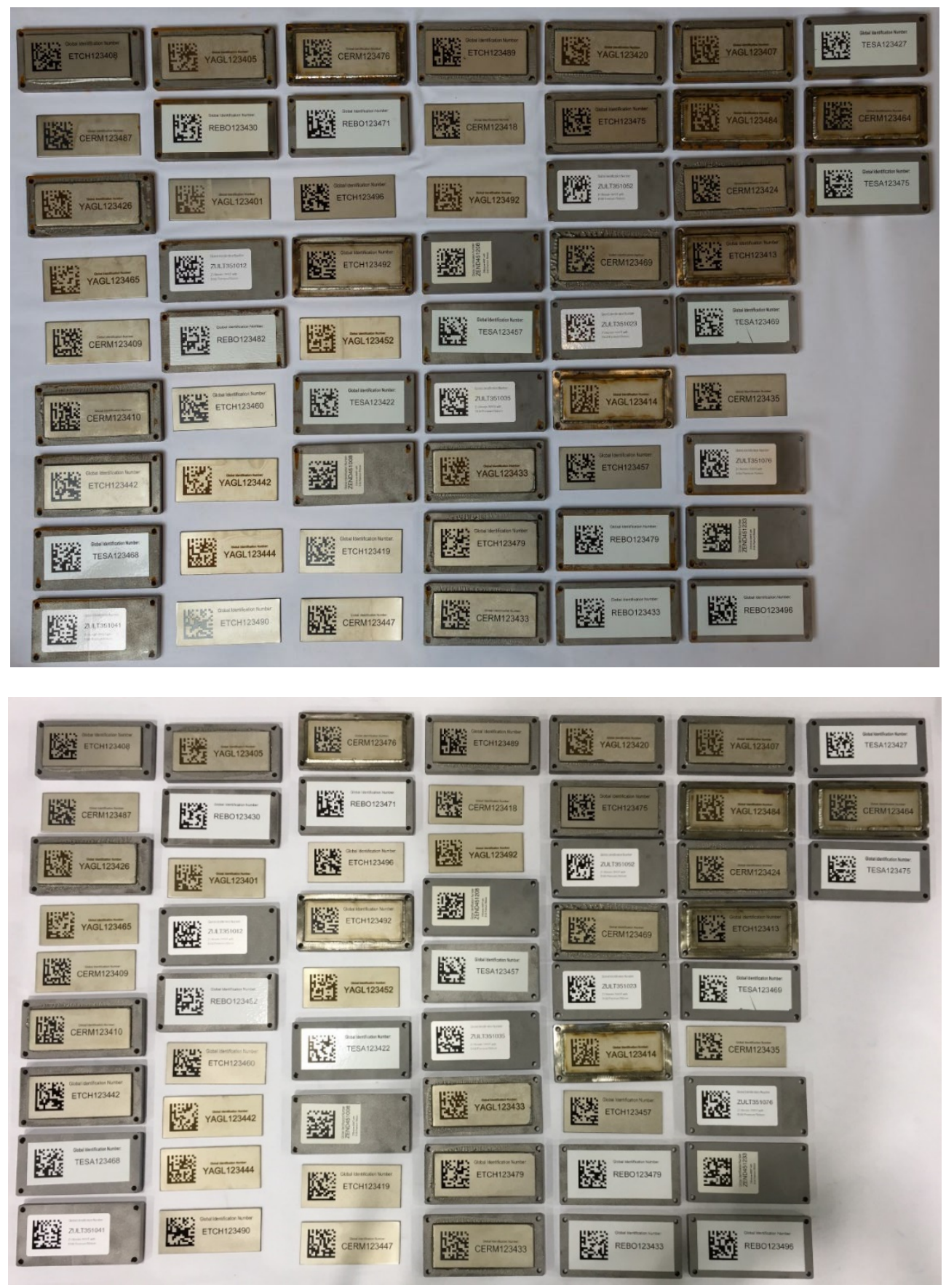

Figure 44. Barcode samples before (top) and after (bottom) blowing sand/dust testing. 


\subsubsection{Quantitative}

After exposure, all 57 samples were successfully decoded by the barcode verifier. Figure 45 shows the cell or symbol contrast metric before and after exposure for samples subjected to blowing sand and dust testing. A significant decrease in cell or symbol contrast did not occur as a result of testing. The decrease in cell or symbol contrast was minimal for label-type samples (REBO, TESA, ZEND, ZULT), and almost no decrease in cell contrast was measured for stainless-steel samples (CERM, ETCH, YAGL). One noted outlier was the YAGL123401 sample, which demonstrated a sizable decrease in cell contrast after exposure. It is not clear why this decrease occurred.

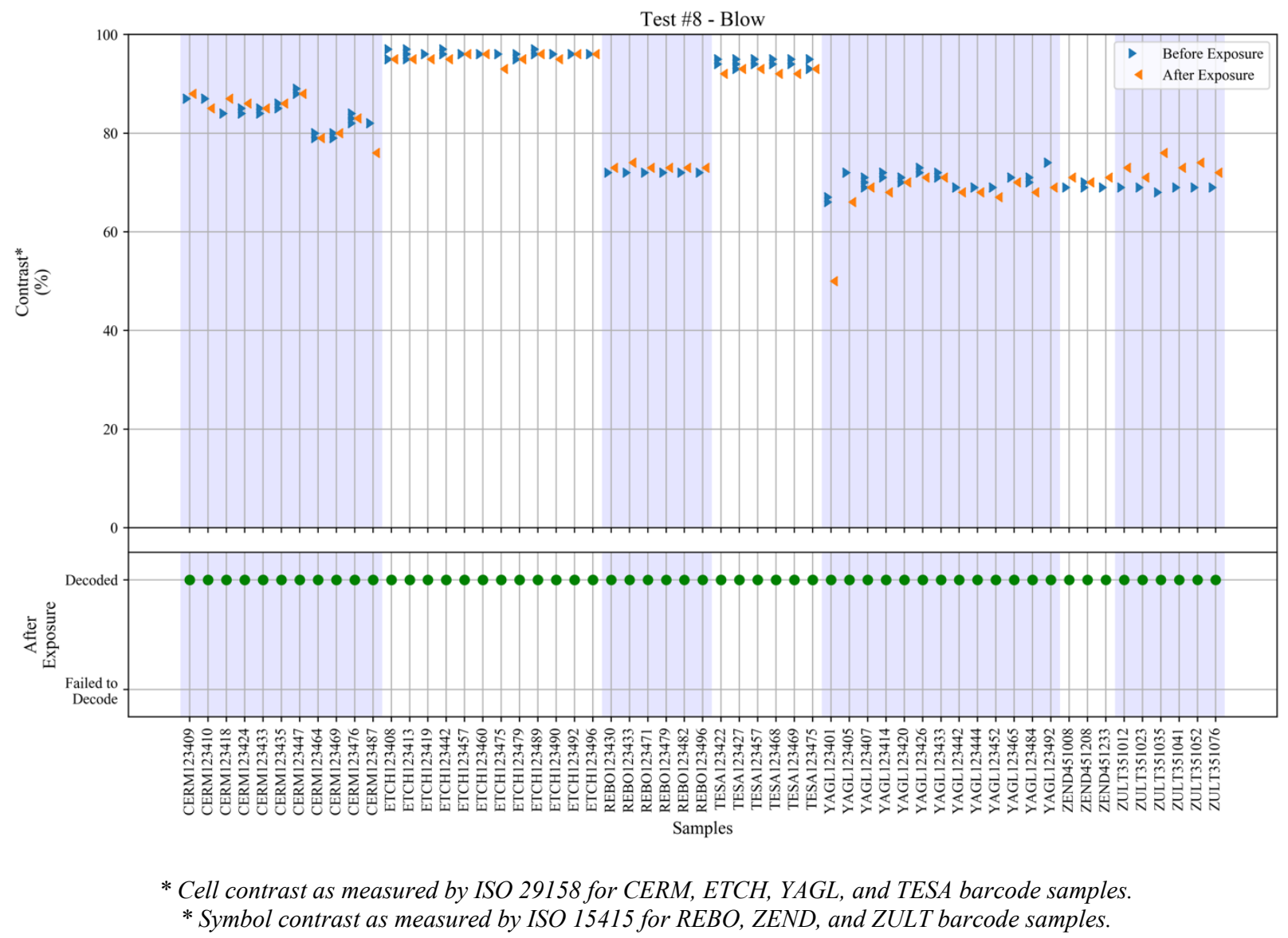

Figure 45. Cell or symbol contrast for barcode samples before and after blowing sand/dust testing. 


\subsection{IMPACT TESTING (TEST 9)}

\subsubsection{Qualitative}

Figure 46 shows the 55 barcode samples before (top) and after (bottom) after exposure to impact testing. Similar to the samples in Test 8, the A516 coupons (mainly the polished coupons) showed mild signs of oxidation. Any oxidation patterns did not have a significant effect on the barcode decoding.
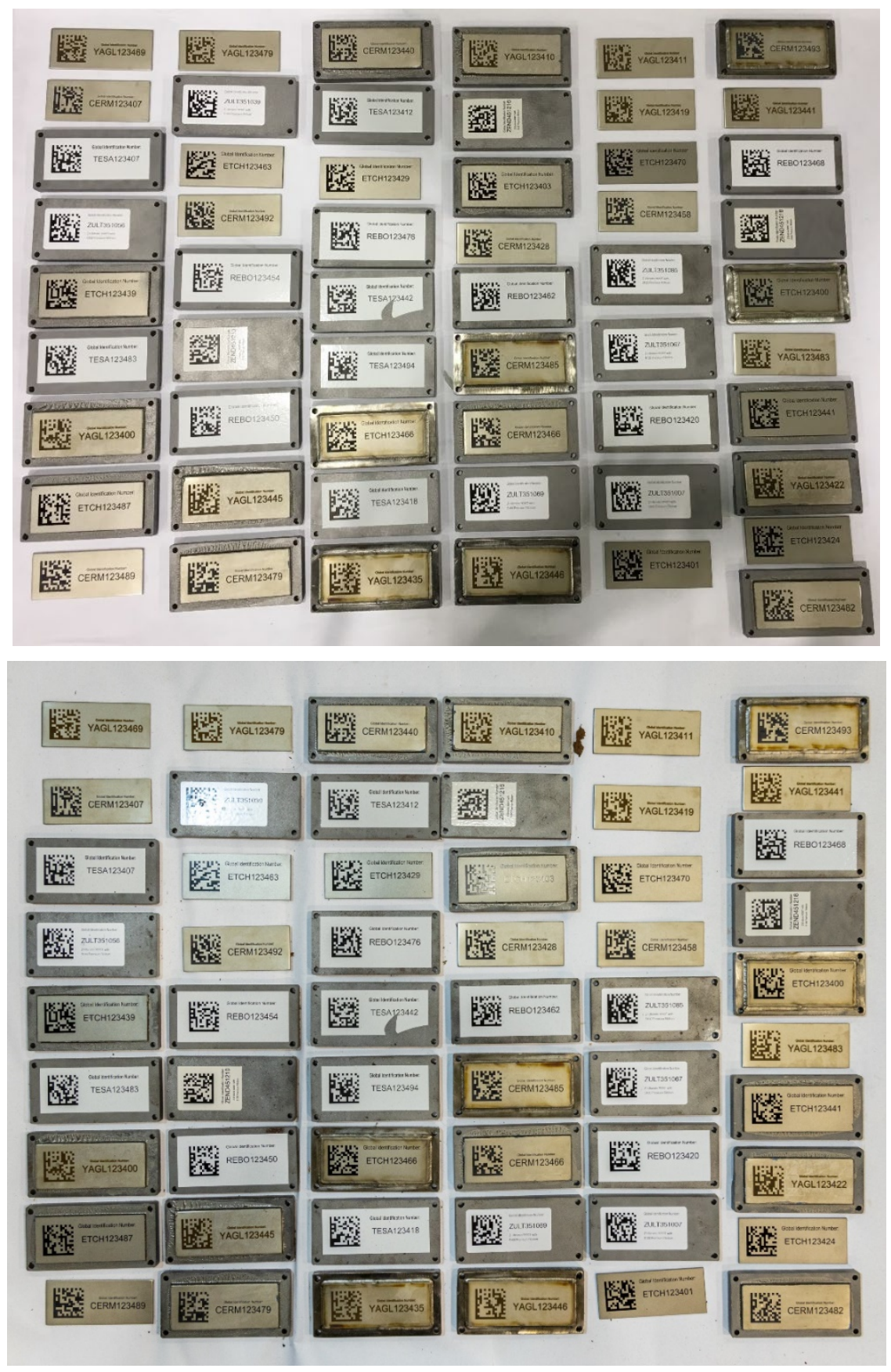

Figure 46. Barcode samples before (top) and after (bottom) impact testing. 


\subsubsection{Quantitative}

After exposure, all 55 samples were successfully decoded with the barcode verifier. Figure 47 shows the cell or symbol contrast metric before and after exposure for samples subjected to impact testing. A significant decrease in cell or symbol contrast did not occur as a result of testing. There was a slightly larger decrease in cell contrast for the YAGL samples compared to all others; however, the decrease was not significant.

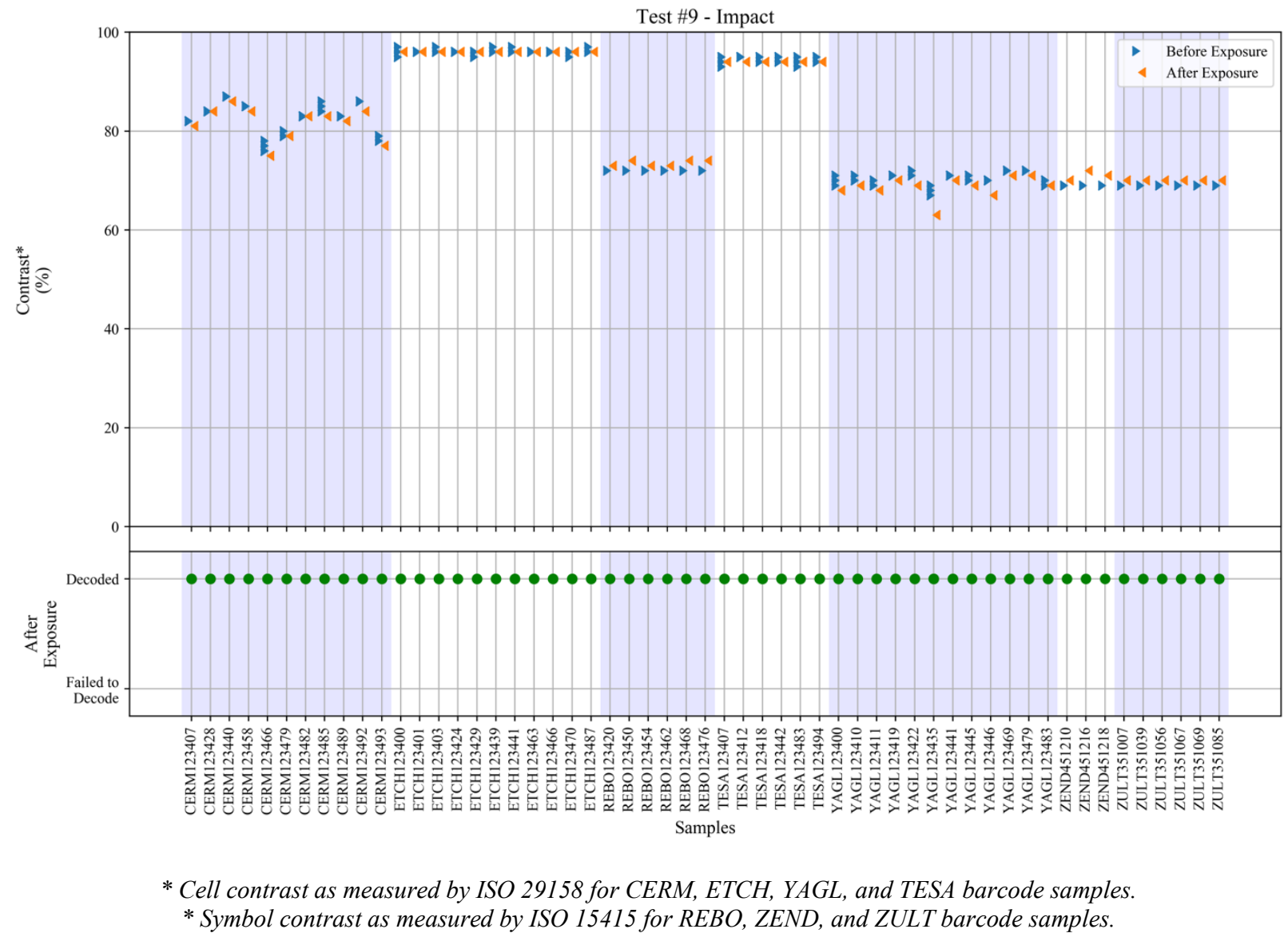

Figure 47. Cell or symbol contrast for barcode samples before and after impact testing. 


\subsection{HIGH PRESSURE AND TEMPERATURE WATER JET (TEST 10)}

\subsubsection{Qualitative}

Figure 48 shows the 57 barcode samples before (top) and after (bottom) exposure to high pressure and temperature water jet testing. The A516 coupons show moderate signs of oxidation. These oxidation patterns are predominantly surface rust patterns, likely attributed to the rust from the introduction of moisture during testing. This did not have significant effect on the sample scans.

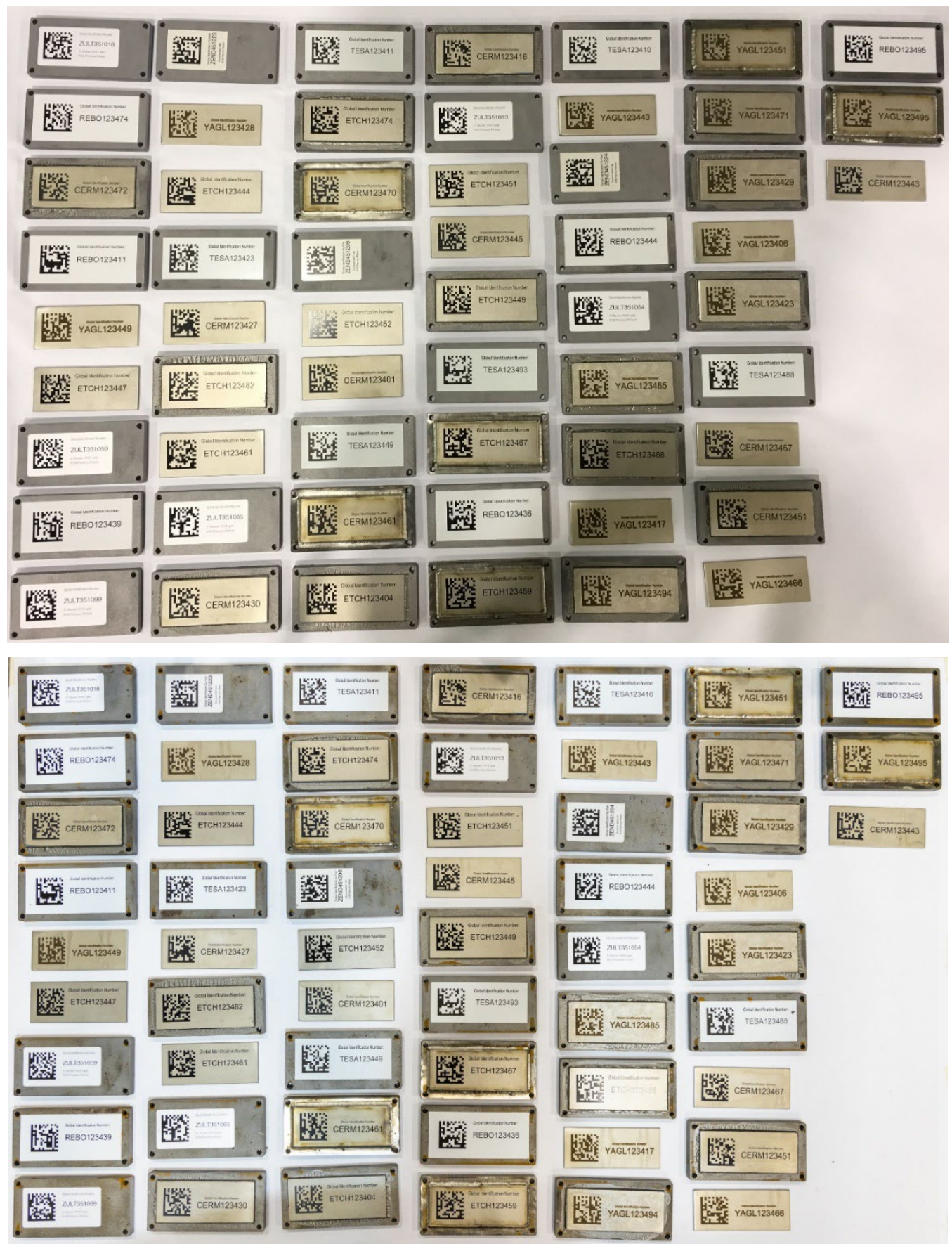

Figure 48. Barcode samples before (top) and after (bottom) high pressure and temperature water jet testing. 


\subsubsection{Quantitative}

After exposure, 54 of the 55 samples were successfully decoded with the barcode verifier. Based on visual inspection, we determined that ZULT351099 had ripped at some point between the pre-exposure scan and the postexposure scan. Figure 49 shows ZULT351099 with the rip circled in red.

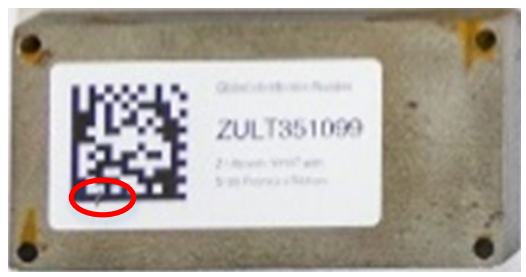

Figure 49. ZULT351099 shown with ripped barcode circled in red.

Figure 50 shows the cell or symbol contrast metric before and after exposure for samples subjected to high-pressure and temperature water jet testing. The cell contrast did not significantly change for the CERM, ETCH, TESA, or ZEND samples. The contrast decreased slightly for the YAGL samples. The team failed to scan the REBO and ZULT samples before they were shipped, so the before-exposure data is not shown in Figure 50.

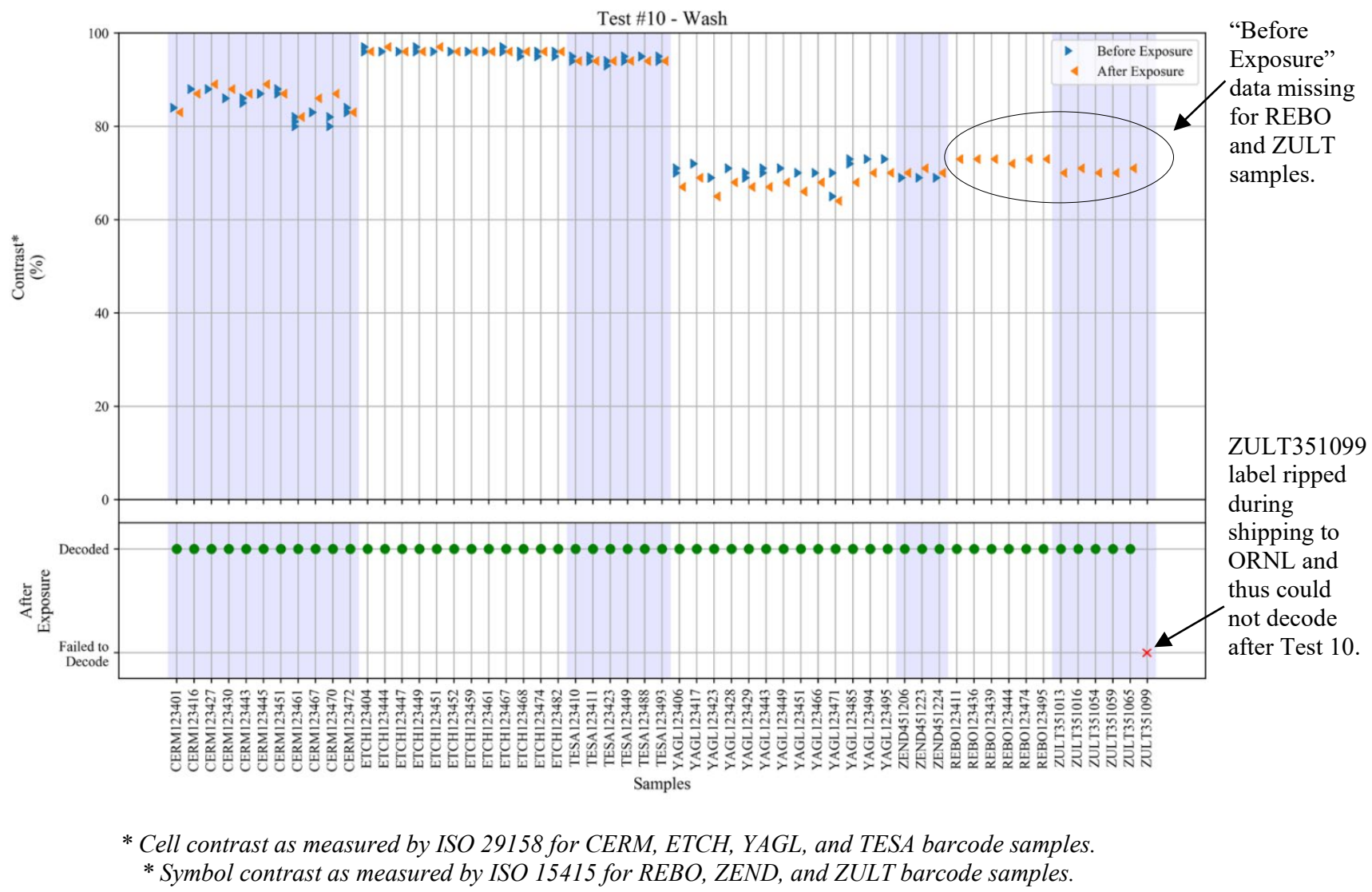

Figure 50. Cell or symbol contrast for barcode samples before and after high pressure and temperature water jet testing. 


\subsection{SUMMARY OF RESULTS BY TEST}

After cleaning samples from some of the tests, the temperature testing had the largest effect on the contrast of the samples on average, and the cyclic tests had the smallest effect (Table 4).

Table 4. Summary of cell or symbol contrast values for each test before exposure, after exposure, and after cleaning (sorted by change in contrast).

\begin{tabular}{lcccccc}
\hline \multicolumn{1}{c}{ Test } & $\begin{array}{c}\text { Before } \\
\text { Exposure }\end{array}$ & $\begin{array}{c}\text { After } \\
\text { Exposure }\end{array}$ & $\begin{array}{c}\text { After } \\
\text { Cleaning }\end{array}$ & $\begin{array}{c}\text { Difference } \\
\text { After } \\
\text { Exposure }\end{array}$ & $\begin{array}{c}\text { Difference } \\
\text { After } \\
\text { Cleaning }\end{array}$ & $\begin{array}{c}\text { Final } \\
\text { Difference }\end{array}$ \\
\hline 5 - Temp & 79.58 & 75.91 & - & $(3.67)$ & - & $(3.67)$ \\
7 - Fog & 79.84 & 68.87 & 77.46 & $(10.97)$ & $(2.38)$ & $(2.38)$ \\
2 - QUV (UVA) & 80.03 & 77.73 & - & $(2.30)$ & - & $(2.30)$ \\
10 - Wash & 81.70 & 79.63 & - & $(2.07)$ & - & $(2.07)$ \\
4 - QUV \& Q-FOG & 80.18 & 76.17 & 78.57 & $(4.01)$ & $(1.61)$ & $(1.61)$ \\
1 - Q-Sun UV & 79.83 & 78.65 & - & $(1.18)$ & - & $(1.18)$ \\
3 - QUV (UVB) & 79.77 & 79.14 & - & $(0.63)$ & - & $(0.63)$ \\
8 - Blow & 79.75 & 79.42 & - & $(0.33)$ & - & $(0.33)$ \\
9 - Impact & 79.49 & 79.26 & - & $(0.24)$ & - & $(0.24)$ \\
6 - Cyclic & 79.82 & 75.23 & 79.59 & $(4.59)$ & $(0.23)$ & $(0.23)$ \\
\hline
\end{tabular}




\section{RESULTS BY BARCODE SAMPLE TYPE}

This section describes the overall performance of each of the 7 types of samples to the 10 environmental tests that were performed.

\subsection{CERM STAINLESS-STEEL SAMPLES}

Figure 51 shows the cell contrast metric for the 87 CERM samples evaluated before and after each environmental test. As shown in the previous sections for tests with corrosion, Tests 4, 6, and 7 also include data points for cell contrast before and after sample cleaning. The samples subjected to the remaining tests (Tests $1,2,3,5,8,9$, and 10) were not hand cleaned and rescanned.

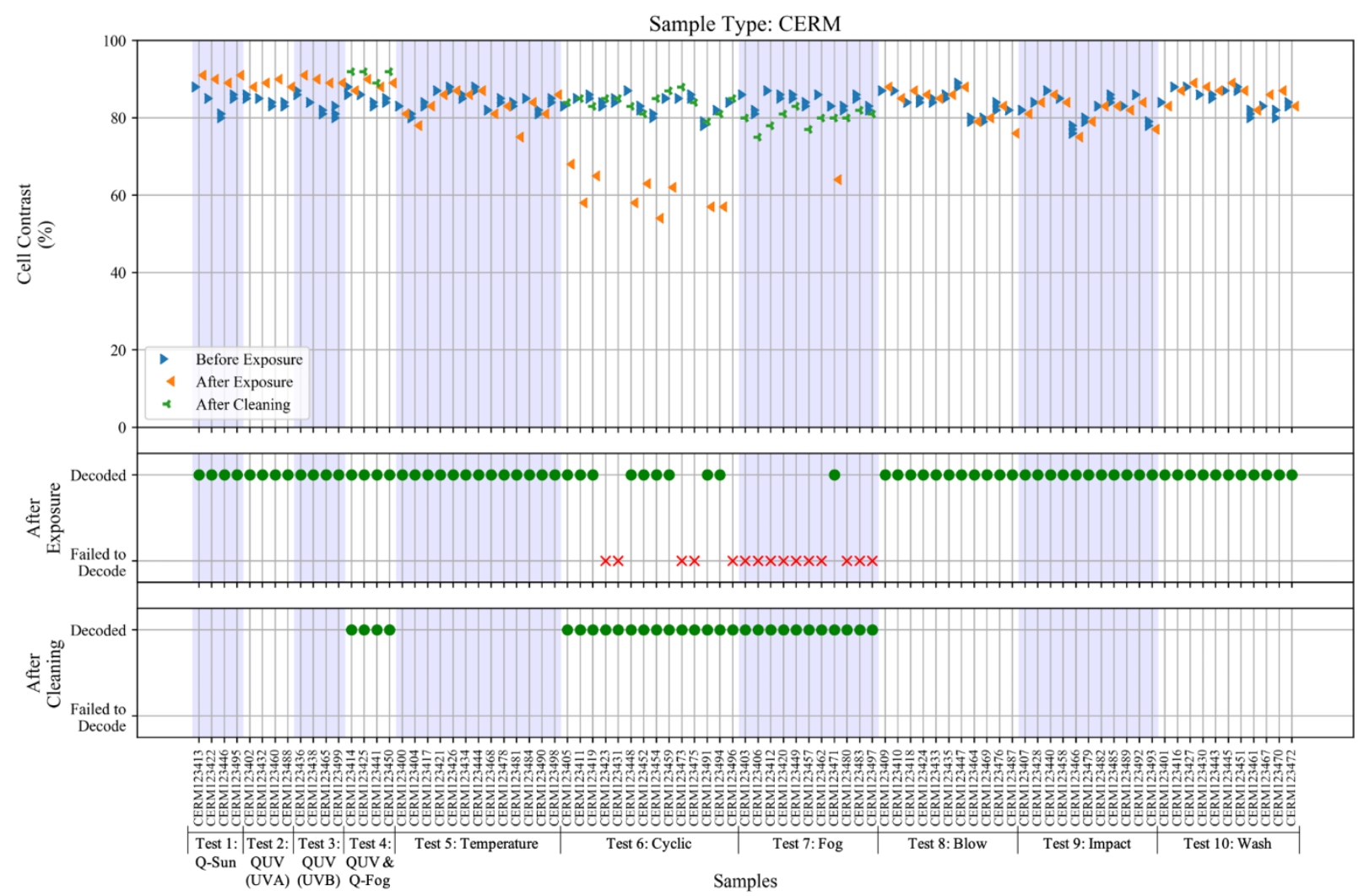

Figure 51. Cell contrast for CerMark-coated laser-marked stainless-steel (CERM) barcode samples before and after all environmental tests.

The CERM samples were significantly affected by the corrosion-based environmental tests. The CERM samples performed the worst when exposed to salt fog (Test 7), with only 1 of the 11 samples decoding before hand cleaning. For the samples that decoded across all tests, the after-exposure cell contrast decrease was largest after cyclic testing (Test 6), which was likely caused by the presence of rust on the barcodes. After the samples subjected to Tests 4, 6, and 7 were hand cleaned, all barcodes in Tests 6 and 7 decoded, and the samples subjected to Test 6 showed little to no change in cell contrast. Test 7 samples showed the highest decrease in cell contrast, but the after-exposure values are still within $10 \%$ of the before-exposure values for all samples. Note, all 87 CERM sample identification numbers could be manually read by an ORNL researcher both before and after hand cleaning, regardless of the sample's ability to decode. 


\subsection{ETCH STAINLESS-STEEL SAMPLES}

Figure 52 shows the cell contrast metric for the $87 \mathrm{ETCH}$ samples evaluated before and after each environmental test. As shown in the previous sections for tests with corrosion, Tests 4, 6, and 7 also include data points for cell contrast before and after sample cleaning. The samples subjected to the remaining tests (Tests $1,2,3,5,8,9$, and 10) were not hand cleaned and rescanned.

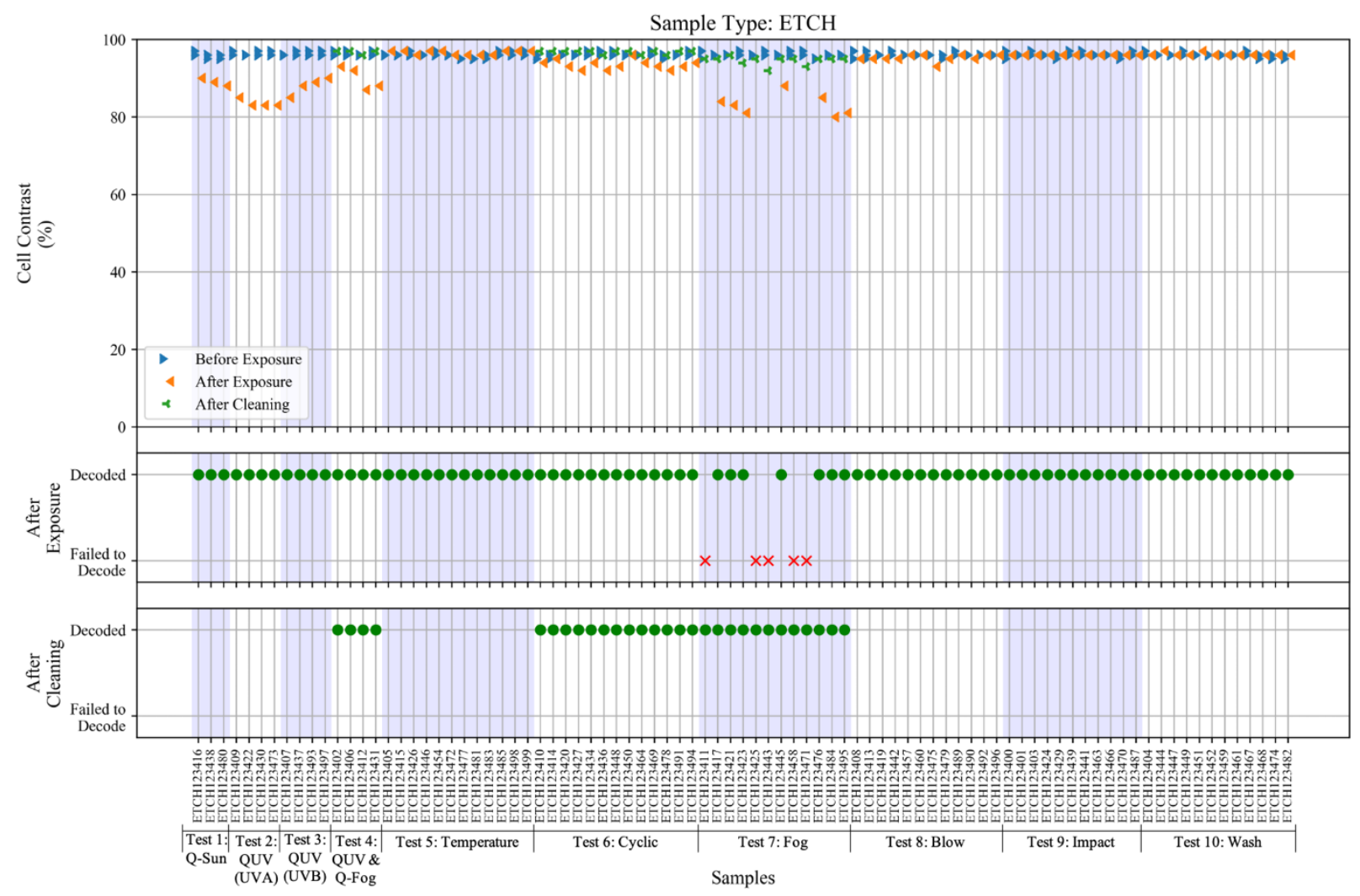

Figure 52. Cell contrast for chemically etched stainless-steel (ETCH) barcode samples before and after all environmental tests.

When compared across all test types, the ETCH samples show a susceptibility to UV degradation, with the largest decreases in cell contrast seen after UVA exposure during Test 2. Corrosion-based tests also caused a significant decrease in cell contrast, which was visible for the samples exposed to Tests 6 and 7 . Five of the twelve samples exposed to Test 7 did not decode after environmental exposure. After hand cleaning, all 12 samples exposed to Test 7 successfully decoded, and a significant change in the cell contrast was not attributable to the corrosion of the barcode. Thus, it can be assumed that the rust dust present on the coupons before hand cleaning was the source of cell contrast decrease. Note, all $87 \mathrm{ETCH}$ sample identification numbers could be manually read by an ORNL researcher both before and after hand cleaning, regardless of the sample's ability to decode.

\subsection{YAGL STAINLESS-STEEL SAMPLES}

Figure 53 shows the cell contrast metric for the 92 YAGL samples evaluated before and after each environmental test. As shown in the previous sections for tests with corrosion, Tests 4, 6, and 7 also include data points for cell contrast before and after sample cleaning. The samples subjected to the remaining tests (Tests 1, 2, 3, 5, 8, 9, and 10) were not hand cleaned and rescanned. 


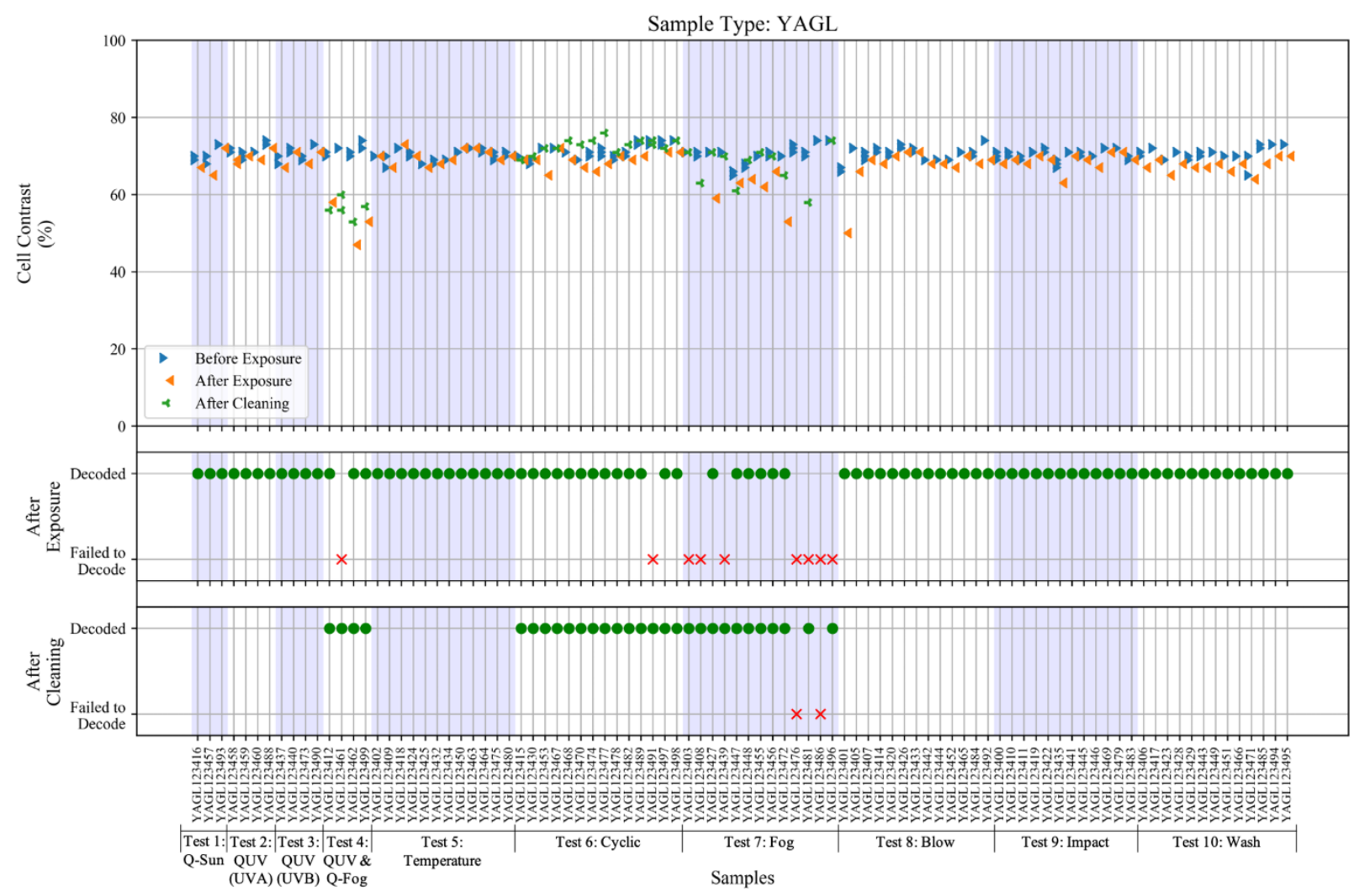

Figure 53. Cell contrast for laser-marked stainless-steel (YAGL) barcode samples before and after all environmental tests.

Overall, the YAGL samples have a lower before-exposure cell contrast than other stainless-steel samples (CERM and ETCH). The YAGL samples appear very succeptable to the combination of UV and salt fog exposure as demonstrated in Test 4. YAGL samples subjected to Test 7 were the only samples that could not be decoded after being hand cleaned (e.g., YAGL123476 and YAGL123486). Note, all 92 YAGL sample identification numbers could be manually read by an ORNL researcher both before and after hand cleaning, regardless of the sample's ability to decode.

\subsection{REBO LABEL-TYPE SAMPLES}

Figure 54 shows the symbol contrast metric for the 48 REBO samples evaluated before and after each environmental test. As shown in the previous sections for tests with corrosion, Tests 4, 6, and 7 also include data points for symbol contrast before and after sample cleaning. The samples subjected to the remaining tests (Tests $1,2,3,5,8,9$, and 10) were not hand cleaned and rescanned. 


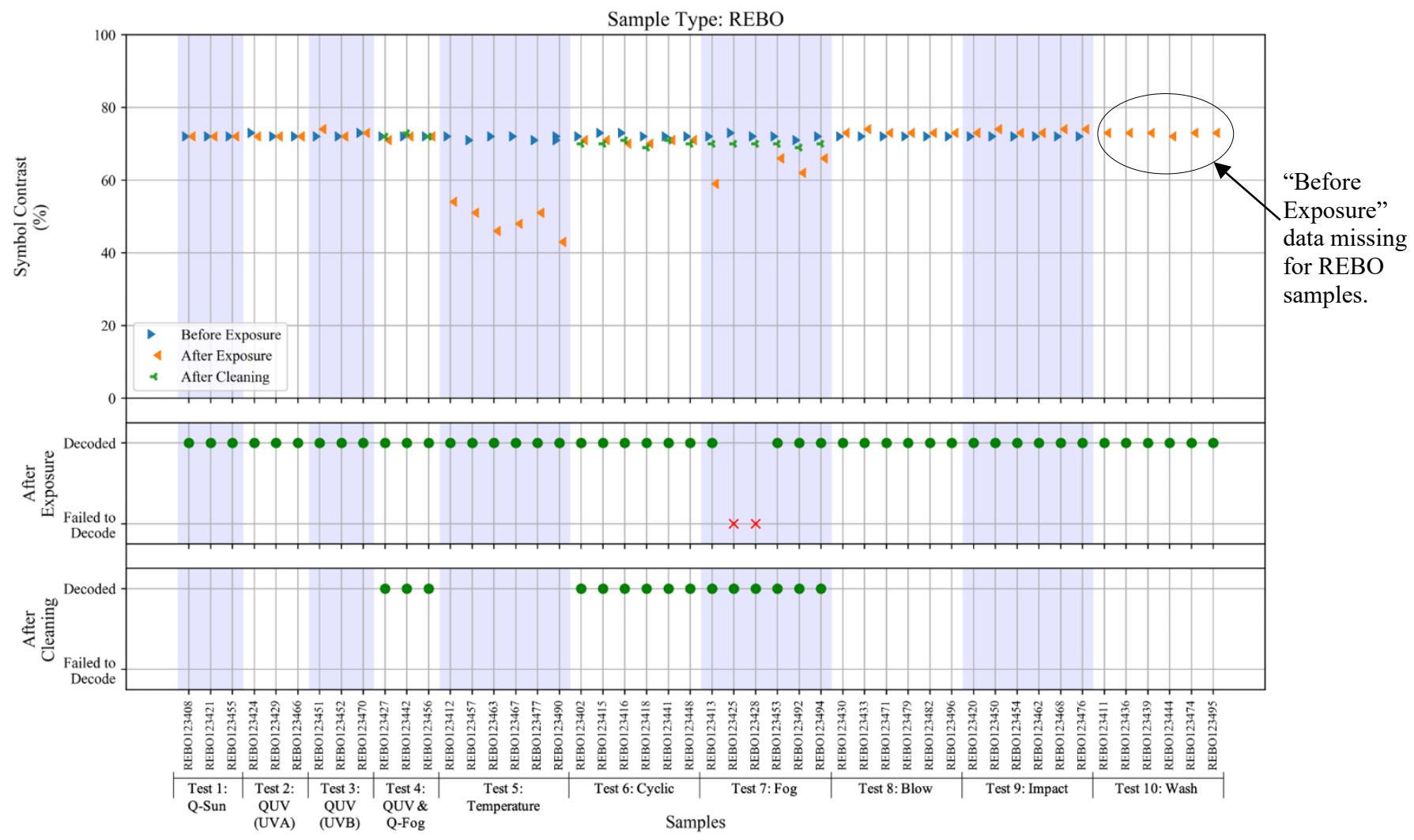

Figure 54. Symbol contrast for Rebo premium vinyl label (REBO) barcode samples before and after all environmental tests.

The REBO sample set was relatively consistent across all environmental tests, with the exception of temperature exposure. For the temperature variation tests (Test 5), the REBO samples performed the worst of any sample set. As shown in Figure 23, the samples discolored as a result of the testing. This caused a significant decrease in the symbol contrast compared to all other tests, showing a distinct weakness for this sample type. For corrosion tests, the performance of the REBO samples was comparable to other label-type barcode samples (TESA, ZEND, ZULT), showing little to no symbol contrast decrease after sample cleaning.

The team failed to locate before-exposure scans for Test 10. Since the before-exposure cell contrast values are consistent across all the other tests, the authors suggest that the before-exposure symbol contrast values for the pressure and temperature water jet test were similar to the before-exposure values of REBO samples for the other tests.

Note, all 48 REBO sample identification numbers could be manually read by an ORNL researcher both before and after hand cleaning, regardless of the sample's ability to decode.

\subsection{TESA LABEL-TYPE SAMPLES}

Figure 55 shows the cell contrast metric for the 48 TESA samples evaluated before and after each environmental test. As shown in the previous sections for tests with corrosion, Tests 4, 6, and 7 also include data points for cell contrast before and after sample cleaning. The samples subjected to the remaining tests (Tests $1,2,3,5,8,9$, and 10) were not hand cleaned and rescanned. 


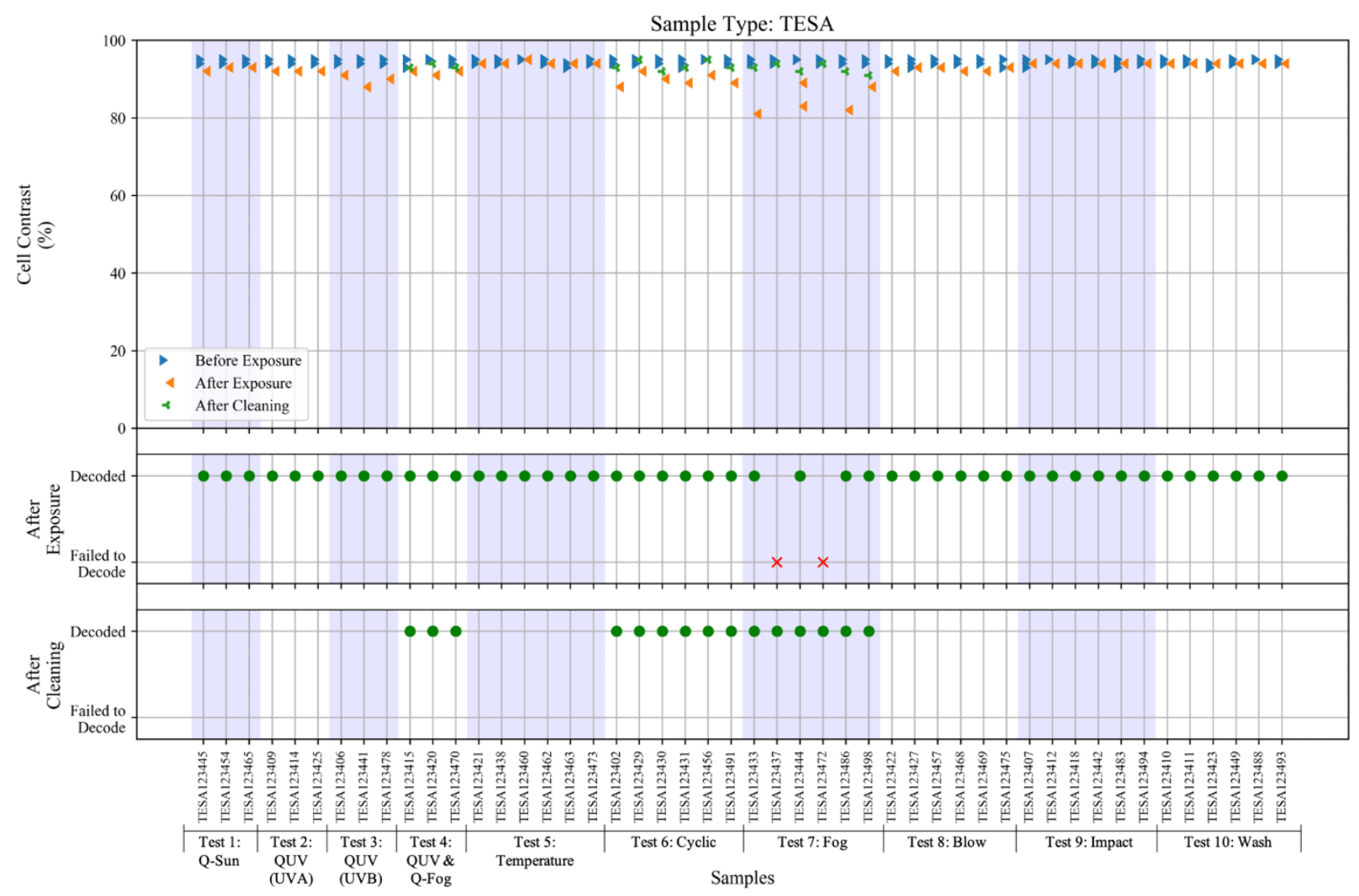

Figure 55. Cell contrast for laser-marked Tesa tape (TESA) barcode samples before and after all environmental tests.

The TESA sample set was one of the most consistent sample types. UVB exposure (Test 3) seemed to have the greatest influence on cell contrast. This was followed by salt fog exposure (Test 7), after which two of the six samples failed to decode. Note, all 48 TESA sample identification numbers could be manually read by an ORNL researcher both before and after hand cleaning, regardless of the sample's ability to decode.

\subsection{ZEND LABEL-TYPE SAMPLES}

Figure 56 shows the symbol contrast metric for the 34 ZEND samples evaluated before and after each environmental test. As shown in the previous sections for tests with corrosion, Tests 4, 6, and 7 also include data points for symbol contrast before and after sample cleaning. The samples subjected to the remaining tests (Tests $1,2,3,5,8,9$, and 10) were not hand cleaned and rescanned. 


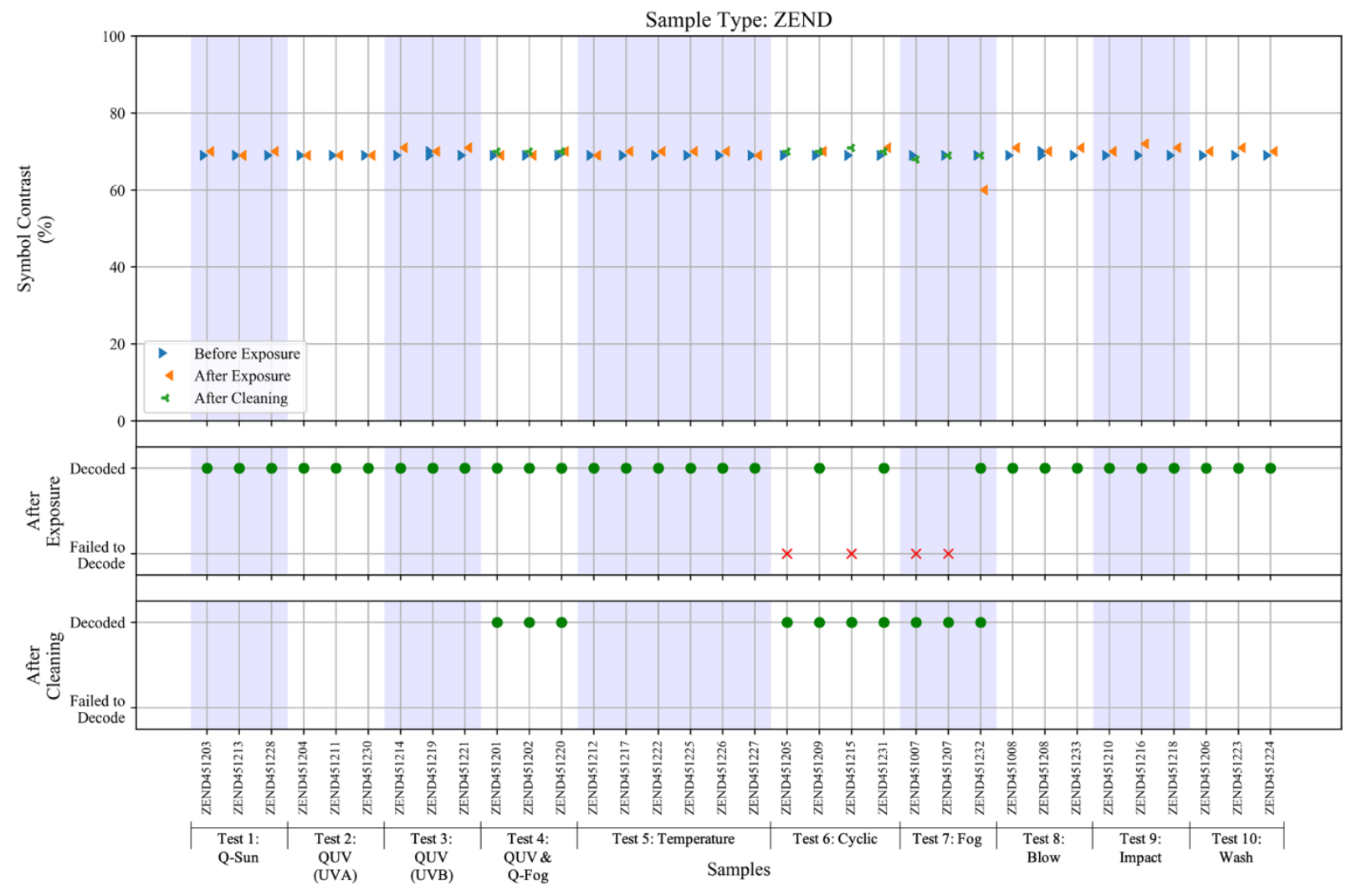

Figure 56. Symbol contrast for Zebra Z-Endure 4000T acrylic label (ZEND) barcode samples before and after all environmental tests.

Across all test types, the ZEND samples showed the most consistent symbol contrast before and after environmental tests. Although a high portion of the samples failed to decode when exposed to corrosion tests (two of the four samples in Test 6 and two of the three samples in Test 7), all seven samples in Tests 6 and 7 decoded after hand cleaning and the resulting increase in symbol contrast demonstrates that the initial decrease after-exposure comes from the coupon rust dust, not from the sample integrity. Note, all 34 ZEND sample identification numbers could be manually read by an ORNL researcher both before and after hand cleaning, regardless of the sample's ability to decode.

\subsection{ZULT LABEL-TYPE SAMPLES}

Figure 57 shows the symbol contrast metric for the 48 ZULT samples evaluated before and after each environmental test. As shown in the previous sections for tests with corrosion, Tests 4, 6, and 7 also include data points for symbol contrast before and after sample cleaning. The samples subjected to the remaining tests (Tests $1,2,3,5,8,9$, and 10) were not hand cleaned and rescanned. 


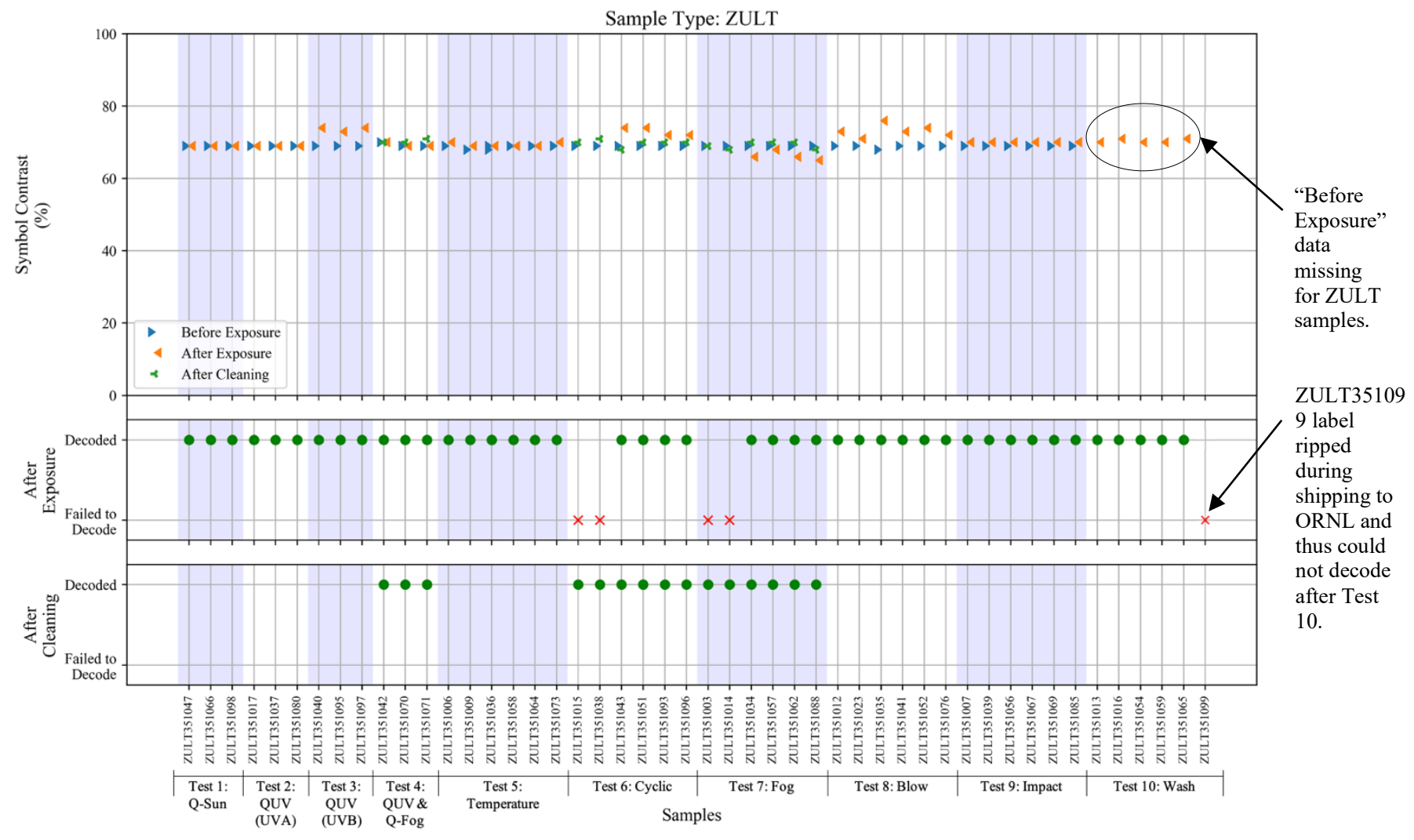

Figure 57. Symbol contrast for Zebra Z-Ultimate 3000T polyester label (ZULT) barcode samples before and after all environmental tests.

Similar to the ZEND samples, the ZULT samples also showed a consistent symbol contrast across all environmental tests. Although a portion of samples failed to decode when exposed to the corrosion tests ( 2 of the 6 samples in Test 6 and 2 of the 6 samples in Test 7), all 12 samples in Tests 6 and 7 decoded after hand cleaning, and the resulting increase in symbol contrast demonstrates that the initial decrease after-exposure comes from the coupon rust dust, not from the sample integrity. A single sample (ZULT351099) exposed to high pressure and temperature water jet testing failed to decode. The sample was presumably damaged during shipping as a puncture was found in the sample's protective foam sleeve when it was unpacked after testing. Note, all 48 ZULT sample identification numbers could be manually read by an ORNL researcher both before and after hand cleaning, regardless of the sample's ability to decode.

\subsection{SUMMARY OF RESULTS BY SAMPLE TYPE}

Table 5 summarizes how the contrast from each sample changed after exposure or after subsequent cleaning. This summary very clearly indicates that on average CERM and ZEND samples exhibited increased contrast after exposure, whereas the other samples exhibited reduced contrast. Table 6 through Table 12 describe the contrast each sample type exhibited as a result of each test. The Final Difference column is shown with Excel's conditional formatting green-white-red color scale to help emphasize the relative magnitude of change. Green signifies the contrast improved the most, and red signifies the contrast was reduced the most relative to the original contrast. 
Table 5. Summary of contrast values for each sample type before exposure, after exposure, and after cleaning. Sorted from largest contrast decline to smallest.

\begin{tabular}{ccccccc}
\hline Sample Type & $\begin{array}{c}\text { Before } \\
\text { Exposure }\end{array}$ & $\begin{array}{c}\text { After } \\
\text { Exposure }\end{array}$ & $\begin{array}{c}\text { After } \\
\text { Cleaning }\end{array}$ & $\begin{array}{c}\text { Difference } \\
\text { After } \\
\text { Exposure }\end{array}$ & $\begin{array}{c}\text { Difference } \\
\text { After } \\
\text { Cleaning }\end{array}$ & $\begin{array}{c}\text { Final } \\
\text { Difference }\end{array}$ \\
\hline CERM & 84.18 & 81.61 & 84.97 & $(2.56)$ & - & 1.66 \\
ZEND & 69.02 & 69.08 & 69.64 & 0.07 & - & 0.98 \\
ZULT & 71.90 & 70.32 & 69.78 & $(1.59)$ & - & $(1.51)$ \\
TESA & 94.66 & 91.48 & 93.17 & $(3.19)$ & - & $(1.81)$ \\
REBO & 72.06 & 69.09 & 70.78 & $(2.97)$ & - & $(2.30)$ \\
ETCH & 96.03 & 91.11 & 96.03 & $(4.92)$ & - & $(2.77)$ \\
YAGL & 70.91 & 66.32 & 65.41 & $(4.59)$ & - & $(3.25)$ \\
\hline
\end{tabular}

Table 6. Contrast values for CERM samples before exposure, after exposure, and after cleaning for each test.

\begin{tabular}{ccccccc}
\hline Test & $\begin{array}{c}\text { Before } \\
\text { Exposure }\end{array}$ & $\begin{array}{c}\text { After } \\
\text { Exposure }\end{array}$ & $\begin{array}{c}\text { After } \\
\text { Cleaning }\end{array}$ & $\begin{array}{c}\text { Difference } \\
\text { After } \\
\text { Exposure }\end{array}$ & $\begin{array}{c}\text { Difference } \\
\text { After } \\
\text { Cleaning }\end{array}$ & $\begin{array}{c}\text { Final } \\
\text { Difference }\end{array}$ \\
\hline CERM & $\mathbf{8 4 . 1 8}$ & $\mathbf{8 1 . 6 1}$ & $\mathbf{8 4 . 9 7}$ & $\mathbf{( 2 . 5 6 )}$ & - & $\mathbf{1 . 6 6}$ \\
\hline 1 - Q-Sun UV & 84.75 & 90.25 & - & 5.50 & - & 5.50 \\
2 - QUV (UVA) & 84.31 & 88.75 & - & 4.44 & - & 4.44 \\
3 - QUV (UVB) & 83.19 & 89.75 & - & 6.56 & - & 6.56 \\
4 - QUV \& Q-FOG & 85.40 & 88.50 & 91.25 & 3.10 & 5.85 & 5.85 \\
5 - Temp & 84.19 & 82.92 & - & $(1.27)$ & - & $(1.27)$ \\
6 - Cyclic & 83.67 & 60.22 & 83.93 & $(23.45)$ & 0.26 & 0.26 \\
7 - Fog & 84.43 & 64.00 & 79.73 & $(20.43)$ & $(4.70)$ & $(4.70)$ \\
8 - Blow & 84.07 & 83.91 & - & $(0.16)$ & - & $(0.16)$ \\
9 - Impact & 82.68 & 81.64 & - & $(1.05)$ & - & $(1.05)$ \\
10 - Wash & 85.06 & 86.18 & - & 1.12 & - & 1.12 \\
\hline
\end{tabular}


Table 7. Contrast values for ZEND samples before exposure, after exposure and after cleaning for each test.

\begin{tabular}{ccccccc}
\hline Test & $\begin{array}{c}\text { Before } \\
\text { Exposure }\end{array}$ & $\begin{array}{c}\text { After } \\
\text { Exposure }\end{array}$ & $\begin{array}{c}\text { After } \\
\text { Cleaning }\end{array}$ & $\begin{array}{c}\text { Difference } \\
\text { After } \\
\text { Exposure }\end{array}$ & $\begin{array}{c}\text { Difference } \\
\text { After } \\
\text { Cleaning }\end{array}$ & $\begin{array}{c}\text { Final } \\
\text { Difference }\end{array}$ \\
\hline ZEND & $\mathbf{6 9 . 0 2}$ & $\mathbf{6 9 . 0 8}$ & $\mathbf{6 9 . 6 4}$ & $\mathbf{0 . 0 7}$ & - & $\mathbf{0 . 9 8}$ \\
\hline 1 - Q-Sun UV & 69.00 & 69.67 & - & 0.67 & - & 0.67 \\
2 - QUV (UVA) & 69.00 & 69.00 & - & - & - & - \\
3 - QUV (UVB) & 69.17 & 70.67 & - & 1.50 & - & 1.50 \\
4 - QUV \& Q-FOG & 69.00 & 69.33 & 70.00 & 0.33 & 1.00 & 1.00 \\
5 - Temp & 69.00 & 69.67 & - & 0.67 & - & 0.67 \\
6 - Cyclic & 69.00 & 70.50 & 70.25 & 1.50 & 1.25 & 1.25 \\
7 - Fog & 69.00 & 60.00 & 68.67 & $(9.00)$ & $(0.33)$ & $(0.33)$ \\
8 - Blow & 69.00 & 70.67 & - & 1.67 & - & 1.67 \\
9 - Impact & 69.00 & 71.00 & - & 2.00 & - & 2.00 \\
10 - Wash & 69.00 & 70.33 & - & 1.33 & - & 1.33 \\
\hline
\end{tabular}

Table 8. Contrast values for ZULT samples before exposure, after exposure and after cleaning for each test.

\begin{tabular}{ccccccc}
\hline Test & $\begin{array}{c}\text { Before } \\
\text { Exposure* }\end{array}$ & $\begin{array}{c}\text { After } \\
\text { Exposure }\end{array}$ & $\begin{array}{c}\text { After } \\
\text { Cleaning }\end{array}$ & $\begin{array}{c}\text { Difference } \\
\text { After } \\
\text { Exposure }\end{array}$ & $\begin{array}{c}\text { Difference } \\
\text { After } \\
\text { Cleaning }\end{array}$ & $\begin{array}{c}\text { Final } \\
\text { Difference }\end{array}$ \\
\hline ZULT & $\mathbf{7 1 . 9 0}$ & $\mathbf{7 0 . 3 2}$ & $\mathbf{6 9 . 7 8}$ & $\mathbf{( 1 . 5 9 )}$ & - & $\mathbf{( 1 . 5 1 )}$ \\
\hline 1 - Q-Sun UV & 71.83 & 69.00 & - & $(2.83)$ & - & $(2.83)$ \\
2 - QUV (UVA) & 72.17 & 69.00 & - & $(3.17)$ & - & $(3.17)$ \\
3 - QUV (UVB) & 71.83 & 73.67 & - & 1.83 & - & 1.83 \\
4 - QUV \& Q-FOG & 72.17 & 69.33 & 70.33 & $(2.83)$ & $(1.83)$ & $(1.83)$ \\
5 - Temp & 71.53 & 69.33 & - & $(2.19)$ & - & $(2.19)$ \\
6 - Cyclic & 71.78 & 73.00 & 69.83 & 1.22 & $(1.94)$ & $(1.94)$ \\
7 - Fog & 72.00 & 66.25 & 69.17 & $(5.75)$ & $(2.83)$ & $(2.83)$ \\
8 - Blow & 72.08 & 73.17 & - & 1.08 & - & 1.08 \\
9 - Impact & 71.75 & 70.00 & - & $(1.75)$ & - & $(1.75)$ \\
10 - Wash & 71.90 & 70.40 & - & $(1.50)$ & - & $(1.50)$ \\
\hline
\end{tabular}

* The before-exposure value for the wash test is highlighted in yellow to help acknowledge that the authors failed to find the before-exposure scans for the ZULT samples subjected to the wash test. To support subsequent quantitative analysis the authors used the average from the before exposure for the other tests as the baseline average value for the pressure wash test. 
Table 9. Contrast values for TESA samples before exposure, after exposure, and after cleaning for each test.

\begin{tabular}{ccccccc}
\hline Test & $\begin{array}{c}\text { Before } \\
\text { Exposure }\end{array}$ & $\begin{array}{c}\text { After } \\
\text { Exposure }\end{array}$ & $\begin{array}{c}\text { After } \\
\text { Cleaning }\end{array}$ & $\begin{array}{c}\text { Difference } \\
\text { After } \\
\text { Exposure }\end{array}$ & $\begin{array}{c}\text { Difference } \\
\text { After } \\
\text { Cleaning }\end{array}$ & $\begin{array}{c}\text { Final } \\
\text { Difference }\end{array}$ \\
\hline TESA & $\mathbf{9 4 . 6 6}$ & $\mathbf{9 1 . 4 8}$ & $\mathbf{9 3 . 1 7}$ & $\mathbf{( 3 . 1 9 )}$ & - & $\mathbf{( 1 . 8 1 )}$ \\
\hline 1 - Q-Sun UV & 94.67 & 92.67 & - & $(2.00)$ & - & $(2.00)$ \\
2 - QUV (UVA) & 94.75 & 92.00 & - & $(2.75)$ & - & $(2.75)$ \\
3 - QUV (UVB) & 94.75 & 89.67 & - & $(5.08)$ & - & $(5.08)$ \\
4 - QUV \& Q-FOG & 94.75 & 91.67 & 93.33 & $(3.08)$ & $(1.42)$ & $(1.42)$ \\
5 - Temp & 94.55 & 94.17 & - & $(0.38)$ & - & $(0.38)$ \\
6 - Cyclic & 94.63 & 89.83 & 93.50 & $(4.80)$ & $(1.13)$ & $(1.13)$ \\
7 - Fog & 94.79 & 84.25 & 92.67 & $(10.54)$ & $(2.13)$ & $(2.13)$ \\
8 - Blow & 94.60 & 92.50 & - & $(2.10)$ & - & $(2.10)$ \\
9 - Impact & 94.54 & 94.00 & - & $(0.54)$ & - & $(0.54)$ \\
10 - Wash & 94.60 & 94.00 & - & $(0.60)$ & - & $(0.60)$ \\
\hline
\end{tabular}

Table 10. Contrast values for REBO samples before exposure, after exposure, and after cleaning for each test.

\begin{tabular}{ccccccc}
\hline Test & $\begin{array}{c}\text { Before } \\
\text { Exposure* }\end{array}$ & $\begin{array}{c}\text { After } \\
\text { Exposure }\end{array}$ & $\begin{array}{c}\text { After } \\
\text { Cleaning }\end{array}$ & $\begin{array}{c}\text { Difference } \\
\text { After } \\
\text { Exposure }\end{array}$ & $\begin{array}{c}\text { Difference } \\
\text { After } \\
\text { Cleaning }\end{array}$ & $\begin{array}{c}\text { Final } \\
\text { Difference }\end{array}$ \\
\hline REBO & $\mathbf{7 2 . 0 6}$ & $\mathbf{6 9 . 0 9}$ & $\mathbf{7 0 . 7 8}$ & $\mathbf{( 2 . 9 7 )}$ & - & $\mathbf{( 2 . 3 0 )}$ \\
\hline 1 - Q-Sun UV & 72.00 & 72.00 & - & - & - & - \\
2 - QUV (UVA) & 72.33 & 72.00 & - & $(0.33)$ & - & $(0.33)$ \\
3 - QUV (UVB) & 72.33 & 73.00 & - & 0.67 & - & 0.67 \\
4 - QUV \& Q-FOG & 72.00 & 71.67 & 72.33 & $(0.33)$ & 0.33 & 0.33 \\
5 - Temp & 71.58 & 48.83 & - & $(22.75)$ & - & $(22.75)$ \\
6 - Cyclic & 72.33 & 70.67 & 70.17 & $(1.67)$ & $(2.17)$ & $(2.17)$ \\
7 - Fog & 72.00 & 63.25 & 69.83 & $(8.75)$ & $(2.17)$ & $(2.17)$ \\
8 - Blow & 72.00 & 73.17 & - & 1.17 & - & 1.17 \\
9 - Impact & 72.00 & 73.50 & - & 1.50 & - & 1.50 \\
10 - Wash & 72.06 & 72.83 & - & 0.77 & - & 0.77 \\
\hline
\end{tabular}

* The before-exposure value for the Wash test is highlighted in yellow to help acknowledge that the authors failed to find the before-exposure scans for the REBO samples subjected to the wash test. To support subsequent quantitative analysis the authors used the average from the before exposure for the other tests as the baseline average value for the pressure wash test. 
Table 11. Contrast values for ETCH samples before exposure, after exposure, and after cleaning for each test.

\begin{tabular}{ccccccc}
\hline Test & $\begin{array}{c}\text { Before } \\
\text { Exposure }\end{array}$ & $\begin{array}{c}\text { After } \\
\text { Exposure }\end{array}$ & $\begin{array}{c}\text { After } \\
\text { Cleaning }\end{array}$ & $\begin{array}{c}\text { Difference } \\
\text { After } \\
\text { Exposure }\end{array}$ & $\begin{array}{c}\text { Difference } \\
\text { After } \\
\text { Cleaning }\end{array}$ & $\begin{array}{c}\text { Final } \\
\text { Difference }\end{array}$ \\
\hline ETCH & $\mathbf{9 6 . 0 3}$ & $\mathbf{9 1 . 1 1}$ & $\mathbf{9 6 . 0 3}$ & $\mathbf{( 4 . 9 2 )}$ & - & $\mathbf{( 2 . 7 7 )}$ \\
\hline 1 - Q-Sun UV & 95.92 & 89.00 & - & $(6.92)$ & - & $(6.92)$ \\
2 - QUV (UVA) & 96.19 & 83.50 & - & $(12.69)$ & - & $(12.69)$ \\
3 - QUV (UVB) & 96.19 & 88.00 & - & $(8.19)$ & - & $(8.19)$ \\
4 - QUV \& Q-FOG & 96.19 & 90.00 & 96.75 & $(6.19)$ & 0.56 & 0.56 \\
5 - Temp & 95.91 & 96.58 & - & 0.67 & - & 0.67 \\
6 - Cyclic & 95.97 & 93.46 & 96.77 & $(2.51)$ & 0.79 & 0.79 \\
7 - Fog & 95.96 & 83.14 & 94.58 & $(12.82)$ & $(1.38)$ & $(1.38)$ \\
8 - Blow & 95.94 & 95.25 & - & $(0.69)$ & - & $(0.69)$ \\
9 - Impact & 96.01 & 96.00 & - & $(0.01)$ & - & $(0.01)$ \\
10 - Wash & 96.00 & 96.17 & - & 0.17 & - & 0.17 \\
\hline
\end{tabular}

Table 12. Contrast values for YAGL samples before exposure, after exposure, and after cleaning for each test.

\begin{tabular}{ccccccc}
\hline Test & $\begin{array}{c}\text { Before } \\
\text { Exposure }\end{array}$ & $\begin{array}{c}\text { After } \\
\text { Exposure }\end{array}$ & $\begin{array}{c}\text { After } \\
\text { Cleaning }\end{array}$ & $\begin{array}{c}\text { Difference } \\
\text { After } \\
\text { Exposure }\end{array}$ & $\begin{array}{c}\text { Difference } \\
\text { After } \\
\text { Cleaning }\end{array}$ & $\begin{array}{c}\text { Final } \\
\text { Difference }\end{array}$ \\
\hline YAGL & $\mathbf{7 0 . 9 1}$ & $\mathbf{6 6 . 3 2}$ & $\mathbf{6 5 . 4 1}$ & $\mathbf{( 4 . 5 9 )}$ & - & $\mathbf{( 3 . 2 5 )}$ \\
\hline 1 - Q-Sun UV & 70.67 & 68.00 & - & $(2.67)$ & - & $(2.67)$ \\
2 - QUV (UVA) & 71.48 & 69.88 & - & $(1.60)$ & - & $(1.60)$ \\
3 - QUV (UVB) & 70.94 & 69.25 & - & $(1.69)$ & - & $(1.69)$ \\
4 - QUV \& Q-FOG & 71.75 & 52.67 & 56.00 & $(19.08)$ & $(15.75)$ & $(15.75)$ \\
5 - Temp & 70.27 & 69.83 & - & $(0.44)$ & - & $(0.44)$ \\
6 - Cyclic & 71.38 & 68.92 & 72.68 & $(2.46)$ & 1.30 & 1.30 \\
7 - Fog & 70.67 & 61.17 & 67.55 & $(9.50)$ & $(3.13)$ & $(3.13)$ \\
8 - Blow & 70.59 & 67.31 & - & $(3.28)$ & - & $(3.28)$ \\
9 - Impact & 70.47 & 68.67 & - & $(1.80)$ & - & $(1.80)$ \\
10 - Wash & 70.87 & 67.46 & - & $(3.40)$ & - & $(3.40)$ \\
\hline
\end{tabular}

\section{CONSIDERATIONS FOR FUTURE WORK}

Bare A516 steel surfaces should be painted after the barcode sample is attached. Rust surface residue on many of the sample's caused the verifier to fail to decode barcodes subjected to some tests; however, we believe the observed rust is from the degradation of the bare A516 coupon and not the stainless-steel or label-type samples. The A516 steel used for 30B and $48 \mathrm{Y}$ cylinders is painted during fabrication and would not be exposed throughout the remainder of a cylinder's life.

Although the ORNL team expected the untreated surfaces of the A516 steel coupons to rust, the team did not anticipate the rust to transfer to the surfaces of the stainless-steel or label-type samples. This behavior was exceptionally apparent for the samples that underwent QUV and Q-FOG testing (Test 4), cyclic corrosion testing (Test 6), and corrosion testing (Test 7). It did occur to a lesser degree in the samples that 
were subjected to the UVA and UVB testing (Tests 2 and 3). The authors believe that the rust from the coupons spread onto the barcode from circulating moist air in the test chambers. All but two of the affected samples decoded after a team member cleaned the surface with Formula 409 and WD-40.

Adding a guide to the barcode verifier sped up the team's ability to position barcodes in the verifier's field of view; however, it caused the verifier to fail to decode a few samples. Our team attached an optical breadboard with a grid of tapped holes to the barcode verifier platform. This grid of tapped holes allowed the team to position guide rails to accelerate and standardize positioning barcodes in the verifier's field of view. The optical breadboard and guide rails can be seen in Figure 58. For some barcodes, the verifier initially failed to decode and verify the barcode at the location prescribed by the guides. However, if the sample was shifted slightly or rotated, the barcode could be verified. The team has not observed difficulty decoding 2D barcodes using handheld barcode scanners and believe this behavior is a result of where in the barcode image the verification algorithm tries to start.

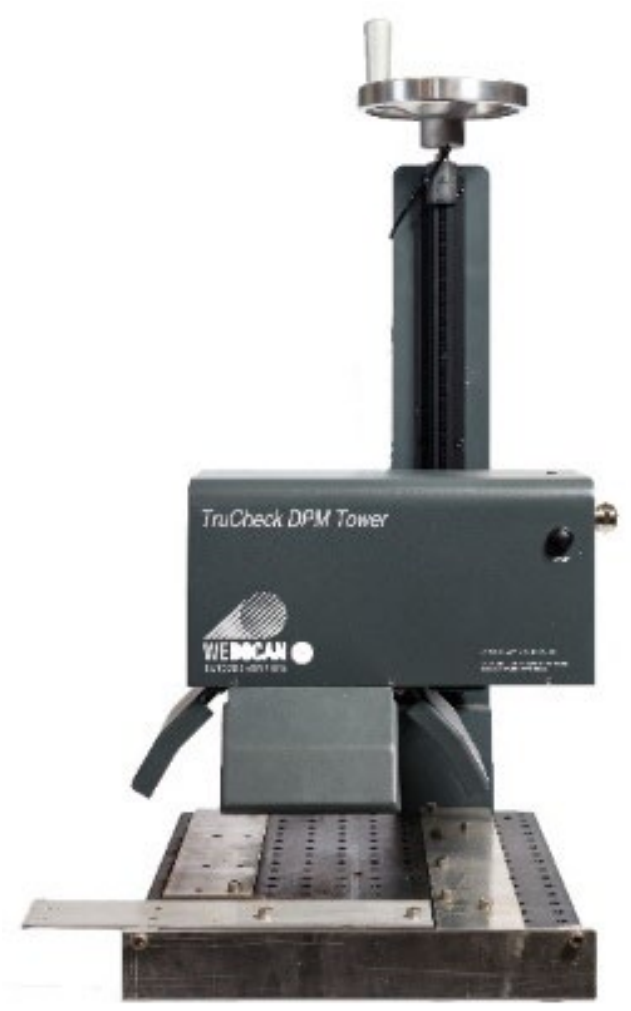

Figure 58. Webscan TruCheck DPM Tower with optical breadboard and sample guides attached.

\section{DISCUSSION}

Under limited-scope environmental testing of 444 samples involving 7 barcode marking techniques, all samples performed relatively equally. However, each sample group has its own strengths and weaknesses. Regardless of the marking technique, all 2D barcodes were successfully decoded after 7 of the 10 environmental tests. Some of the tests caused a decrease in measured cell or symbol contrast, but the exposure was not enough to prevent the barcodes from decoding. All sample types experienced at least one failure to decode after corrosion (salt fog) testing (Test 7), and some sample types experienced at least one failure to decode after QUV and salt fog testing (Test 4) and cyclic corrosion testing (Test 6). After a simple hand cleaning process, all except for three of these samples were successfully decoded. Of these three samples, one (ZULT351099) is suspected to have ripped during shipping, although it was not 
immediately clear why the other two samples (YAGL123476 and YAGL123486) did not decode. Regardless of the sample's ability to decode postexposure, all sample identification numbers could be manually read by an ORNL researcher both before and after hand cleaning.

Additionally, the coupon attachment method did not appear to influence the sample's ability to decode, and there was not a clear advantage for the use of one sample type (stainless-steel or label-type) over the other. Anecdotal evidence suggests that chemically etched and paint filled markings and epoxy adhesives fail over time, but this testing campaign did not reveal evidence to support these observations. Any of the label-type samples (REBO, TESA, ZEND, and ZULT) could provide an easy to apply solution as a global identifier label that supplements current identification plates on $\mathrm{UF}_{6}$ cylinders in circulation before they are due for recertification.

The highest contrast readings across all environmental tests were demonstrated by ETCH (stainless-steel type) and TESA (label-type) samples, but the ZEND and ZULT label-type samples had the most consistent contrast readings before and after environmental exposure. Overall, each of the seven barcode marking techniques are reasonable candidates for use in the field following limited-scope environmental testing. A summary of the advantages and weaknesses for each sample type is shown in Table 13.

Table 13. Strengths and weaknesses of sample types exposed to environmental testing.

\begin{tabular}{|c|c|c|c|}
\hline $\begin{array}{l}\text { Sample } \\
\text { Type }\end{array}$ & $\begin{array}{l}\text { Stainless-Steel or } \\
\text { Label-Type }\end{array}$ & Advantage & Weakness \\
\hline CERM & Stainless-Steel & High Cell Contrast & Corrosion Tests \\
\hline ETCH & Stainless-Steel & High Cell Contrast & UV Tests (all) \\
\hline YAGL & Stainless-Steel & None & $\begin{array}{l}\text { Corrosion Tests and Moderate Cell } \\
\text { Contrast }\end{array}$ \\
\hline REBO & Label-Type & Symbol Contrast Consistency & $\begin{array}{l}\text { Temperature Tests and Moderate } \\
\text { Symbol Contrast }\end{array}$ \\
\hline TESA & Label-Type & High Cell Contrast and Consistency & UVB Tests \\
\hline ZEND & Label-Type & Symbol Contrast Consistency & Moderate Symbol Contrast \\
\hline ZULT & Label-Type & Symbol Contrast Consistency & Moderate Symbol Contrast \\
\hline
\end{tabular}

${ }^{1}$ High Cell or Symbol Contrast: $80 \%$ or above Moderate Cell or Symbol Contrast: $60 \%-80 \%$ Low Cell or Symbol Contrast: $60 \%$ or below

Generally, the label-type samples had a smaller decrease in symbol contrast after environmental exposure.

Overall, the ZEND and ZULT sample sets demonstrated the most consistent symbol contrast for all 10 environmental tests. Each remaining sample set was susceptible to at least one type of environmental exposure. Although all REBO labels decoded after the temperature testing, the label discoloration was unique to this sample set and caused a significant decrease in symbol contrast. Similarly, the ETCH samples performed poorly for all UV based tests when compared to other stainless-steel samples. Additionally, it appeared that the TESA tape samples were susceptible to UVB exposure, but they did not show a similar decrease in cell contrast for other UV tests. Despite their susceptibility to the abovementioned exposures, the ETCH and TESA samples had the highest overall contrast values compared to the other sample types.

Any coupon rust presented issues for all sample types. Specifically, many CERM and YAGL samples failed to decode after corrosion tests (Tests 6 and 7). Fewer than half of the samples from each set could be decoded before hand cleaning. After hand cleaning, the YAGL sample set consistently preformed more 
poorly than the others. It would have been more representative to paint or otherwise treat the bare A516 to better represent the conditions of cylinders in the field.

\section{CONCLUSIONS}

The postexposure results described in this report suggest that of the sample types tested, ball-blasted stainless-steel sheets laser marked with CerMark laser marking ink (CERM) would be the most robust material and marking technique for $\mathrm{UF}_{6}$ supplemental cylinder identifiers. Although stainless-steel supplemental cylinder identifiers could be welded or affixed using epoxies such as the Aremco 517 epoxy, thermal printer-based labels like the Zebra Z-Ultimate 3000T (ZULT) offer an alternative that are extremely easy to affix to a surface, very cost effective to produce on-site (suitable industrial label printers cost about $\$ 1,500$ and consumables cost about $\$ 0.10$ per label) and were nearly as robust as the top performing CERM samples. Although ZEND (Z-Endure 4000T) slightly outperformed the ZULT labels and the manufacturer rates them for 10-year use outside compared to 3 years for the Z-Ultimate 3000T labels, Z-Endure 4000T are special order and exhibited the printing flaws described in Section 2. Table 14 summarizes our conclusion for all the sample types.

Table 14. Summary of samples tested and conclusions based on quantitative and qualitative analysis of the results.

\begin{tabular}{|c|c|c|c|}
\hline $\begin{array}{l}\text { Sample } \\
\text { Type }\end{array}$ & Conclusion & Sample Description & Conclusion Explanation \\
\hline CERM & $\begin{array}{l}\text { Highly } \\
\text { recommended }\end{array}$ & $\begin{array}{l}\text { Ball-blasted stainless-steel } \\
\text { sheets laser marked with } \\
\text { CerMark laser marking ink }\end{array}$ & $\begin{array}{l}\text { Best average contrast change. Contrast increased } \\
\text { after UV exposure tests (Tests } 1,2,3 \text {, and } 4 \text { ) and } \\
\text { only decreased slightly for salt fog (Test } 7) \text {. }\end{array}$ \\
\hline ZULT & $\begin{array}{l}\text { Recommended } \\
\text { label alternative } \\
\text { to stainless steel } \\
\end{array}$ & $\begin{array}{l}\text { Zebra Z-Ultimate } 3000 \mathrm{~T} \\
\text { polyester labels }\end{array}$ & $\begin{array}{l}\text { Affordable, widely available stainless steel } \\
\text { alternative label material that performed nearly as } \\
\text { well as the ZEND }\end{array}$ \\
\hline ZEND & $\begin{array}{l}\text { Recommended } \\
\text { label alternative } \\
\text { to stainless steel }\end{array}$ & $\begin{array}{l}\text { Zebra Z-Endure 4000T acrylic } \\
\text { labels }\end{array}$ & $\begin{array}{l}\text { May be challenging to procure but may offer more } \\
\text { robustness than other labels. }\end{array}$ \\
\hline TESA & Acceptable & Tesa Secure 6973 tape & $\begin{array}{l}\text { Appears to offer little benefit compared to more } \\
\text { widely available polyester or acrylic labels and } \\
\text { requires more complicated and expensive marking } \\
\text { system. }\end{array}$ \\
\hline ETCH & Acceptable & $\begin{array}{l}\text { Ball-blasted stainless-steel } \\
\text { sheets chemically etched with } \\
\text { etched areas filled with paint }\end{array}$ & $\begin{array}{l}\text { Appears to offer little benefit compared to more } \\
\text { widely available polyester or acrylic labels and } \\
\text { requires more complicated and expensive marking } \\
\text { system. }\end{array}$ \\
\hline REBO & $\begin{array}{c}\text { Not } \\
\text { recommended }\end{array}$ & $\begin{array}{l}\text { Rebo ST700 Premium } \\
\text { Industrial Vinyl }\end{array}$ & Discolored considerably during temperature testing. \\
\hline YAGL & $\begin{array}{c}\text { Not } \\
\text { recommended }\end{array}$ & $\begin{array}{l}\text { Ball-blasted stainless-steel } \\
\text { sheets marked with YAG laser }\end{array}$ & $\begin{array}{l}\text { Contrast decreased significantly during QUV and } \\
\text { Q-FOG test (Test 4), but some samples subjected to } \\
\text { salt fog (Test 7) remained undecodable even after } \\
\text { cleaning. }\end{array}$ \\
\hline
\end{tabular}




\section{ACKNOWLEDGMENTS}

Funding for this project was provided by the Department of Energy's NNSA Office of International Nuclear Safeguards (OINS). The authors would also like to thank Scott Palko for his assistance preparing samples for testing and Ed Wonder (Ayr Hill Group LLC, a subcontractor to Brookhaven National Laboratory) for his assistance refining the test plan. 


\section{REFERENCES}

“"

[1] J. Garner and N. Pratt, "Design Specifications for a Machine-Readable Container Identifier," presented at the 58th Annual Meeting of the Institute of Nuclear Materials Management (INMM 2017), Indian Wells, CA, USA, 2017.

[2] UF 6 Cylinder Identification, World Nuclear Transport Institute, London, United Kingdon, 2017. [Online]. Available: https://www.wnti.co.uk/media/87140/WNTI\%20STANDARD\%20\%20UF6\%20Cylinder\%20Identification\%20-\%20Version\%20--\%20Final\%20-\%202017.pdf

[3] ISO/IEC 16022 - Information technology - Automatic identification and data capture techniques - Data Matrix bar code symbology specification, 16022:2006, ISO, 2006. [Online]. Available: https://www.iso.org/obp/ui/\#iso:std:iso-iec:16022:ed-2:v1:en

[4] ISO/IEC 18004 - Information technology - Automatic identification and data capture techniques QR Code bar code symbology specification, ISO/IEC 18004:2015, ISO, 2015. [Online].

Available: https://www.iso.org/obp/ui/\#iso:std:iso-iec:18004:ed-3:v1:en

[5] ISO/IEC 15415 - Information technology - Automatic identification and data capture techniques Bar code symbol print quality test specification - Two-dimensional symbols, ISO/IEC 15415:2011, ISO, 2011. [Online]. Available: https://www.iso.org/obp/ui/\#iso:std:isoiec:15415:ed-2:v1:en

[6] ISO/IEC TR 29158 - Information technology - Automatic identification and data capture techniques - Direct Part Mark (DPM) Quality Guideline, ISO/IEC TR 29158:2011, ISO, 2011. [Online]. Available: https://www.iso.org/obp/ui/\#iso:std:iso-iec:tr:29158:ed-1:v1:en

[7] ID CODE HANDBOOK: 2-D CODE BASIC GUIDE. (2010). Keyence.

[8] tesa. tesa 6973 Laser Label Product Information. (2019). [Online]. Available: http://1.tesa.com/?ip=06973

[9] Z. Technologies. Z-Endure 4000T Materials Specification Sheet. (2018).

[10] Z. Technologies. Z-Ultimate 3000T Specification Sheet.

[11] J. Garner and L. Scott, "Results of Data Matrix Barcode Testing for Field Applications," in 41st ESARDA Annual Meeting Symposium on Safeguards and Nuclear Material Management, Stresa, Italy, 14-16 May 2019, pp. 721-730, doi: 0.2760/159550.

[12] ASTM G155 - Standard Practice for Operating Xenon Arc Light Apparatus for Exposure of NonMetallic Materials, ASTM, 2013.

[13] ASTM G154 - Standard Practice for Operating Fluorescent Ultraviolet (UV) Lamp Apparatus for Exposure of Nonmetallic Materials, ASTM, 2016.

[14] Z. Corp. Zebra 8000T Z-Endure Fact Sheet, Rev. 2. (Dec. 2015).

[15] ASTM D5894 - Standard Practice for Cyclic Salt Fog/UV Exposure of Painted Metal, (Alternating Exposures in a Fog/Dry Cabinet and a UV/Condensation Cabinet), ASTM, 2016.

[16] GMW 14872 - Cyclic Corrosion Laboratory Test - Issue 4; English, G.-G. Motors, Englewood, CO, 2018.

[17] ASTM B117 - Standard Practice for Operating Salt Spray (Fog) Apparatus, ASTM, 2019.

[18] MIL-STD-810G W/CHANGE 1 - Environmental Engineering Considerations and Laboratory Tests, D. o. Defense, 4/15/2014 2014. 
[19] IEC 61010-1 - Safety requirements for electrical equipment for measurement, control, and laboratory use, IEC, 2017.

[20] IEC 60529 CORR 1 - Degrees of protection provided by enclosures (IP Code), IEC, 2013. 
APPENDIX A. EPOXY PREPARATION 



\section{APPENDIX A. EPOXY PREPARATION}

Figures A-1 through A-6 illustrate the process used to epoxy the stainless-steel samples to the A516 coupons. A total of 116 samples were epoxied using this process.

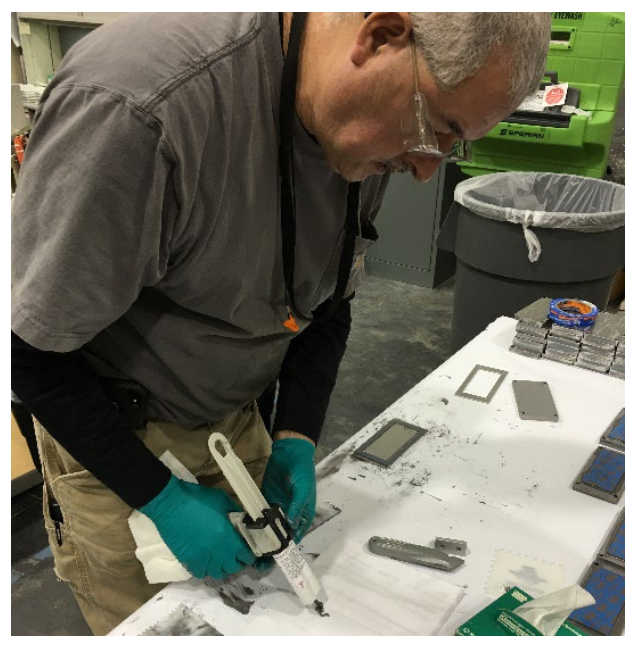

Figure A-1. Epoxy is expelled until both sides are flowing from the tube.

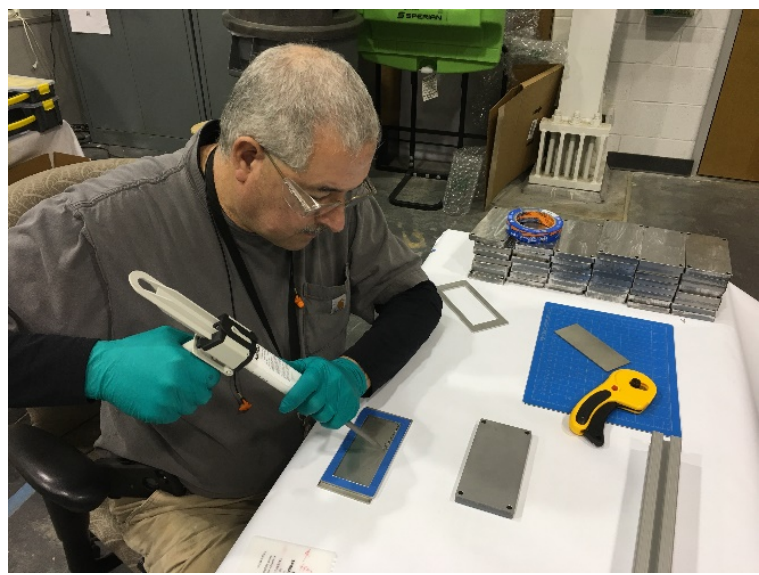

Figure A-3. The stainless-steel barcode sample is positioned in a template cut to the size of the A516 coupon. Epoxy is then expelled onto the back of the sample.

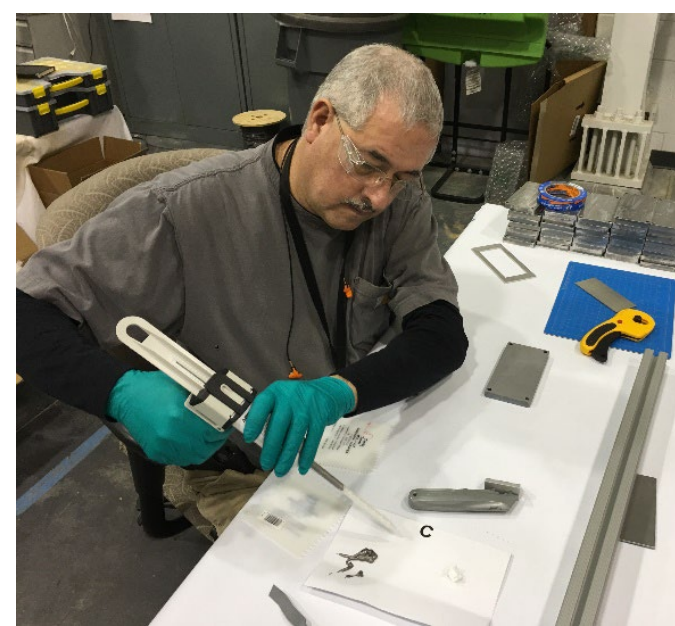

Figure A-2. A nozzle is installed, and the epoxy is expelled until it flows freely from the nozzle.

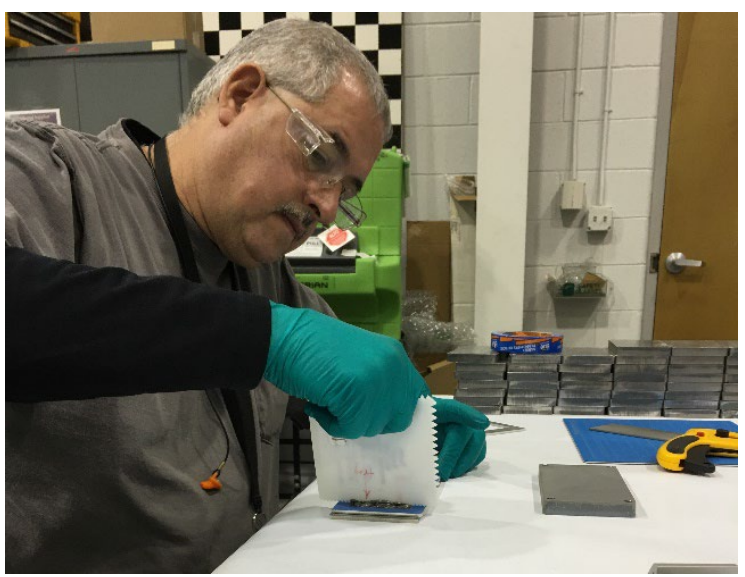

Figure A-4. A disposable notched trowel is used to ensure an even coating of epoxy on the back of the sample. 


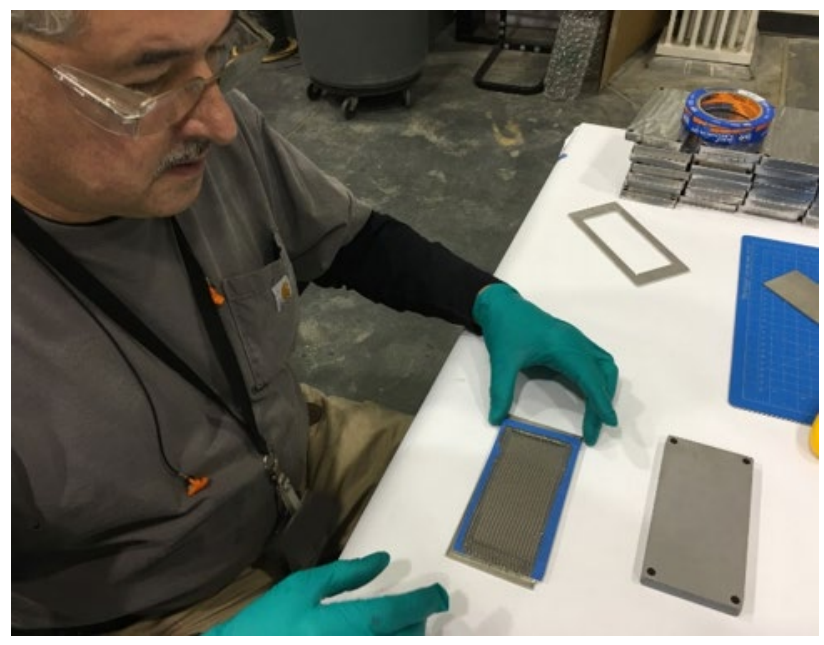

Figure A-5. At this point, the stainless-steel barcode sample has a uniform amount of epoxy distributed across the surface and the technician can flip the A516 coupon onto the sample.

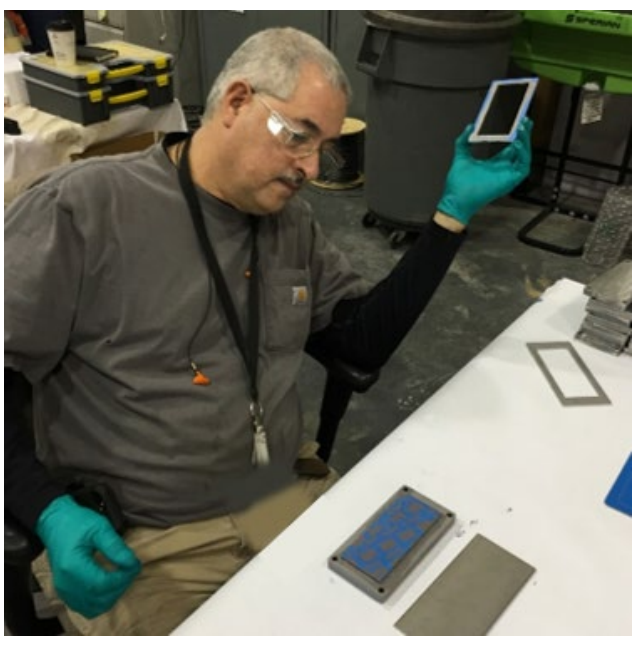

Figure A-6. Once the template (as shown in the technician's left hand) is removed, the stainlesssteel barcode sample is left on the A516 coupon in a consistent position. 
APPENDIX B. BARCODE SAMPLES GROUPED BY SAMPLE TYPE 



\section{APPENDIX B. BARCODE SAMPLES GROUPED BY SAMPLE TYPE}

Tables B-1 through B-7 provide information on each sample with the seven sample types including sample ID, type of environmental test performed, coupon finish, and attachment method.

Table B-1. 87 CerMark-coated laser-marked stainless-steel barcode samples and associated environmental tests.

\begin{tabular}{|c|c|c|c|}
\hline Sample ID & Environmental Test & Coupon Finish & Attachment Method \\
\hline CERM123400 & 5 - Temperature & Free & Free \\
\hline CERM123401 & 10 - Wash & Free & Free \\
\hline CERM123402 & 2 - QUV (UVA) & Polished & Weld \\
\hline CERM123403 & 7 - Corrosion (Fog) & Free & Free \\
\hline CERM123404 & 5 - Temperature & Sandblasted & Aremco 517 Epoxy \\
\hline CERM123405 & 6 - Cyclic & Free & Free \\
\hline CERM123406 & 7 - Corrosion (Fog) & Sandblasted & Aremco 517 Epoxy \\
\hline CERM123407 & 9 - Impact & Free & Free \\
\hline CERM123409 & 8 - Blow & Free & Free \\
\hline CERM123410 & 8 - Blow & Sandblasted & Aremco 517 Epoxy \\
\hline CERM123411 & 6 - Cyclic & Sandblasted & Aremco 517 Epoxy \\
\hline CERM123412 & 7 - Corrosion (Fog) & Free & Free \\
\hline CERM123413 & 1 - Q-SUN & Free & Free \\
\hline CERM123414 & 4 - QUV \& Q-FOG & Polished & Weld \\
\hline CERM123416 & 10 - Wash & Sandblasted & Aremco 517 Epoxy \\
\hline CERM123417 & 5 - Temperature & Free & Free \\
\hline CERM123418 & 8 - Blow & Free & Free \\
\hline CERM123419 & 6 - Cyclic & Polished & Aremco 517 Epoxy \\
\hline CERM123420 & 7 - Corrosion (Fog) & Free & Free \\
\hline CERM123421 & 5 - Temperature & Sandblasted & Aremco 517 Epoxy \\
\hline CERM123422 & 1 - Q-SUN & Polished & Aremco 517 Epoxy \\
\hline CERM123423 & 6 - Cyclic & Free & Free \\
\hline CERM123424 & 8 - Blow & Sandblasted & Aremco 517 Epoxy \\
\hline CERM123425 & 4 - QUV \& Q-FOG & Sandblasted & Aremco 517 Epoxy \\
\hline CERM123426 & 5 - Temperature & Free & Free \\
\hline CERM123427 & 10 - Wash & Free & Free \\
\hline CERM123428 & 9 - Impact & Free & Free \\
\hline CERM123430 & 10 - Wash & Sandblasted & Aremco 517 Epoxy \\
\hline CERM123431 & 6 - Cyclic & Sandblasted & Aremco 517 Epoxy \\
\hline CERM123432 & 2 - QUV (UVA) & Free & Free \\
\hline CERM123433 & 8 - Blow & Sandblasted & Aremco 517 Epoxy \\
\hline CERM123434 & 5 - Temperature & Sandblasted & Aremco 517 Epoxy \\
\hline CERM123435 & 8 - Blow & Free & Free \\
\hline CERM123436 & 3 - QUV (UVB) & Sandblasted & Aremco 517 Epoxy \\
\hline CERM123438 & 3 - QUV (UVB) & Free & Free \\
\hline CERM123440 & 9 - Impact & Sandblasted & Aremco 517 Epoxy \\
\hline CERM123441 & 4 - QUV \& Q-FOG & Free & Free \\
\hline
\end{tabular}


Table B-1. 87 CerMark-coated laser-marked stainless-steel barcode samples and associated environmental tests, continued.

\begin{tabular}{|c|c|c|c|}
\hline Sample ID & Environmental Test & Coupon Finish & Attachment Method \\
\hline CERM123443 & 10 - Wash & Free & Free \\
\hline CERM123444 & 5 - Temperature & Polished & Aremco 517 Epoxy \\
\hline CERM123445 & 10 - Wash & Free & Free \\
\hline CERM123446 & 1 - Q-SUN & Polished & Weld \\
\hline CERM123447 & 8 - Blow & Free & Free \\
\hline CERM123448 & 6 - Cyclic & Sandblasted & Aremco 517 Epoxy \\
\hline CERM123449 & 7 - Corrosion (Fog) & Sandblasted & Aremco 517 Epoxy \\
\hline CERM123450 & 4 - QUV \& Q-FOG & Polished & Aremco 517 Epoxy \\
\hline CERM123451 & 10 - Wash & Sandblasted & Aremco 517 Epoxy \\
\hline CERM123452 & 6 - Cyclic & Polished & Weld \\
\hline CERM123454 & 6 - Cyclic & Polished & Weld \\
\hline CERM123457 & 7 - Corrosion (Fog) & Sandblasted & Aremco 517 Epoxy \\
\hline CERM123458 & 9 - Impact & Free & Free \\
\hline CERM123459 & 6 - Cyclic & Free & Free \\
\hline CERM123460 & 2 - QUV (UVA) & Polished & Aremco 517 Epoxy \\
\hline CERM123461 & 10 - Wash & Polished & Weld \\
\hline CERM123462 & 7 - Corrosion (Fog) & Free & Free \\
\hline CERM123464 & 8 - Blow & Polished & Weld \\
\hline CERM123465 & 3 - QUV (UVB) & Polished & Weld \\
\hline CERM123466 & 9 - Impact & Sandblasted & Aremco 517 Epoxy \\
\hline CERM123467 & 10 - Wash & Free & Free \\
\hline CERM123468 & 5 - Temperature & Free & Free \\
\hline CERM123469 & 8 - Blow & Sandblasted & Aremco 517 Epoxy \\
\hline CERM123470 & 10 - Wash & Polished & Weld \\
\hline CERM123471 & 7 - Corrosion (Fog) & Polished & Weld \\
\hline CERM123472 & 10 - Wash & Sandblasted & Aremco 517 Epoxy \\
\hline CERM123473 & 6 - Cyclic & Sandblasted & Aremco 517 Epoxy \\
\hline CERM123475 & 6 - Cyclic & Sandblasted & Aremco 517 Epoxy \\
\hline CERM123476 & 8 - Blow & Polished & Weld \\
\hline CERM123478 & 5 - Temperature & Polished & Weld \\
\hline CERM123479 & 9 - Impact & Sandblasted & Aremco 517 Epoxy \\
\hline CERM123480 & 7 - Corrosion (Fog) & Polished & Weld \\
\hline CERM123481 & 5 - Temperature & Polished & Aremco 517 Epoxy \\
\hline CERM123482 & 9 - Impact & Sandblasted & Aremco 517 Epoxy \\
\hline CERM123483 & 7 - Corrosion (Fog) & Free & Free \\
\hline CERM123484 & 5 - Temperature & Free & Free \\
\hline CERM123485 & 9 - Impact & Polished & Weld \\
\hline CERM123487 & 8 - Blow & Free & Free \\
\hline CERM123488 & 2 - QUV (UVA) & Sandblasted & Aremco 517 Epoxy \\
\hline CERM123489 & 9 - Impact & Free & Free \\
\hline CERM123490 & 5 - Temperature & Sandblasted & Aremco 517 Epoxy \\
\hline
\end{tabular}


Table B-1. 87 CerMark-coated laser-marked stainless-steel barcode samples and associated environmental tests, continued.

\begin{tabular}{llll}
\hline Sample ID & Environmental Test & Coupon Finish & Attachment Method \\
\hline CERM123491 & 6 - Cyclic & Sandblasted & Aremco 517 Epoxy \\
CERM123492 & 9 - Impact & Free & Free \\
CERM123493 & 9 - Impact & Polished & Weld \\
CERM123494 & 6- Cyclic & Free & Free \\
CERM123495 & 1 - Q-SUN & Sandblasted & Aremco 517 Epoxy \\
CERM123496 & 6- Cyclic & Free & Free \\
CERM123497 & 7 - Corrosion (Fog) & Sandblasted & Aremco 517 Epoxy \\
CERM123498 & 5 - Temperature & Sandblasted & Aremco 517 Epoxy \\
CERM123499 & 3- QUV (UVB) & Polished & Aremco 517 Epoxy \\
\hline
\end{tabular}


Table B-2. 87 chemically etched stainless-steel barcode samples and associated environmental tests.

\begin{tabular}{|c|c|c|c|}
\hline Sample ID & Environmental Test & Coupon Finish & Attachment Methoc \\
\hline ETCH123400 & 9 - Impact & Polished & Weld \\
\hline ETCH123401 & 9 - Impact & Free & Free \\
\hline ETCH123402 & 4 - QUV \& Q-FOG & Polished & Aremco 517 Epoxy \\
\hline ETCH123403 & 9 - Impact & Sandblasted & Aremco 517 Epoxy \\
\hline ETCH123404 & $10-$ Wash & Sandblasted & Aremco 517 Epoxy \\
\hline ETCH123405 & 5 - Temperature & Free & Free \\
\hline ETCH123406 & 4 - QUV \& Q-FOG & Sandblasted & Aremco 517 Epoxy \\
\hline ETCH123407 & 3 - QUV (UVB) & Free & Free \\
\hline ETCH123408 & 8 - Blow & Sandblasted & Aremco 517 Epoxy \\
\hline ETCH123409 & 2 - QUV (UVA) & Polished & Aremco 517 Epoxy \\
\hline ETCH123410 & 6 - Cyclic & Sandblasted & Aremco 517 Epoxy \\
\hline ETCH123411 & 7 - Corrosion (Fog) & Sandblasted & Aremco 517 Epoxy \\
\hline ETCH123412 & 4 - QUV \& Q-FOG & Free & Free \\
\hline ETCH123413 & 8 - Blow & Polished & Weld \\
\hline ETCH123414 & 6 - Cyclic & Free & Free \\
\hline ETCH123415 & 5 - Temperature & Free & Free \\
\hline ETCH123416 & 1 - Q-SUN & Polished & Aremco 517 Epoxy \\
\hline ETCH123417 & 7 - Corrosion (Fog) & Free & Free \\
\hline ETCH123419 & 8 - Blow & Free & Free \\
\hline ETCH123420 & 6 - Cyclic & Sandblasted & Aremco 517 Epoxy \\
\hline ETCH123421 & 7 - Corrosion (Fog) & Free & Free \\
\hline ETCH123422 & 2 - QUV (UVA) & Free & Free \\
\hline ETCH123423 & 7 - Corrosion (Fog) & Sandblasted & Aremco 517 Epoxy \\
\hline ETCH123424 & 9 - Impact & Free & Free \\
\hline ETCH123425 & 7 - Corrosion (Fog) & Sandblasted & Aremco 517 Epoxy \\
\hline ETCH123426 & 5 - Temperature & Sandblasted & Aremco 517 Epoxy \\
\hline ETCH123427 & 6 - Cyclic & Free & Free \\
\hline ETCH123429 & 9 - Impact & Free & Free \\
\hline ETCH123430 & 2 - QUV (UVA) & Sandblasted & Aremco 517 Epoxy \\
\hline ETCH123431 & 4 - QUV \& Q-FOG & Polished & Weld \\
\hline ETCH123434 & 6 - Cyclic & Sandblasted & Aremco 517 Epoxy \\
\hline ETCH123436 & 6 - Cyclic & Polished & Weld \\
\hline ETCH123437 & 3 - QUV (UVB) & Polished & Weld \\
\hline ETCH123438 & 1 - Q-SUN & Sandblasted & Aremco 517 Epoxy \\
\hline ETCH123439 & 9 - Impact & Sandblasted & Aremco 517 Epoxy \\
\hline ETCH123441 & 9 - Impact & Sandblasted & Aremco 517 Epoxy \\
\hline ETCH123442 & 8 - Blow & Sandblasted & Aremco 517 Epoxy \\
\hline ETCH123443 & 7 - Corrosion (Fog) & Sandblasted & Aremco 517 Epoxy \\
\hline ETCH123444 & $10-$ Wash & Free & Free \\
\hline ETCH123445 & 7 - Corrosion (Fog) & Polished & Weld \\
\hline ETCH123446 & 5 - Temperature & Free & Free \\
\hline
\end{tabular}


Table B-2. 87 chemically etched stainless-steel barcode samples and associated environmental tests, continued.

\begin{tabular}{|c|c|c|c|}
\hline Sample ID & Environmental Test & Coupon Finish & Attachment Method \\
\hline ETCH123447 & $10-$ Wash & Free & Free \\
\hline ETCH123448 & 6 - Cyclic & Polished & Aremco 517 Epoxy \\
\hline ETCH123449 & 10 - Wash & Sandblasted & Aremco 517 Epoxy \\
\hline ETCH123450 & 6 - Cyclic & Free & Free \\
\hline ETCH123451 & $10-$ Wash & Free & Free \\
\hline ETCH123452 & 10 - Wash & Free & Free \\
\hline ETCH123454 & 5 - Temperature & Sandblasted & Aremco 517 Epoxy \\
\hline ETCH123457 & 8 - Blow & Free & Free \\
\hline ETCH123458 & 7 - Corrosion (Fog) & Polished & Weld \\
\hline ETCH123459 & 10 - Wash & Polished & Weld \\
\hline ETCH123460 & 8 - Blow & Free & Free \\
\hline ETCH123461 & $10-$ Wash & Free & Free \\
\hline ETCH123463 & 9 - Impact & Free & Free \\
\hline ETCH123464 & 6 - Cyclic & Free & Free \\
\hline ETCH123466 & 9 - Impact & Polished & Weld \\
\hline ETCH123467 & $10-$ Wash & Polished & Weld \\
\hline ETCH123468 & $10-$ Wash & Sandblasted & Aremco 517 Epoxy \\
\hline ETCH123469 & 6 - Cyclic & Sandblasted & Aremco 517 Epoxy \\
\hline ETCH123470 & 9 - Impact & Free & Free \\
\hline ETCH123471 & 7 - Corrosion (Fog) & Sandblasted & Aremco 517 Epoxy \\
\hline ETCH123472 & 5 - Temperature & Free & Free \\
\hline ETCH123473 & 2 - QUV (UVA) & Polished & Weld \\
\hline ETCH123474 & 10 - Wash & Sandblasted & Aremco 517 Epoxy \\
\hline ETCH123475 & 8 - Blow & Sandblasted & Aremco 517 Epoxy \\
\hline ETCH123476 & 7 - Corrosion (Fog) & Free & Free \\
\hline ЕTCH123477 & 5 - Temperature & Polished & Weld \\
\hline ETCH123478 & 6 - Cyclic & Polished & Weld \\
\hline ETCH123479 & 8 - Blow & Sandblasted & Aremco 517 Epoxy \\
\hline ETCH123480 & 1 - Q-SUN & Polished & Weld \\
\hline ETCH123481 & 5 - Temperature & Free & Free \\
\hline ETCH123482 & 10 - Wash & Sandblasted & Aremco 517 Epoxy \\
\hline ETCH123483 & 5 - Temperature & Sandblasted & Aremco 517 Epoxy \\
\hline ETCH123484 & 7 - Corrosion (Fog) & Free & Free \\
\hline ETCH123485 & 5 - Temperature & Polished & Aremco 517 Epoxy \\
\hline ETCH123487 & 9 - Impact & Sandblasted & Aremco 517 Epoxy \\
\hline ETCH123489 & 8 - Blow & Sandblasted & Aremco 517 Epoxy \\
\hline ETCH123490 & 8 - Blow & Free & Free \\
\hline ETCH123491 & 6 - Cyclic & Free & Free \\
\hline ETCH123492 & 8 - Blow & Polished & Weld \\
\hline ETCH123493 & 3 - QUV (UVB) & Polished & Aremco 517 Epoxy \\
\hline
\end{tabular}


Table B-2. 87 chemically etched stainless-steel barcode samples and associated environmental tests, continued.

\begin{tabular}{llll}
\hline Sample ID & Environmental Test & Coupon Finish & Attachment Method \\
\hline ETCH123494 & 6 - Cyclic & Sandblasted & Aremco 517 Epoxy \\
ETCH123495 & 7 - Corrosion (Fog) & Free & Free \\
ETCH123496 & 8- Blow & Free & Free \\
ETCH123497 & 3- QUV (UVB) & Sandblasted & Aremco 517 Epoxy \\
ETCH123498 & 5- Temperature & Sandblasted & Aremco 517 Epoxy \\
ETCH123499 & 5- Temperature & Sandblasted & Aremco 517 Epoxy \\
\hline
\end{tabular}


Table B-3. 92 laser-marked stainless-steel barcode samples and associated environmental tests.

\begin{tabular}{|c|c|c|c|}
\hline Sample ID & Environmental Test & Coupon Finish & Attachment Method \\
\hline YAGL123400 & 9 - Impact & Sandblasted & Aremco 517 Epoxy \\
\hline YAGL123401 & 8 - Blow & Free & Free \\
\hline YAGL123402 & 5 - Temperature & Free & Free \\
\hline YAGL123403 & 7 - Corrosion (Fog) & Free & Free \\
\hline YAGL123405 & 8 - Blow & Sandblasted & Aremco 517 Epoxy \\
\hline YAGL123406 & 10 - Wash & Free & Free \\
\hline YAGL123407 & 8 - Blow & Sandblasted & Aremco 517 Epoxy \\
\hline YAGL123408 & 7 - Corrosion (Fog) & Sandblasted & Aremco 517 Epoxy \\
\hline YAGL123409 & 5 - Temperature & Polished & Weld \\
\hline YAGL123410 & 9 - Impact & Sandblasted & Aremco 517 Epoxy \\
\hline YAGL123411 & 9 - Impact & Free & Free \\
\hline YAGL123412 & 4 - QUV \& Q-FOG & Polished & Weld \\
\hline YAGL123414 & 8 - Blow & Polished & Weld \\
\hline YAGL123415 & 6 - Cyclic & Sandblasted & Aremco 517 Epoxy \\
\hline YAGL123416 & 1 - Q-SUN & Sandblasted & Aremco 517 Epoxy \\
\hline YAGL123417 & $10-$ Wash & Free & Free \\
\hline YAGL123418 & 5 - Temperature & Free & Free \\
\hline YAGL123419 & 9 - Impact & Free & Free \\
\hline YAGL123420 & 8 - Blow & Sandblasted & Aremco 517 Epoxy \\
\hline YAGL123422 & 9 - Impact & Sandblasted & Aremco 517 Epoxy \\
\hline YAGL123423 & $10-$ Wash & Sandblasted & Aremco 517 Epoxy \\
\hline YAGL123424 & 5 - Temperature & Polished & Aremco 517 Epoxy \\
\hline YAGL123425 & 5 - Temperature & Sandblasted & Aremco 517 Epoxy \\
\hline YAGL123426 & 8 - Blow & Sandblasted & Aremco 517 Epoxy \\
\hline YAGL123427 & 7 - Corrosion (Fog) & Free & Free \\
\hline YAGL123428 & 10 - Wash & Free & Free \\
\hline YAGL123429 & $10-$ Wash & Sandblasted & Aremco 517 Epoxy \\
\hline YAGL123430 & 6 - Cyclic & Free & Free \\
\hline YAGL123432 & 5 - Temperature & Sandblasted & Aremco 517 Epoxy \\
\hline YAGL123433 & 8 - Blow & Sandblasted & Aremco 517 Epoxy \\
\hline YAGL123434 & 5 - Temperature & Free & Free \\
\hline YAGL123435 & 9 - Impact & Polished & Weld \\
\hline YAGL123437 & 3 - QUV (UVB) & Polished & Weld \\
\hline YAGL123439 & 7 - Corrosion (Fog) & Free & Free \\
\hline YAGL123440 & 3 - QUV (UVB) & Polished & Aremco 517 Epoxy \\
\hline YAGL123441 & 9 - Impact & Free & Free \\
\hline YAGL123442 & 8 - Blow & Free & Free \\
\hline YAGL123443 & 10 - Wash & Free & Free \\
\hline YAGL123444 & 8 - Blow & Free & Free \\
\hline YAGL123445 & 9 - Impact & Sandblasted & Aremco 517 Epoxy \\
\hline YAGL123446 & 9 - Impact & Polished & Weld \\
\hline
\end{tabular}


Table B-3. 92 laser-marked stainless-steel barcode samples and associated environmental tests, continued.

\begin{tabular}{|c|c|c|c|}
\hline Sample ID & Environmental Test & Coupon Finish & Attachment Method \\
\hline YAGL123447 & 7 - Corrosion (Fog) & Sandblasted & Aremco 517 Epoxy \\
\hline YAGL123448 & 7 - Corrosion (Fog) & Sandblasted & Aremco 517 Epoxy \\
\hline YAGL123449 & $10-$ Wash & Free & Free \\
\hline YAGL123450 & 5 - Temperature & Free & Free \\
\hline YAGL123451 & $10-$ Wash & Polished & Weld \\
\hline YAGL123452 & 8 - Blow & Free & Free \\
\hline YAGL123453 & 6 - Cyclic & Polished & Weld \\
\hline YAGL123455 & 7 - Corrosion (Fog) & Free & Free \\
\hline YAGL123456 & 7 - Corrosion (Fog) & Free & Free \\
\hline YAGL123457 & 1 - Q-SUN & Polished & Weld \\
\hline YAGL123458 & 2 - QUV (UVA) & Polished & Weld \\
\hline YAGL123459 & 2 - QUV (UVA) & Polished & Aremco 517 Epoxy \\
\hline YAGL123460 & 2 - QUV (UVA) & Free & Free \\
\hline YAGL123461 & 4 - QUV \& Q-FOG & Free & Free \\
\hline YAGL123462 & 4 - QUV \& Q-FOG & Polished & Aremco 517 Epoxy \\
\hline YAGL123463 & 5 - Temperature & Free & Free \\
\hline YAGL123464 & 5 - Temperature & Sandblasted & Aremco 517 Epoxy \\
\hline YAGL123465 & 8 - Blow & Free & Free \\
\hline YAGL123466 & 10 - Wash & Free & Free \\
\hline YAGL123467 & 6 - Cyclic & Free & Free \\
\hline YAGL123468 & 6 - Cyclic & Free & Free \\
\hline YAGL123469 & 9 - Impact & Free & Free \\
\hline YAGL123470 & 6 - Cyclic & Sandblasted & Aremco 517 Epoxy \\
\hline YAGL123471 & $10-$ Wash & Sandblasted & Aremco 517 Epoxy \\
\hline YAGL123472 & 7 - Corrosion (Fog) & Free & Free \\
\hline YAGL123473 & 3 - QUV (UVB) & Sandblasted & Aremco 517 Epoxy \\
\hline YAGL123474 & 6 - Cyclic & Sandblasted & Aremco 517 Epoxy \\
\hline YAGL123475 & 5 - Temperature & Sandblasted & Aremco 517 Epoxy \\
\hline YAGL123476 & 7 - Corrosion (Fog) & Polished & Weld \\
\hline YAGL123477 & 6 - Cyclic & Polished & Aremco 517 Epoxy \\
\hline YAGL123478 & 6 - Cyclic & Free & Free \\
\hline YAGL123479 & 9 - Impact & Free & Free \\
\hline YAGL123480 & 5 - Temperature & Sandblasted & Aremco 517 Epoxy \\
\hline YAGL123481 & 7 - Corrosion (Fog) & Polished & Weld \\
\hline YAGL123482 & 6 - Cyclic & Free & Free \\
\hline YAGL123483 & 9 - Impact & Free & Free \\
\hline YAGL123484 & 8 - Blow & Polished & Weld \\
\hline YAGL123485 & $10-$ Wash & Sandblasted & Aremco 517 Epoxy \\
\hline YAGL123486 & 7 - Corrosion (Fog) & Sandblasted & Aremco 517 Epoxy \\
\hline YAGL123488 & 2 - QUV (UVA) & Sandblasted & Aremco 517 Epoxy \\
\hline YAGL123489 & 6 - Cyclic & Sandblasted & Aremco 517 Epoxy \\
\hline
\end{tabular}


Table B-3. 92 laser-marked stainless-steel barcode samples and associated environmental tests, continued.

\begin{tabular}{llll}
\hline Sample ID & Environmental Test & Coupon Finish & Attachment Method \\
\hline YAGL123490 & 3- QUV (UVB) & Free & Free \\
YAGL123491 & 6- Cyclic & Sandblasted & Aremco 517 Epoxy \\
YAGL123492 & 8- Blow & Free & Free \\
YAGL123493 & 1 - Q-SUN & Polished & Aremco 517 Epoxy \\
YAGL123494 & 10- Wash & Sandblasted & Aremco 517 Epoxy \\
YAGL123495 & 10- Wash & Polished & Weld \\
YAGL123496 & 7- Corrosion (Fog) & Sandblasted & Aremco 517 Epoxy \\
YAGL123497 & 6- Cyclic & Polished & Weld \\
YAGL123498 & 6- Cyclic & Free & Free \\
YAGL123499 & 4- QUV \& Q-FOG & Sandblasted & Aremco 517 Epoxy \\
\hline
\end{tabular}


Table B-4. 48 Rebo premium vinyl labels and associated environmental tests.

\begin{tabular}{|c|c|c|c|}
\hline Sample ID & Environmental Test & Coupon Finish & Attachment Method \\
\hline REBO123402 & $6-$ Cyclic & Polished & Self-Adhered \\
\hline REBO123408 & 1 - Q-SUN & Sandblasted & Self-Adhered \\
\hline REBO123411 & $10-$ Wash & Sandblasted & Self-Adhered \\
\hline REBO123412 & 5 - Temperature & Sandblasted & Self-Adhered \\
\hline REBO123413 & 7 - Corrosion (Fog) & Sandblasted & Self-Adhered \\
\hline REBO123415 & $6-$ Cyclic & Sandblasted & Self-Adhered \\
\hline REBO123416 & 6 - Cyclic & Sandblasted & Self-Adhered \\
\hline REBO123418 & $6-$ Cyclic & Sandblasted & Self-Adhered \\
\hline REBO123420 & 9 - Impact & Sandblasted & Self-Adhered \\
\hline REBO123421 & 1 - Q-SUN & Polished & Self-Adhered \\
\hline REBO123424 & 2 - QUV (UVA) & Polished & Self-Adhered \\
\hline REBO123425 & 7 - Corrosion (Fog) & Sandblasted & Self-Adhered \\
\hline REBO123427 & 4 - QUV \& Q-FOG & Polished & Self-Adhered \\
\hline REBO123428 & 7 - Corrosion (Fog) & Sandblasted & Self-Adhered \\
\hline REBO123429 & 2 - QUV (UVA) & Sandblasted & Self-Adhered \\
\hline REBO123430 & 8 - Blow & Sandblasted & Self-Adhered \\
\hline REBO123433 & 8 - Blow & Sandblasted & Self-Adhered \\
\hline REBO123436 & 10 - Wash & Sandblasted & Self-Adhered \\
\hline REBO123439 & 10 - Wash & Sandblasted & Self-Adhered \\
\hline REBO123441 & 6 - Cyclic & Sandblasted & Self-Adhered \\
\hline REBO123442 & 4 - QUV \& Q-FOG & Sandblasted & Self-Adhered \\
\hline REBO123444 & $10-$ Wash & Sandblasted & Self-Adhered \\
\hline REBO123448 & 6 - Cyclic & Sandblasted & Self-Adhered \\
\hline REBO123450 & 9 - Impact & Sandblasted & Self-Adhered \\
\hline REBO123451 & 3 - QUV (UVB) & Sandblasted & Self-Adhered \\
\hline REBO123452 & 3 - QUV (UVB) & Sandblasted & Self-Adhered \\
\hline REBO123453 & 7 - Corrosion (Fog) & Sandblasted & Self-Adhered \\
\hline REBO123454 & 9 - Impact & Sandblasted & Self-Adhered \\
\hline REBO123455 & 1 - Q-SUN & Sandblasted & Self-Adhered \\
\hline REBO123456 & 4 - QUV \& Q-FOG & Sandblasted & Self-Adhered \\
\hline REBO123457 & 5 - Temperature & Sandblasted & Self-Adhered \\
\hline REBO123462 & 9 - Impact & Sandblasted & Self-Adhered \\
\hline REBO123463 & 5 - Temperature & Sandblasted & Self-Adhered \\
\hline REBO123466 & 2 - QUV (UVA) & Sandblasted & Self-Adhered \\
\hline REBO123467 & 5 - Temperature & Sandblasted & Self-Adhered \\
\hline REBO123468 & 9 - Impact & Sandblasted & Self-Adhered \\
\hline REBO123470 & 3 - QUV (UVB) & Polished & Self-Adhered \\
\hline REBO123471 & 8 - Blow & Sandblasted & Self-Adhered \\
\hline REBO123474 & 10 - Wash & Sandblasted & Self-Adhered \\
\hline REBO123476 & 9 - Impact & Sandblasted & Self-Adhered \\
\hline REBO123477 & 5 - Temperature & Sandblasted & Self-Adhered \\
\hline
\end{tabular}


Table B-4. 48 Rebo premium vinyl labels and associated environmental tests, continued.

\begin{tabular}{llll}
\hline \multicolumn{1}{c}{ Sample ID } & \multicolumn{1}{c}{ Environmental Test } & Coupon Finish & Attachment Method \\
\hline REBO123479 & 8 - Blow & Sandblasted & Self-Adhered \\
REBO123482 & 8 - Blow & Sandblasted & Self-Adhered \\
REBO123490 & 5 - Temperature & Polished & Self-Adhered \\
REBO123492 & 7 - Corrosion (Fog) & Sandblasted & Self-Adhered \\
REBO123494 & 7 - Corrosion (Fog) & Sandblasted & Self-Adhered \\
REBO123495 & 10 - Wash & Sandblasted & Self-Adhered \\
REBO123496 & 8 - Blow & Sandblasted & Self-Adhered \\
\hline
\end{tabular}


Table B-5. 48 Laser-marked Tesa tape samples and associated environmental tests.

\begin{tabular}{|c|c|c|c|}
\hline Sample ID & Environmental Test & Coupon Finish & Attachment Method \\
\hline TESA123402 & $6-$ Cyclic & Sandblasted & Self-Adhered \\
\hline TESA123406 & 3 - QUV (UVB) & Sandblasted & Self-Adhered \\
\hline TESA123407 & 9 - Impact & Sandblasted & Self-Adhered \\
\hline TESA123409 & 2 - QUV (UVA) & Sandblasted & Self-Adhered \\
\hline TESA123410 & $10-$ Wash & Sandblasted & Self-Adhered \\
\hline TESA123411 & 10 - Wash & Sandblasted & Self-Adhered \\
\hline TESA123412 & 9 - Impact & Sandblasted & Self-Adhered \\
\hline TESA123414 & 2 - QUV (UVA) & Polished & Self-Adhered \\
\hline TESA123415 & 4 - QUV \& Q-FOG & Sandblasted & Self-Adhered \\
\hline TESA123418 & 9 - Impact & Sandblasted & Self-Adhered \\
\hline TESA 123420 & 4 - QUV \& Q-FOG & Sandblasted & Self-Adhered \\
\hline TESA123421 & 5 - Temperature & Polished & Self-Adhered \\
\hline TESA123422 & 8 - Blow & Sandblasted & Self-Adhered \\
\hline TESA123423 & $10-$ Wash & Sandblasted & Self-Adhered \\
\hline TESA123425 & 2 - QUV (UVA) & Sandblasted & Self-Adhered \\
\hline TESA123427 & 8 - Blow & Sandblasted & Self-Adhered \\
\hline TESA123429 & 6 - Cyclic & Polished & Self-Adhered \\
\hline TESA 123430 & 6 - Cyclic & Sandblasted & Self-Adhered \\
\hline TESA123431 & 6 - Cyclic & Sandblasted & Self-Adhered \\
\hline TESA123433 & 7 - Corrosion (Fog) & Sandblasted & Self-Adhered \\
\hline TESA123437 & 7 - Corrosion (Fog) & Sandblasted & Self-Adhered \\
\hline TESA123438 & 5 - Temperature & Sandblasted & Self-Adhered \\
\hline TESA123441 & 3 - QUV (UVB) & Sandblasted & Self-Adhered \\
\hline TESA123442 & 9 - Impact & Sandblasted & Self-Adhered \\
\hline TESA123444 & 7 - Corrosion (Fog) & Sandblasted & Self-Adhered \\
\hline TESA123445 & 1 - Q-SUN & Polished & Self-Adhered \\
\hline TESA123449 & $10-$ Wash & Sandblasted & Self-Adhered \\
\hline TESA123454 & 1 - Q-SUN & Sandblasted & Self-Adhered \\
\hline TESA123456 & 6 - Cyclic & Sandblasted & Self-Adhered \\
\hline TESA123457 & 8 - Blow & Sandblasted & Self-Adhered \\
\hline TESA123460 & 5 - Temperature & Sandblasted & Self-Adhered \\
\hline TESA123462 & 5 - Temperature & Sandblasted & Self-Adhered \\
\hline TESA123463 & 5 - Temperature & Sandblasted & Self-Adhered \\
\hline TESA123465 & 1 - Q-SUN & Sandblasted & Self-Adhered \\
\hline TESA123468 & 8 - Blow & Sandblasted & Self-Adhered \\
\hline TESA123469 & 8 - Blow & Sandblasted & Self-Adhered \\
\hline TESA 123470 & 4 - QUV \& Q-FOG & Polished & Self-Adhered \\
\hline TESA 123472 & 7 - Corrosion (Fog) & Sandblasted & Self-Adhered \\
\hline TESA123473 & 5 - Temperature & Sandblasted & Self-Adhered \\
\hline TESA123475 & 8 - Blow & Sandblasted & Self-Adhered \\
\hline TESA123478 & 3 - QUV (UVB) & Polished & Self-Adhered \\
\hline TESA123483 & 9 - Impact & Sandblasted & Self-Adhered \\
\hline
\end{tabular}


Table B-5. 48 Laser-marked Tesa tape samples and associated environmental tests, continued.

\begin{tabular}{llll}
\hline Sample ID & Environmental Test & Coupon Finish & Attachment Method \\
\hline TESA123486 & 7 - Corrosion (Fog) & Sandblasted & Self-Adhered \\
TESA123488 & 10 - Wash & Sandblasted & Self-Adhered \\
TESA123491 & 6 - Cyclic & Sandblasted & Self-Adhered \\
TESA123493 & 10 - Wash & Sandblasted & Self-Adhered \\
TESA123494 & 9 - Impact & Sandblasted & Self-Adhered \\
TESA123498 & 7 - Corrosion (Fog) & Sandblasted & Self-Adhered \\
\hline
\end{tabular}


Table B-6. 34 Zebra Z-Endure 4000T labels and associated environmental tests.

\begin{tabular}{|c|c|c|c|}
\hline Sample ID & Environmental Test & Coupon Finish & Attachment Method \\
\hline ZEND451007 & 7 - Corrosion (Fog) & Sandblasted & Self-Adhered \\
\hline ZEND451008 & 8 - Blow & Sandblasted & Self-Adhered \\
\hline ZEND451201 & 4 - QUV \& Q-FOG & Sandblasted & Self-Adhered \\
\hline ZEND451202 & 4 - QUV \& Q-FOG & Polished & Self-Adhered \\
\hline ZEND451203 & 1 - Q-SUN & Sandblasted & Self-Adhered \\
\hline ZEND451204 & 2 - QUV (UVA) & Sandblasted & Self-Adhered \\
\hline ZEND451205 & 6 - Cyclic & Sandblasted & Self-Adhered \\
\hline ZEND451206 & $10-$ Wash & Sandblasted & Self-Adhered \\
\hline ZEND451207 & 7 - Corrosion (Fog) & Sandblasted & Self-Adhered \\
\hline ZEND451208 & 8 - Blow & Sandblasted & Self-Adhered \\
\hline ZEND451209 & 6 - Cyclic & Sandblasted & Self-Adhered \\
\hline ZEND451210 & 9 - Impact & Sandblasted & Self-Adhered \\
\hline ZEND451211 & 2 - QUV (UVA) & Sandblasted & Self-Adhered \\
\hline ZEND451212 & 5 - Temperature & Sandblasted & Self-Adhered \\
\hline ZEND451213 & 1 - Q-SUN & Sandblasted & Self-Adhered \\
\hline ZEND451214 & 3 - QUV (UVB) & Polished & Self-Adhered \\
\hline ZEND451215 & 6 - Cyclic & Polished & Self-Adhered \\
\hline ZEND451216 & 9 - Impact & Sandblasted & Self-Adhered \\
\hline ZEND451217 & 5 - Temperature & Sandblasted & Self-Adhered \\
\hline ZEND451218 & 9 - Impact & Sandblasted & Self-Adhered \\
\hline ZEND451219 & 3 - QUV (UVB) & Sandblasted & Self-Adhered \\
\hline ZEND451220 & 4 - QUV \& Q-FOG & Sandblasted & Self-Adhered \\
\hline ZEND451221 & 3 - QUV (UVB) & Sandblasted & Self-Adhered \\
\hline ZEND451222 & 5 - Temperature & Sandblasted & Self-Adhered \\
\hline ZEND451223 & 10 - Wash & Sandblasted & Self-Adhered \\
\hline ZEND451224 & 10 - Wash & Sandblasted & Self-Adhered \\
\hline ZEND451225 & 5 - Temperature & Sandblasted & Self-Adhered \\
\hline ZEND451226 & 5 - Temperature & Polished & Self-Adhered \\
\hline ZEND451227 & 5 - Temperature & Sandblasted & Self-Adhered \\
\hline ZEND451228 & 1 - Q-SUN & Polished & Self-Adhered \\
\hline ZEND451230 & 2 - QUV (UVA) & Polished & Self-Adhered \\
\hline ZEND451231 & 6 - Cyclic & Sandblasted & Self-Adhered \\
\hline ZEND451232 & 7 - Corrosion (Fog) & Sandblasted & Self-Adhered \\
\hline ZEND451233 & 8 - Blow & Sandblasted & Self-Adhered \\
\hline
\end{tabular}


Table B-7. 48 Zebra Z-Ultimate 3000T labels and associated environmental tests.

\begin{tabular}{|c|c|c|c|}
\hline Sample ID & Environmental Test & Coupon Finish & Attachment Method \\
\hline ZULT351003 & 7 - Corrosion (Fog) & Sandblasted & Self-Adhered \\
\hline ZULT351006 & 5 - Temperature & Sandblasted & Self-Adhered \\
\hline ZULT351007 & 9 - Impact & Sandblasted & Self-Adhered \\
\hline ZULT351009 & 5 - Temperature & Sandblasted & Self-Adhered \\
\hline ZULT351012 & 8 - Blow & Sandblasted & Self-Adhered \\
\hline ZULT351013 & 10 - Wash & Sandblasted & Self-Adhered \\
\hline ZULT351014 & 7 - Corrosion (Fog) & Sandblasted & Self-Adhered \\
\hline ZULT351015 & $6-$ Cyclic & Sandblasted & Self-Adhered \\
\hline ZULT351016 & $10-$ Wash & Sandblasted & Self-Adhered \\
\hline ZULT351017 & 2 - QUV (UVA) & Sandblasted & Self-Adhered \\
\hline ZULT351023 & 8 - Blow & Sandblasted & Self-Adhered \\
\hline ZULT351034 & 7 - Corrosion (Fog) & Sandblasted & Self-Adhered \\
\hline ZULT351035 & 8 - Blow & Sandblasted & Self-Adhered \\
\hline ZULT351036 & 5 - Temperature & Polished & Self-Adhered \\
\hline ZULT351037 & 2 - QUV (UVA) & Sandblasted & Self-Adhered \\
\hline ZULT351038 & $6-$ Cyclic & Sandblasted & Self-Adhered \\
\hline ZULT351039 & 9 - Impact & Sandblasted & Self-Adhered \\
\hline ZULT351040 & 3 - QUV (UVB) & Sandblasted & Self-Adhered \\
\hline ZULT351041 & 8 - Blow & Sandblasted & Self-Adhered \\
\hline ZULT351042 & 4 - QUV \& Q-FOG & Polished & Self-Adhered \\
\hline ZULT351043 & $6-$ Cyclic & Sandblasted & Self-Adhered \\
\hline ZULT351047 & 1 - Q-SUN & Sandblasted & Self-Adhered \\
\hline ZULT351051 & 6 - Cyclic & Polished & Self-Adhered \\
\hline ZULT351052 & 8 - Blow & Sandblasted & Self-Adhered \\
\hline ZULT351054 & 10 - Wash & Sandblasted & Self-Adhered \\
\hline ZULT351056 & 9 - Impact & Sandblasted & Self-Adhered \\
\hline ZULT351057 & 7 - Corrosion (Fog) & Sandblasted & Self-Adhered \\
\hline ZULT351058 & 5 - Temperature & Sandblasted & Self-Adhered \\
\hline ZULT351059 & 10 - Wash & Sandblasted & Self-Adhered \\
\hline ZULT351062 & 7 - Corrosion (Fog) & Sandblasted & Self-Adhered \\
\hline ZULT351064 & 5 - Temperature & Sandblasted & Self-Adhered \\
\hline ZULT351065 & $10-$ Wash & Sandblasted & Self-Adhered \\
\hline ZULT351066 & 1 - Q-SUN & Polished & Self-Adhered \\
\hline ZULT351067 & 9 - Impact & Sandblasted & Self-Adhered \\
\hline ZULT351069 & 9 - Impact & Sandblasted & Self-Adhered \\
\hline ZULT351070 & 4 - QUV \& Q-FOG & Sandblasted & Self-Adhered \\
\hline ZULT351071 & 4 - QUV \& Q-FOG & Sandblasted & Self-Adhered \\
\hline ZULT351073 & 5 - Temperature & Sandblasted & Self-Adhered \\
\hline ZULT351076 & 8 - Blow & Sandblasted & Self-Adhered \\
\hline ZULT351080 & 2 - QUV (UVA) & Polished & Self-Adhered \\
\hline ZULT351085 & 9 - Impact & Sandblasted & Self-Adhered \\
\hline ZULT351088 & 7 - Corrosion (Fog) & Sandblasted & Self-Adhered \\
\hline
\end{tabular}


Table B-7. 48 Zebra Z-Ultimate 3000T labels and associated environmental tests, continued.

\begin{tabular}{llll}
\hline Sample ID & Environmental Test & Coupon Finish & Attachment Method \\
\hline ZULT351093 & 6 - Cyclic & Sandblasted & Self-Adhered \\
ZULT351095 & 3 - QUV (UVB) & Polished & Self-Adhered \\
ZULT351096 & 6 - Cyclic & Sandblasted & Self-Adhered \\
ZULT351097 & 3 - QUV (UVB) & Sandblasted & Self-Adhered \\
ZULT351098 & 1 - Q-SUN & Sandblasted & Self-Adhered \\
ZULT351099 & 10 - Wash & Sandblasted & Self-Adhered \\
\hline
\end{tabular}


APPENDIX C. BARCODE SAMPLES GROUPED BY ENVIRONMENTAL TESTS 



\section{APPENDIX C. BARCODE SAMPLES GROUPED BY ENVIRONMENTAL TESTS}

Tables C-1 through C-10 indicate which samples were subjected to each of the 10 environmental tests. Provided for each sample is the test position, identification number, coupon finish, and attachment method.

Table C-1. 22 barcode samples subjected to Q-SUN xenon arc lamp testing (Test 1).

\begin{tabular}{clll}
\hline Test Position & Sample ID & Coupon Finish & Attachment Method \\
\hline 1 & CERM123422 & Polished & Aremco 517 Epoxy \\
2 & CERM123446 & Polished & Weld \\
3 & ZULT351066 & Polished & Self-Adhered \\
4 & YAGL123457 & Polished & Weld \\
5 & ZEND451203 & Sandblasted & Self-Adhered \\
6 & ZULT351098 & Sandblasted & Self-Adhered \\
7 & REBO123455 & Sandblasted & Self-Adhered \\
8 & CERM123413 & Free & Free \\
9 & TESA123445 & Polished & Self-Adhered \\
10 & REBO123421 & Polished & Self-Adhered \\
11 & TESA123454 & Sandblasted & Self-Adhered \\
12 & CERM123495 & Sandblasted & Aremco 517 Epoxy \\
13 & TESA123465 & Sandblasted & Self-Adhered \\
14 & ETCH123416 & Polished & Aremco 517 Epoxy \\
15 & ETCH123480 & Polished & Weld \\
16 & YAGL123416 & Sandblasted & Aremco 517 Epoxy \\
17 & ZEND451213 & Sandblasted & Self-Adhered \\
18 & ZULT351047 & Sandblasted & Self-Adhered \\
19 & ETCH123438 & Sandblasted & Aremco 517 Epoxy \\
20 & YAGL123493 & Polished & Aremco 517 Epoxy \\
21 & ZEND451228 & Polished & Self-Adhered \\
22 & REBO123408 & Sandblasted & Self-Adhered \\
\hline
\end{tabular}


Table C-2. 24 barcode samples subjected to QUV UVA testing (Test 2).

\begin{tabular}{clll}
\hline Test Position & Sample ID & Coupon Finish & Attachment Method \\
\hline 1 & CERM123402 & Polished & Weld \\
2 & ETCH123430 & Sandblasted & Aremco 517 Epoxy \\
3 & REBO123466 & Sandblasted & Self-Adhered \\
4 & ZEND451211 & Sandblasted & Self-Adhered \\
5 & CERM123488 & Sandblasted & Aremco 517 Epoxy \\
6 & TESA123409 & Sandblasted & Self-Adhered \\
7 & ETCH123409 & Polished & Aremco 517 Epoxy \\
8 & CERM123432 & Free & Free \\
9 & TESA123425 & Sandblasted & Self-Adhered \\
10 & REBO123424 & Polished & Self-Adhered \\
11 & YAGL123459 & Polished & Aremco 517 Epoxy \\
12 & TESA123414 & Polished & Self-Adhered \\
13 & ZULT351037 & Sandblasted & Self-Adhered \\
14 & YAGL123460 & Free & Free \\
15 & ETCH123473 & Polished & Weld \\
16 & ZULT351080 & Polished & Self-Adhered \\
17 & CERM123460 & Polished & Aremco 517 Epoxy \\
18 & ZEND451204 & Sandblasted & Self-Adhered \\
19 & YAGL123488 & Sandblasted & Aremco 517 Epoxy \\
20 & ZULT351017 & Sandblasted & Self-Adhered \\
21 & ZEND451230 & Polished & Self-Adhered \\
22 & REBO123429 & Sandblasted & Self-Adhered \\
23 & YAGL123458 & Polished & Weld \\
24 & ETCH123422 & Free & Free \\
\hline & & &
\end{tabular}


Table C-3. 24 barcode samples subjected to QUV UVB testing (Test 3).

\begin{tabular}{clll}
\hline Test Position & Sample ID & Coupon Finish & Attachment Method \\
\hline 1 & REBO123452 & Sandblasted & Self-Adhered \\
2 & CERM123436 & Sandblasted & Aremco 517 Epoxy \\
3 & ZEND451219 & Sandblasted & Self-Adhered \\
4 & CERM123465 & Polished & Weld \\
5 & ZULT351097 & Sandblasted & Self-Adhered \\
6 & YAGL123437 & Polished & Weld \\
7 & REBO123470 & Polished & Self-Adhered \\
8 & ETCH123497 & Sandblasted & Aremco 517 Epoxy \\
9 & ZEND451214 & Polished & Self-Adhered \\
10 & REBO123451 & Sandblasted & Self-Adhered \\
11 & YAGL123473 & Sandblasted & Aremco 517 Epoxy \\
12 & ETCH123493 & Polished & Aremco 517 Epoxy \\
13 & CERM123438 & Free & Free \\
14 & TESA123441 & Sandblasted & Self-Adhered \\
15 & YAGL123440 & Polished & Aremco 517 Epoxy \\
16 & ZEND451221 & Sandblasted & Self-Adhered \\
17 & ETCH123437 & Polished & Self-Adhered \\
18 & CERM123499 & Polished & Aremco 517 Epoxy \\
19 & ZULT351095 & Polished & Self-Adhered \\
20 & ZULT351040 & Sandblasted & Self-Adhered \\
21 & TESA123406 & Sandblasted & Self-Adhered \\
22 & YAGL123490 & Free & Free \\
23 & TESA123478 & Polished & Self-Adhered \\
24 & ETCH123407 & Free & Free \\
\hline & & &
\end{tabular}


Table C-4. 24 barcode samples subjected to QUV and Q-FOG testing (Test 4).

\begin{tabular}{clll}
\hline Test Position & Sample ID & Coupon Finish & Attachment Method \\
\hline 1 & ZULT351070 & Sandblasted & Self-Adhered \\
2 & YAGL123499 & Sandblasted & Aremco 517 Epoxy \\
3 & ZEND451202 & Polished & Self-Adhered \\
4 & CERM123450 & Polished & Aremco 517 Epoxy \\
5 & ZEND451220 & Sandblasted & Self-Adhered \\
6 & ETCH123406 & Sandblasted & Aremco 517 Epoxy \\
7 & REBO123442 & Sandblasted & Self-Adhered \\
8 & ETCH123412 & Free & Free \\
9 & CERM123425 & Sandblasted & Aremco 517 Epoxy \\
10 & TESA123420 & Sandblasted & Self-Adhered \\
11 & ZEND451201 & Sandblasted & Self-Adhered \\
12 & ZULT351042 & Polished & Self-Adhered \\
13 & TESA123470 & Polished & Self-Adhered \\
14 & REBO123427 & Polished & Self-Adhered \\
15 & YAGL123461 & Free & Free \\
16 & YAGL123462 & Polished & Aremco 517 Epoxy \\
17 & ETCH123431 & Polished & Weld \\
18 & REBO123456 & Sandblasted & Self-Adhered \\
19 & CERM123441 & Free & Free \\
20 & ZULT351071 & Sandblasted & Self-Adhered \\
21 & TESA123415 & Sandblasted & Self-Adhered \\
22 & YAGL123412 & Polished & Weld \\
23 & ETCH123402 & Polished & Aremco 517 Epoxy \\
24 & CERM123414 & Polished & Weld \\
\hline
\end{tabular}


Table C-5. 61 barcode samples subjected to temperature testing (Test 5).

\begin{tabular}{|c|c|c|c|}
\hline Test Position & Sample ID & Coupon Finish & Attachment Method \\
\hline 1 & ZEND451226 & Polished & Self-Adhered \\
\hline 2 & ETCH123454 & Sandblasted & Aremco 517 Epoxy \\
\hline 3 & ETCH123415 & Free & Free \\
\hline 4 & TESA123421 & Polished & Self-Adhered \\
\hline 5 & YAGL123480 & Sandblasted & Aremco 517 Epoxy \\
\hline 6 & CERM123478 & Polished & Weld \\
\hline 7 & REBO123463 & Sandblasted & Self-Adhered \\
\hline 8 & ZULT351009 & Sandblasted & Self-Adhered \\
\hline 9 & YAGL123402 & Free & Free \\
\hline 10 & YAGL123418 & Free & Free \\
\hline 11 & ZULT351073 & Sandblasted & Self-Adhered \\
\hline 12 & ETCH123481 & Free & Free \\
\hline 13 & ETCH123477 & Polished & Weld \\
\hline 14 & CERM123444 & Polished & Aremco 517 Epoxy \\
\hline 15 & ZULT351058 & Sandblasted & Self-Adhered \\
\hline 16 & YAGL123463 & Free & Free \\
\hline 17 & CERM123426 & Free & Free \\
\hline 18 & REBO123490 & Polished & Self-Adhered \\
\hline 19 & REBO123467 & Sandblasted & Self-Adhered \\
\hline 20 & ETCH123405 & Free & Free \\
\hline 21 & CERM123468 & Free & Free \\
\hline 22 & TESA123473 & Sandblasted & Self-Adhered \\
\hline 23 & TESA123460 & Sandblasted & Self-Adhered \\
\hline 24 & CERM123481 & Polished & Aremco 517 Epoxy \\
\hline 25 & REBO123457 & Sandblasted & Self-Adhered \\
\hline 26 & YAGL123432 & Sandblasted & Aremco 517 Epoxy \\
\hline 27 & TESA123438 & Sandblasted & Self-Adhered \\
\hline 28 & YAGL123434 & Free & Free \\
\hline 29 & ZULT351064 & Sandblasted & Self-Adhered \\
\hline 30 & YAGL123425 & Sandblasted & Aremco 517 Epoxy \\
\hline 31 & ZEND451222 & Sandblasted & Self-Adhered \\
\hline 32 & ETCH123483 & Sandblasted & Aremco 517 Epoxy \\
\hline 33 & ETCH123485 & Polished & Aremco 517 Epoxy \\
\hline 34 & REBO123477 & Sandblasted & Self-Adhered \\
\hline 35 & YAGL123450 & Free & Free \\
\hline 36 & ZEND451227 & Sandblasted & Self-Adhered \\
\hline 37 & CERM123421 & Sandblasted & Aremco 517 Epoxy \\
\hline 38 & CERM123498 & Sandblasted & Aremco 517 Epoxy \\
\hline 39 & CERM123417 & Free & Free \\
\hline 40 & REBO123412 & Sandblasted & Self-Adhered \\
\hline 41 & CERM123434 & Sandblasted & Aremco 517 Epoxy \\
\hline 42 & TESA123462 & Sandblasted & Self-Adhered \\
\hline
\end{tabular}


Table C-5. 61 barcode samples subjected to temperature testing (Test 5), continued.

\begin{tabular}{clll}
\hline Test Position & Sample ID & Coupon Finish & Attachment Method \\
\hline 43 & CERM123404 & Sandblasted & Aremco 517 Epoxy \\
44 & ZULT351036 & Polished & Self-Adhered \\
45 & CERM123484 & Free & Free \\
46 & ETCH123472 & Free & Free \\
47 & ZULT351006 & Sandblasted & Self-Adhered \\
48 & CERM123400 & Free & Free \\
49 & ETCH123426 & Sandblasted & Aremco 517 Epoxy \\
50 & ETCH123498 & Sandblasted & Aremco 517 Epoxy \\
51 & ZEND451212 & Sandblasted & Self-Adhered \\
52 & ETCH123499 & Sandblasted & Aremco 517 Epoxy \\
53 & YAGL123424 & Polished & Aremco 517 Epoxy \\
54 & ZEND451225 & Sandblasted & Self-Adhered \\
55 & ETCH123446 & Free & Free \\
56 & ZEND451217 & Sandblasted & Self-Adhered \\
57 & TESA123463 & Sandblasted & Self-Adhered \\
58 & YAGL123409 & Polished & Weld \\
59 & CERM123490 & Sandblasted & Aremco 517 Epoxy \\
60 & YAGL123475 & Sandblasted & Aremco 517 Epoxy \\
61 & YAGL123464 & Sandblasted & Aremco 517 Epoxy \\
\hline
\end{tabular}


Table C-6. 63 barcode samples subjected to cyclic corrosion testing (Test 6).

\begin{tabular}{|c|c|c|c|}
\hline Test Position & Sample ID & Coupon Finish & Attachment Method \\
\hline 1 & ETCH123434 & Sandblasted & Aremco 517 Epoxy \\
\hline 2 & CERM123459 & Free & Free \\
\hline 3 & ZULT351093 & Sandblasted & Self-Adhered \\
\hline 4 & TESA123429 & Polished & Self-Adhered \\
\hline 5 & REBO123441 & Sandblasted & Self-Adhered \\
\hline 6 & CERM123411 & Sandblasted & Aremco 517 Epoxy \\
\hline 7 & ETCH123478 & Polished & Weld \\
\hline 8 & TESA123456 & Sandblasted & Self-Adhered \\
\hline 9 & YAGL123478 & Free & Free \\
\hline 10 & YAGL123453 & Polished & Weld \\
\hline 11 & ZULT351015 & Sandblasted & Self-Adhered \\
\hline 12 & YAGL123468 & Free & Free \\
\hline 13 & CERM123496 & Free & Free \\
\hline 14 & YAGL123430 & Free & Free \\
\hline 15 & ZULT351043 & Sandblasted & Self-Adhered \\
\hline 16 & YAGL123497 & Polished & Weld \\
\hline 17 & ETCH123469 & Sandblasted & Aremco 517 Epoxy \\
\hline 18 & YAGL123470 & Sandblasted & Aremco 517 Epoxy \\
\hline 19 & ZULT351051 & Polished & Self-Adhered \\
\hline 20 & REBO123448 & Sandblasted & Self-Adhered \\
\hline 21 & YAGL123491 & Sandblasted & Aremco 517 Epoxy \\
\hline 22 & ETCH123436 & Polished & Weld \\
\hline 23 & CERM123405 & Free & Free \\
\hline 24 & TESA123402 & Sandblasted & Self-Adhered \\
\hline 25 & CERM123452 & Polished & Weld \\
\hline 26 & YAGL123474 & Sandblasted & Aremco 517 Epoxy \\
\hline 27 & ETCH123494 & Sandblasted & Aremco 517 Epoxy \\
\hline 28 & YAGL123477 & Polished & Aremco 517 Epoxy \\
\hline 29 & YAGL123467 & Free & Free \\
\hline 30 & ETCH123420 & Sandblasted & Aremco 517 Epoxy \\
\hline 31 & CERM123473 & Sandblasted & Aremco 517 Epoxy \\
\hline 32 & CERM123431 & Sandblasted & Aremco 517 Epoxy \\
\hline 33 & CERM123454 & Polished & Weld \\
\hline 34 & TESA123430 & Sandblasted & Self-Adhered \\
\hline 35 & ZEND451231 & Sandblasted & Self-Adhered \\
\hline 36 & CERM123475 & Sandblasted & Aremco 517 Epoxy \\
\hline 37 & CERM123423 & Free & Free \\
\hline 38 & YAGL123482 & Free & Free \\
\hline 39 & ZEND451205 & Sandblasted & Self-Adhered \\
\hline 40 & ZULT351038 & Sandblasted & Self-Adhered \\
\hline 41 & ETCH123448 & Polished & Aremco 517 Epoxy \\
\hline 42 & ETCH123464 & Free & Free \\
\hline
\end{tabular}

C-9 
Table C-6. 63 barcode samples subjected to cyclic corrosion testing (Test 6), continued.

\begin{tabular}{crll}
\hline Test Position & Sample ID & Coupon Finish & Attachment Method \\
\hline 43 & CERM123448 & Sandblasted & Aremco 517 Epoxy \\
44 & ETCH123414 & Free & Free \\
45 & REBO123402 & Polished & Self-Adhered \\
46 & REBO123416 & Sandblasted & Self-Adhered \\
47 & TESA123431 & Sandblasted & Self-Adhered \\
48 & TESA123491 & Sandblasted & Self-Adhered \\
49 & YAGL123489 & Sandblasted & Aremco 517 Epoxy \\
50 & CERM123491 & Sandblasted & Aremco 517 Epoxy \\
51 & CERM123419 & Polished & Aremco 517 Epoxy \\
52 & ETCH123450 & Free & Free \\
53 & ETCH123491 & Free & Free \\
54 & YAGL123415 & Sandblasted & Aremco 517 Epoxy \\
55 & CERM123494 & Free & Free \\
56 & REBO123418 & Sandblasted & Self-Adhered \\
57 & YAGL123498 & Free & Free \\
58 & ETCH123410 & Sandblasted & Aremco 517 Epoxy \\
59 & REBO123415 & Sandblasted & Self-Adhered \\
60 & ETCH123427 & Free & Free \\
61 & ZEND451209 & Sandblasted & Self-Adhered \\
62 & ZEND451215 & Polished & Self-Adhered \\
63 & ZULT351096 & Sandblasted & Self-Adhered \\
\hline & & &
\end{tabular}


Table C-7. 57 barcode samples subjected to corrosion testing (Test 7).

\begin{tabular}{|c|c|c|c|}
\hline Test Position & Sample ID & Coupon Finish & Attachment Method \\
\hline 1 & REBO123453 & Sandblasted & Self-Adhered \\
\hline 2 & ZEND451232 & Sandblasted & Self-Adhered \\
\hline 3 & TESA123472 & Sandblasted & Self-Adhered \\
\hline 4 & ZULT351034 & Sandblasted & Self-Adhered \\
\hline 5 & TESA123444 & Sandblasted & Self-Adhered \\
\hline 6 & ZULT351062 & Sandblasted & Self-Adhered \\
\hline 7 & CERM123462 & Free & Free \\
\hline 8 & ZULT351003 & Sandblasted & Self-Adhered \\
\hline 9 & YAGL123472 & Free & Free \\
\hline 10 & YAGL123408 & Sandblasted & Aremco 517 Epoxy \\
\hline 11 & CERM123412 & Free & Free \\
\hline 12 & YAGL123476 & Polished & Weld \\
\hline 13 & YAGL123427 & Free & Free \\
\hline 14 & YAGL123486 & Sandblasted & Aremco 517 Epoxy \\
\hline 15 & YAGL123481 & Polished & Weld \\
\hline 16 & ETCH123411 & Sandblasted & Aremco 517 Epoxy \\
\hline 17 & ETCH123458 & Polished & Weld \\
\hline 18 & TESA123437 & Sandblasted & Self-Adhered \\
\hline 19 & ZEND451207 & Sandblasted & Self-Adhered \\
\hline 20 & YAGL123456 & Free & Free \\
\hline 21 & CERM123497 & Sandblasted & Aremco 517 Epoxy \\
\hline 22 & ZULT351057 & Sandblasted & Self-Adhered \\
\hline 23 & ETCH123423 & Sandblasted & Aremco 517 Epoxy \\
\hline 24 & REBO123428 & Sandblasted & Self-Adhered \\
\hline 25 & TESA123498 & Sandblasted & Self-Adhered \\
\hline 26 & CERM123457 & Sandblasted & Aremco 517 Epoxy \\
\hline 27 & TESA123433 & Sandblasted & Self-Adhered \\
\hline 28 & ETCH123417 & Free & Free \\
\hline 29 & ZULT351088 & Sandblasted & Self-Adhered \\
\hline 30 & CERM123480 & Polished & Weld \\
\hline 31 & CERM123483 & Free & Free \\
\hline 32 & ETCH123443 & Sandblasted & Aremco 517 Epoxy \\
\hline 33 & TESA123486 & Sandblasted & Self-Adhered \\
\hline 34 & REBO123425 & Sandblasted & Self-Adhered \\
\hline 35 & YAGL123439 & Free & Free \\
\hline 36 & CERM123420 & Free & Free \\
\hline 37 & CERM123403 & Free & Free \\
\hline 38 & ETCH123495 & Free & Free \\
\hline 39 & REBO123494 & Sandblasted & Self-Adhered \\
\hline 40 & YAGL123447 & Sandblasted & Aremco 517 Epoxy \\
\hline 41 & YAGL123496 & Sandblasted & Aremco 517 Epoxy \\
\hline 42 & CERM123449 & Sandblasted & Aremco 517 Epoxy \\
\hline
\end{tabular}


Table C-7. 57 barcode samples subjected to corrosion testing (Test 7), continued.

\begin{tabular}{clll}
\hline Test Position & Sample ID & Coupon Finish & Attachment Method \\
\hline 43 & CERM123406 & Sandblasted & Aremco 517 Epoxy \\
44 & YAGL123403 & Free & Free \\
45 & ETCH123421 & Free & Free \\
46 & ZEND451007 & Sandblasted & Self-Adhered \\
47 & ETCH123476 & Free & Free \\
48 & ETCH123445 & Polished & Weld \\
49 & ETCH123484 & Free & Free \\
50 & CERM123471 & Polished & Weld \\
51 & REBO123413 & Sandblasted & Self-Adhered \\
52 & ETCH123425 & Sandblasted & Aremco 517 Epoxy \\
53 & ETCH123471 & Sandblasted & Aremco 517 Epoxy \\
54 & YAGL123455 & Free & Free \\
55 & REBO123492 & Sandblasted & Self-Adhered \\
56 & ZULT351014 & Sandblasted & Self-Adhered \\
57 & YAGL123448 & Sandblasted & Aremco 517 Epoxy \\
\hline
\end{tabular}


Table C-8. 57 barcode samples subjected to blowing sand/dust testing (Test 8).

\begin{tabular}{|c|c|c|c|}
\hline Test Position & Sample ID & Coupon Finish & Attachment Method \\
\hline 1 & ETCH123408 & Sandblasted & Aremco 517 Epoxy \\
\hline 2 & CERM123487 & Free & Free \\
\hline 3 & YAGL123426 & Sandblasted & Aremco 517 Epoxy \\
\hline 4 & YAGL123465 & Free & Free \\
\hline 5 & CERM123410 & Sandblasted & Aremco 517 Epoxy \\
\hline 6 & CERM123409 & Free & Free \\
\hline 7 & ETCH123442 & Sandblasted & Aremco 517 Epoxy \\
\hline 8 & TESA123468 & Sandblasted & Self-Adhered \\
\hline 9 & ZULT351041 & Sandblasted & Aremco 517 Epoxy \\
\hline 10 & YAGL123405 & Sandblasted & Aremco 517 Epoxy \\
\hline 11 & REBO123430 & Sandblasted & Self-Adhered \\
\hline 12 & YAGL123401 & Free & Free \\
\hline 13 & ZULT351012 & Sandblasted & Self-Adhered \\
\hline 14 & REBO123482 & Sandblasted & Self-Adhered \\
\hline 15 & ETCH123460 & Free & Free \\
\hline 16 & YAGL123442 & Free & Free \\
\hline 17 & YAGL123444 & Free & Free \\
\hline 18 & ETCH123490 & Free & Free \\
\hline 19 & CERM123476 & Polished & Weld \\
\hline 20 & REBO123471 & Sandblasted & Self-Adhered \\
\hline 21 & ETCH123496 & Free & Free \\
\hline 22 & ETCH123492 & Polished & Weld \\
\hline 23 & YAGL123452 & Free & Free \\
\hline 24 & TESA123422 & Sandblasted & Self-Adhered \\
\hline 25 & ZEND451008 & Sandblasted & Self-Adhered \\
\hline 26 & ETCH123419 & Free & Free \\
\hline 27 & CERM123447 & Free & Free \\
\hline 28 & ETCH123489 & Sandblasted & Aremco 517 Epoxy \\
\hline 29 & CERM123418 & Free & Free \\
\hline 30 & YAGL123492 & Free & Free \\
\hline 31 & ZEND451208 & Sandblasted & Self-Adhered \\
\hline 32 & TESA123457 & Sandblasted & Self-Adhered \\
\hline 33 & ZULT351035 & Sandblasted & Self-Adhered \\
\hline 34 & YAGL123433 & Sandblasted & Aremco 517 Epoxy \\
\hline 35 & ETCH123479 & Sandblasted & Aremco 517 Epoxy \\
\hline 36 & CERM123433 & Sandblasted & Aremco 517 Epoxy \\
\hline 37 & YAGL123420 & Sandblasted & Aremco 517 Epoxy \\
\hline 38 & ETCH123475 & Sandblasted & Aremco 517 Epoxy \\
\hline 39 & ZULT351052 & Sandblasted & Self-Adhered \\
\hline 40 & CERM123469 & Sandblasted & Aremco 517 Epoxy \\
\hline 41 & ZULT351023 & Sandblasted & Self-Adhered \\
\hline 42 & YAGL123414 & Polished & Weld \\
\hline
\end{tabular}

C-13 
Table C-8. 57 barcode samples subjected to blowing sand/dust testing (Test 8), continued.

\begin{tabular}{clll}
\hline Test Position & Sample ID & Coupon Finish & Attachment Method \\
\hline 43 & ETCH123457 & Free & Free \\
44 & REBO123479 & Sandblasted & Self-Adhered \\
45 & REBO123433 & Sandblasted & Self-Adhered \\
46 & YAGL123407 & Sandblasted & Aremco 517 Epoxy \\
47 & YAGL123484 & Polished & Weld \\
48 & CERM123424 & Sandblasted & Aremco 517 Epoxy \\
49 & ETCH123413 & Polished & Weld \\
50 & TESA123469 & Sandblasted & Self-Adhered \\
51 & CERM123435 & Free & Free \\
52 & ZULT351076 & Sandblasted & Self-Adhered \\
53 & ZEND451233 & Sandblasted & Self-Adhered \\
54 & REBO123496 & Sandblasted & Self-Adhered \\
55 & TESA123427 & Sandblasted & Self-Adhered \\
56 & CERM123464 & Polished & Weld \\
57 & TESA123475 & Sandblasted & Self-Adhered \\
\hline
\end{tabular}


Table C-9. 55 barcode samples subjected to impact testing (Test 9).

\begin{tabular}{|c|c|c|c|}
\hline Test Position & Sample ID & Coupon Finish & Attachment Method \\
\hline 1 & YAGL123469 & Free & Free \\
\hline 2 & CERM123407 & Free & Free \\
\hline 3 & TESA123407 & Sandblasted & Self-Adhered \\
\hline 4 & ZULT351056 & Sandblasted & Self-Adhered \\
\hline 5 & ETCH123439 & Sandblasted & Aremco 517 Epoxy \\
\hline 6 & TESA123483 & Sandblasted & Self-Adhered \\
\hline 7 & YAGL123400 & Sandblasted & Aremco 517 Epoxy \\
\hline 8 & ETCH123487 & Sandblasted & Aremco 517 Epoxy \\
\hline 9 & CERM123489 & Free & Free \\
\hline 10 & YAGL123479 & Free & Free \\
\hline 11 & ZULT351039 & Sandblasted & Self-Adhered \\
\hline 12 & ETCH123463 & Free & Free \\
\hline 13 & CERM123492 & Free & Free \\
\hline 14 & REBO123454 & Sandblasted & Self-Adhered \\
\hline 15 & ZEND451210 & Sandblasted & Self-Adhered \\
\hline 16 & REBO123450 & Sandblasted & Self-Adhered \\
\hline 17 & YAGL123445 & Sandblasted & Aremco 517 Epoxy \\
\hline 18 & CERM123479 & Sandblasted & Aremco 517 Epoxy \\
\hline 19 & CERM123440 & Sandblasted & Aremco 517 Epoxy \\
\hline 20 & TESA123412 & Sandblasted & Self-Adhered \\
\hline 21 & ETCH123429 & Free & Free \\
\hline 22 & REBO123476 & Sandblasted & Self-Adhered \\
\hline 23 & TESA123442 & Sandblasted & Self-Adhered \\
\hline 24 & TESA123494 & Sandblasted & Self-Adhered \\
\hline 25 & ETCH123466 & Polished & Weld \\
\hline 26 & TESA123418 & Sandblasted & Self-Adhered \\
\hline 27 & YAGL123435 & Polished & Weld \\
\hline 28 & YAGL123410 & Sandblasted & Aremco 517 Epoxy \\
\hline 29 & ZEND451216 & Sandblasted & Self-Adhered \\
\hline 30 & ETCH123403 & Sandblasted & Aremco 517 Epoxy \\
\hline 31 & CERM123428 & Free & Free \\
\hline 32 & REBO123462 & Sandblasted & Self-Adhered \\
\hline 33 & CERM123485 & Polished & Weld \\
\hline 34 & CERM123466 & Sandblasted & Aremco 517 Epoxy \\
\hline 35 & ZULT351069 & Sandblasted & Self-Adhered \\
\hline 36 & YAGL123446 & Polished & Weld \\
\hline 37 & YAGL123411 & Free & Free \\
\hline 38 & YAGL123419 & Free & Free \\
\hline 39 & ETCH123470 & Free & Free \\
\hline 40 & CERM123458 & Free & Free \\
\hline 41 & ZULT351085 & Sandblasted & Self-Adhered \\
\hline 42 & ZULT351067 & Sandblasted & Self-Adhered \\
\hline
\end{tabular}

C-15 
Table C-9. 55 barcode samples subjected to impact testing (Test 9), continued.

\begin{tabular}{clll}
\hline Test Position & Sample ID & Coupon Finish & Attachment Method \\
\hline 43 & REBO123420 & Sandblasted & Self-Adhered \\
44 & ZULT351007 & Sandblasted & Self-Adhered \\
45 & ETCH123401 & Free & Free \\
46 & CERM123493 & Polished & Weld \\
47 & YAGL123441 & Free & Free \\
48 & REBO123468 & Sandblasted & Self-Adhered \\
49 & ZEND451218 & Sandblasted & Self-Adhered \\
50 & ETCH123400 & Polished & Weld \\
51 & YAGL123483 & Free & Free \\
52 & ETCH123441 & Sandblasted & Aremco 517 Epoxy \\
53 & YAGL123422 & Sandblasted & Aremco 517 Epoxy \\
54 & ETCH123424 & Free & Free \\
55 & CERM123482 & Sandblasted & Aremco 517 Epoxy \\
\hline
\end{tabular}


Table C-10. 57 barcode samples subjected to high pressure and temperature water jet (Test 10).

\begin{tabular}{|c|c|c|c|}
\hline Test Position & Sample ID & Coupon Finish & Attachment Method \\
\hline 1 & ZULT351016 & Sandblasted & Self-Adhered \\
\hline 2 & REBO123474 & Sandblasted & Self-Adhered \\
\hline 3 & CERM123472 & Sandblasted & Aremco 517 Epoxy \\
\hline 4 & REBO123411 & Sandblasted & Self-Adhered \\
\hline 5 & YAGL123449 & Free & Free \\
\hline 6 & ETCH123447 & Free & Free \\
\hline 7 & ZULT351059 & Sandblasted & Self-Adhered \\
\hline 8 & REBO123439 & Sandblasted & Self-Adhered \\
\hline 9 & ZULT351099 & Sandblasted & Self-Adhered \\
\hline 10 & ZEND451223 & Sandblasted & Self-Adhered \\
\hline 11 & YAGL123428 & Free & Free \\
\hline 12 & ETCH123444 & Free & Free \\
\hline 13 & TESA123423 & Sandblasted & Self-Adhered \\
\hline 14 & CERM123427 & Free & Free \\
\hline 15 & ETCH123482 & Sandblasted & Aremco 517 Epoxy \\
\hline 16 & ETCH123461 & Free & Free \\
\hline 17 & ZULT351065 & Sandblasted & Self-Adhered \\
\hline 18 & CERM123430 & Sandblasted & Aremco 517 Epoxy \\
\hline 19 & TESA123411 & Sandblasted & Self-Adhered \\
\hline 20 & ETCH123474 & Sandblasted & Aremco 517 Epoxy \\
\hline 21 & CERM123470 & Polished & Weld \\
\hline 22 & ZEND451206 & Sandblasted & Self-Adhered \\
\hline 23 & ETCH123452 & Free & Free \\
\hline 24 & CERM123401 & Free & Free \\
\hline 25 & TESA123449 & Sandblasted & Self-Adhered \\
\hline 26 & CERM123461 & Polished & Weld \\
\hline 27 & ETCH123404 & Sandblasted & Aremco 517 Epoxy \\
\hline 28 & CERM123416 & Sandblasted & Aremco 517 Epoxy \\
\hline 29 & ZULT351013 & Sandblasted & Self-Adhered \\
\hline 30 & ETCH123451 & Free & Free \\
\hline 31 & CERM123445 & Free & Free \\
\hline 32 & ЕTCH123449 & Sandblasted & Aremco 517 Epoxy \\
\hline 33 & TESA123493 & Sandblasted & Self-Adhered \\
\hline 34 & ETCH123467 & Polished & Weld \\
\hline 35 & REBO123436 & Sandblasted & Self-Adhered \\
\hline 36 & ETCH123459 & Polished & Weld \\
\hline 37 & TESA123410 & Sandblasted & Self-Adhered \\
\hline 38 & YAGL123443 & Free & Free \\
\hline 39 & ZEND451224 & Sandblasted & Self-Adhered \\
\hline 40 & REBO123444 & Sandblasted & Self-Adhered \\
\hline 41 & ZULT351054 & Sandblasted & Self-Adhered \\
\hline 42 & YAGL123485 & Sandblasted & Aremco 517 Еpoxy \\
\hline
\end{tabular}

C-17 
Table C-10. 57 barcode samples subjected to high pressure and temperature water jet (Test 10), continued.

\begin{tabular}{clll}
\hline Test Position & Sample ID & Coupon Finish & Attachment Method \\
\hline 43 & ETCH123468 & Sandblasted & Aremco 517 Epoxy \\
44 & YAGL123417 & Free & Free \\
45 & YAGL123494 & Sandblasted & Aremco 517 Epoxy \\
46 & YAGL123451 & Polished & Weld \\
47 & YAGL123471 & Sandblasted & Aremco 517 Epoxy \\
48 & YAGL123429 & Sandblasted & Aremco 517 Epoxy \\
49 & YAGL123406 & Free & Free \\
50 & YAGL123423 & Sandblasted & Aremco 517 Epoxy \\
51 & TESA123488 & Sandblasted & Self-Adhered \\
52 & CERM123467 & Free & Free \\
53 & CERM123451 & Sandblasted & Aremco 517 Epoxy \\
54 & YAGL123466 & Free & Free \\
55 & REBO123495 & Sandblasted & Self-Adhered \\
56 & YAGL123495 & Polished & Weld \\
57 & CERM123443 & Free & Free \\
\hline
\end{tabular}

